\title{
Evaluierung der OSTA in Washington
}

\author{
und Peking
}

\section{Endbericht}

\section{September 2019}

Bankverbindung: Bank Austria Creditanstali IBAN AT69 1200000684137409 BIC BKAUATWW 


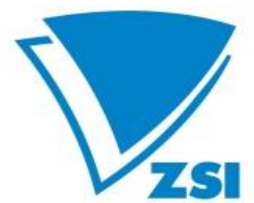

Evaluierung der OSTA in Washington und Peking

Endbericht

ZSI - Zentrum für Soziale Innovation gGmbH

Subauftragnehmer: JOANNEUM RESEARCH - POLICIES

\section{Autorinnen und Autoren:}

Dorothea Sturn, ZSI (Projektleitung)

Helmut Gassler, ZSI

Michael Ploder, JOANNEUM RESEARCH

Klaus Schuch, ZSI

Maximilian Unger, JOANNEUM RESEARCH 


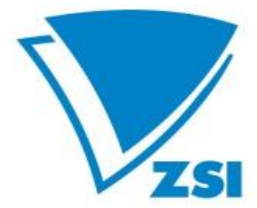

ZENTRUM FÜR SOZIALE INNOVATION

CENTRE FOR SOCIAL INNOVATION

\section{Inhalt}

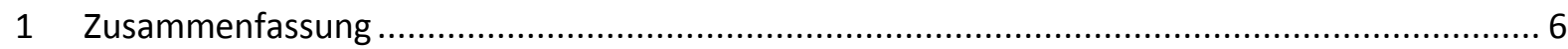

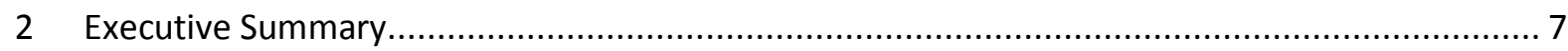

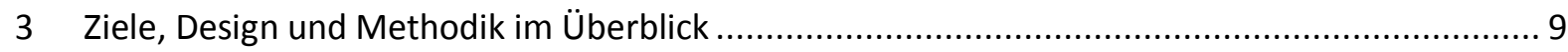

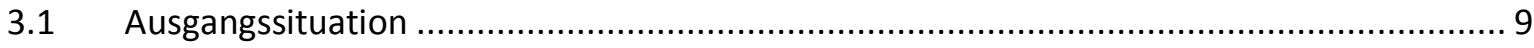

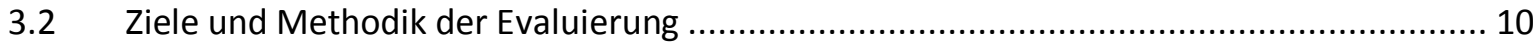

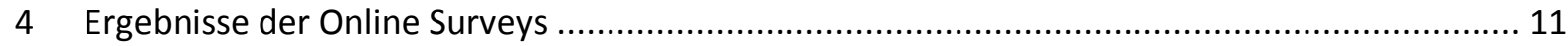

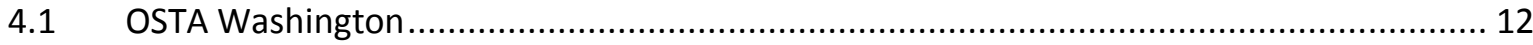

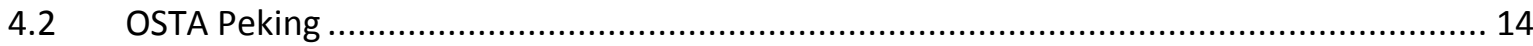

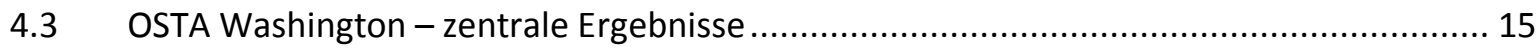

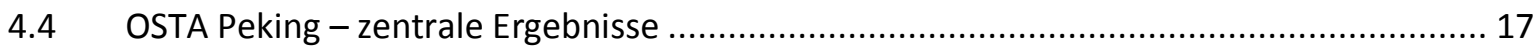

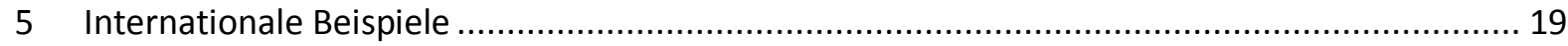

$5.1 \quad$ Schweden

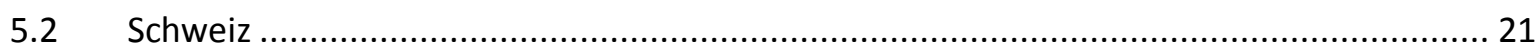

6 Wirkungsmodelle entlang einer Output - Outcome - Impact Logik .................................... 27

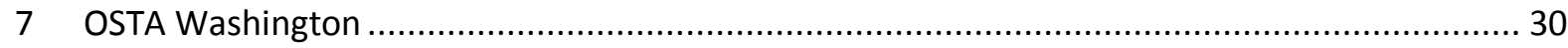

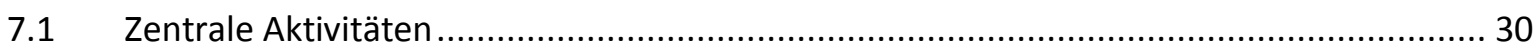

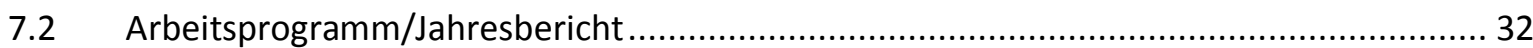

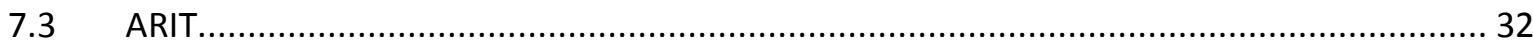

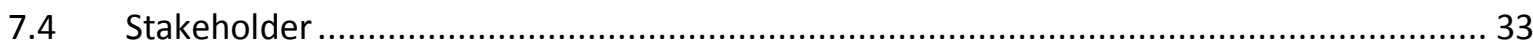

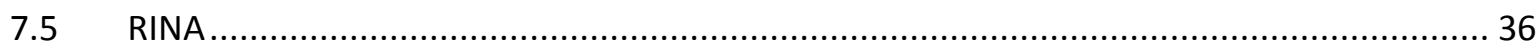

7.6 Governance vor Ort, Abstimmung mit anderen Akteuren .............................................. 40

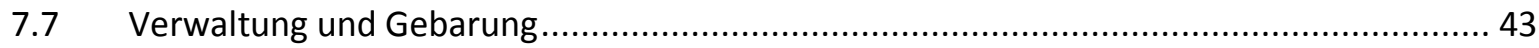

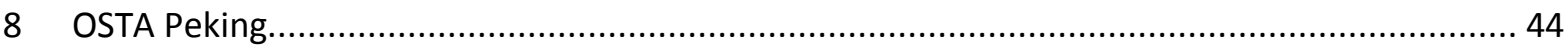




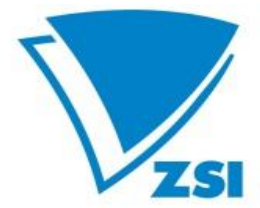

ZENTRUM FÜR SOZIALE INNOVATION

CENTRE FOR SOCIAL INNOVATION

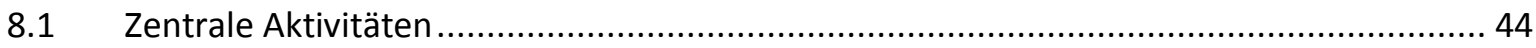

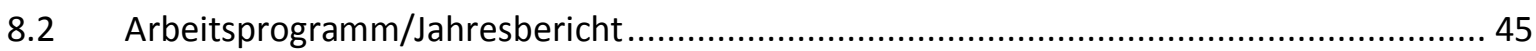

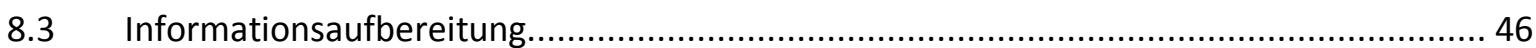

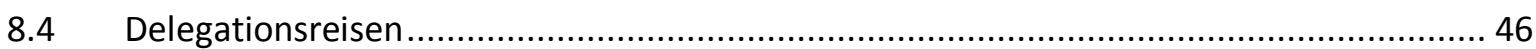

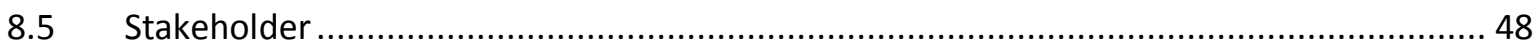

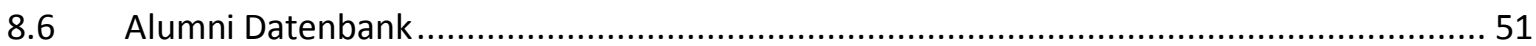

8.7 Governance vor Ort, Abstimmung mit anderen Akteuren ........................................... 52

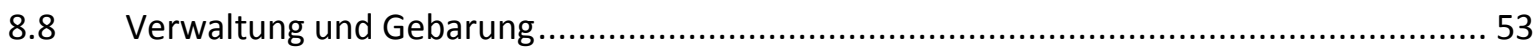

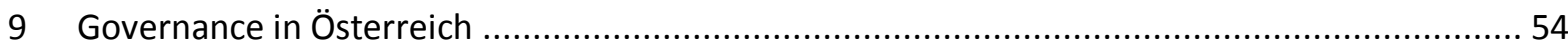

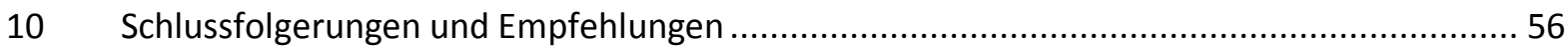

10.1 Szenario 1: kleine OSTA mit variabler Geometrie ....................................................... 58

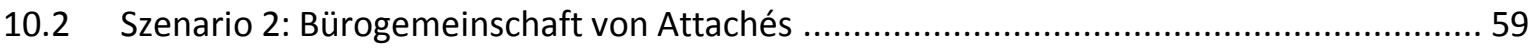

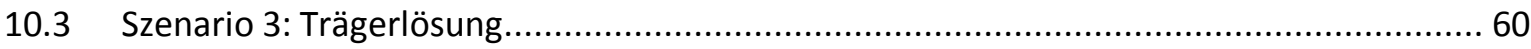

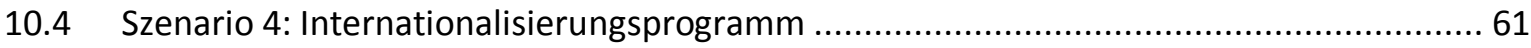

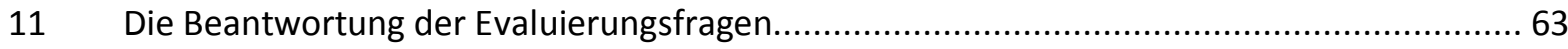

12 Anhänge

12.1 Interviewte Personen und Teilnehmende Fokusgruppen ............................................ 70

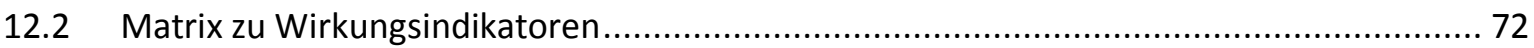

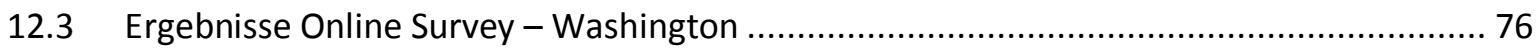

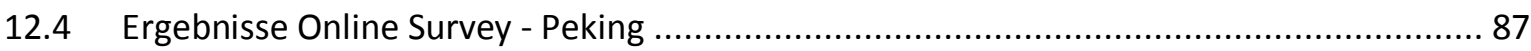




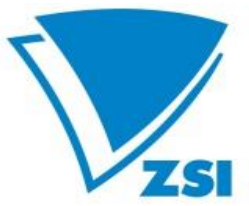

\section{Abbildungen}

Ohne die Abbildungen in den Anhängen

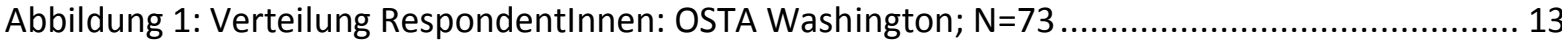

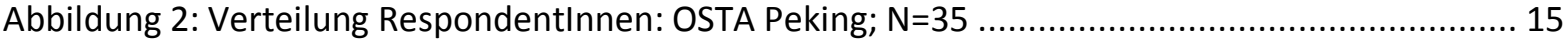

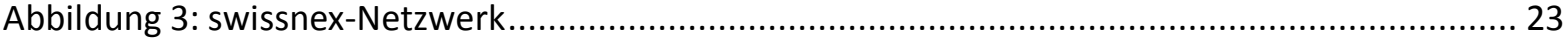

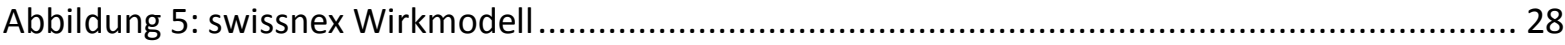

Abbildung 6: Aufgaben, die OSTA Washington künftig stärker wahrnehmen soll ........................... 34

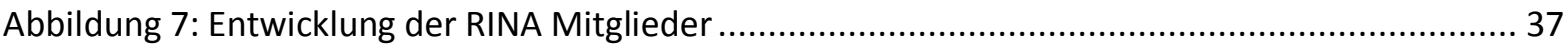

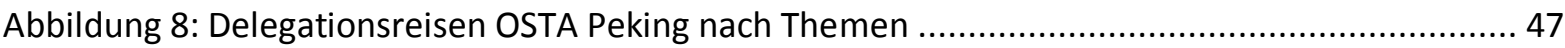

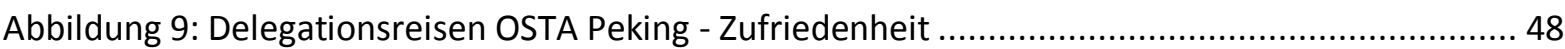

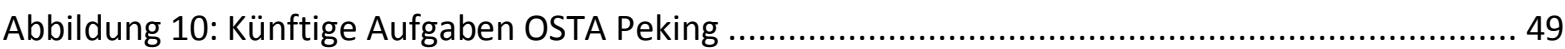

Abbildung 11: Szenario 1 - Kleine OSTA mit variabler Geometrie................................................... 58

Abbildung 12: Szenario 2 - Bürogemeinschaft von Attachés ....................................................... 59

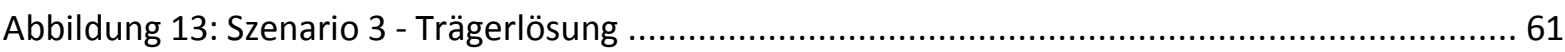

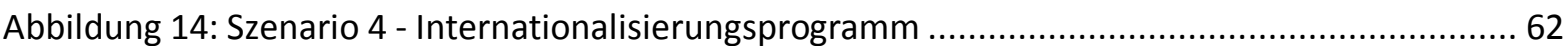




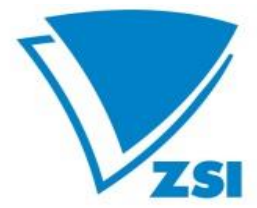

ZENTRUM FÜR SOZIALE INNOVATION

CENTRE FOR SOCIAL INNOVATION

\section{Zusammenfassung}

Die beiden „Offices of Science and Technology Austria“ (OSTA) in Washington D.C. und Peking zielen darauf ab, Österreich an führende und wichtige Forschungs-, Technologie- und Innovationsnationen (FTI-Nationen) anzudocken sowie den Wissens- und Technologietransfer auch über Europa hinaus zu unterstützen. Beide OSTA haben ihren Sitz in den österreichischen Botschaften, werden von einer Person aus dem diplomatischen Dienst geleitet und gemäß einer gemeinsamen interministeriellen Rahmenvereinbarung von vier Ressorts (Bundesministerium für Europa, Integration und Äußeres, Bundesministerium für Bildung, Wissenschaft und Forschung, Bundesministerium für Digitalisierung und Wirtschaftsstandort sowie Bundesministerium für Verkehr, Innovation und Technologie) gesteuert.

Die vorliegende Evaluierung nimmt eine Einschätzung der Aktivitäten sowie deren Wirkung - auch im Vergleich mit zwei anderen europäischen Ländern, Schweden und der Schweiz - vor, bewertet Reporting und Monitoring, schätzt Nutzen und Bedeutung ab, schlägt Empfehlungen vor und entwirft vier Zukunftsszenarien.

Es wurden ein Online-Survey eingesetzt, Interviews geführt, Berichte und Literatur recherchiert, zwei Fokusgruppen organisiert sowie je ein Besuch vor Ort durchgeführt.

Beide Büros gehen ihren Aufgaben aktiv und mit viel Engagement nach, die Arbeitsprogramme werden weitgehend umgesetzt. Sie sind mit anderen (österreichischen) Organisationen vor Ort gut vernetzt, die Mitarbeitenden agieren serviceorientiert, kompetent und qualifiziert. Auch die Personen und Organisationen, welche Leistungen der OSTA in Anspruch nehmen, sind mehrheitlich zufrieden. Schwachstellen und Probleme zeigen sich bei den folgenden Punkten:

- Die interministerielle Governance erweist sich als inflexibel, kompliziert und zeitaufwändig. Die Ressorts können den Gewinn für ihr eigenes Haus vielfach nicht approbieren.

- Bei OSTA Peking konzentriert sich sehr viel Zeit und Energie in die Organisation und Betreuung von Delegationsreisen, auch ergeben sich Überlappungen mit den Aktivitäten der AußenwirtschaftsCenters.

- Bei OSTA Washington liegt der Fokus auf der Betreuung und Beratung von Forschenden und insbesondere der Organisation des jährlichen ARIT. Vor allem das BMIT sieht angesichts dieser Orientierung seine Interessen nicht vertreten. 


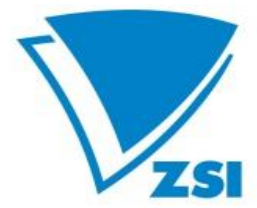

- Die Governance vor Ort kann zu Kompetenzstreitigkeiten zwischen der Vertretungsbehörde, der Leitung der OSTA und den inhaltlich verantwortlichen Ressorts führen.

- Die Leitungspersonen sind aufgrund ihres Diplomatenstatus nicht eng mit der österreichischen FTI Szene verbunden.

Aus diesen Befunden heraus empfiehlt sich in jedem Fall eine Reduktion des Aufwandes und eine Flexibilisierung der jetzigen Konstruktion. Folgende Empfehlungen formuliert die Evaluierung:

- Klare Positionierung, Definition der Ziele, der Agenda und der strategischen Orientierung

- Anschlussfähigkeit in Österreich verbessern

- Kooperation mit und Abgrenzung zu den AußenwirtschaftsCentern (AWC) definieren

- Berichtswesen und Reporting optimieren und mehrjährige Planungshorizonte einführen

- Reduktion des operativen Aufwandes auf allen Seiten

Jedes Zukunftsszenario soll so gestaltet sein, dass der Nutzen für die Kunden und Kundinnen wie auch Motivation und Engagement in den Teams zumindest bestehen bleibt, die Zufriedenheit der Ressorts jedoch gesteigert wird. Folgende Szenarien schlägt die Evaluierung vor:

- Szenario 1: kleine OSTA mit variabler Geometrie

- Szenario 2: Bürogemeinschaft von Attachés

- Szenario 3: Trägerlösung

- Szenario 4: Internationalisierungsprogramm

\section{Executive Summary}

The two "Offices of Science and Technology Austria" (OSTA) in Washington D.C. and Beijing aim to bring Austria closer to both leading and important nations in the field of Research, Technology and Innovation (RTI) as well as to support knowledge- and technology transfer beyond Europe. Both OSTA are located in the Austrian embassies, are led by a person from the diplomatic service and steered by four Federal Ministries according to a joint interministerial framework agreement (Federal Ministry for Europe, Integration and Foreign Affairs, Federal Ministry of Education, Science and Research, the Federal Ministry for Digitisation and Business Location and the Federal Ministry of Transport, Innovation and Technology). 


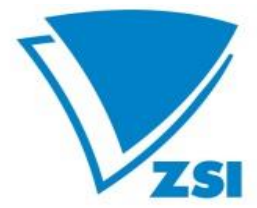

ZENTRUM FÜR SOZIALE INNOVATION

CENTRE FOR SOCIAL INNOVATION

The evaluation scrutinises the activities and their impact (including a comparison with two other European countries, Sweden and Switzerland), reviews the effectiveness and efficiency of the reporting and monitoring system, evaluates benefits and importance, proposes recommendations and designs four future scenarios.

The evaluation employed a multi-method approach, which included document analysis, face-to-face and telephone interviews, a survey, and focus groups. After all, a visit to each site was carried out to evaluate the activities and structures of the offices more precisely.

Both offices are actively engaged in their tasks, and the work programmes are in large part implemented. They are well interconnected with other (Austrian) organisations on site, the employees act service-oriented, competent and qualified. The majority of the persons and organisations that use OSTA services are also satisfied. Weaknesses and problems are apparent in the following respects:

- Interministerial governance is proving to be inflexible, complicated and time-consuming. The Ministries often miss to appropriate the specific profit for their own house.

- At OSTA Beijing, a great deal of time and energy is concentrated in the organization and support of delegation trips, and there are also overlaps with the activities of the AußenwirtschaftsCenters (foreign trade centres).

- OSTA Washington focuses on providing support and advice to researchers and to organise the annual ARIT. In particular, BMVIT does not see its interests as being represented in the light of this orientation.

- Local governance may cause conflicts of competence between the embassies, the OSTA management and the Ministries in charge.

- Due to their diplomatic status, the OSTA heads are only loosely associated with the Austrian RTI scene.

According to these findings, it is recommended to reduce the effort and make the current construction more flexible. The evaluation formulates the following recommendations:

- Clear positioning, definition of objectives, agenda and strategic orientation

- Improve the capability to connect with relevant actors and stakeholders in Austria 


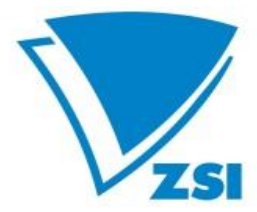

ZENTRUM FÜR SOZIALE INNOVATION

CENTRE FOR SOCIAL INNOVATION

- Define cooperation and demarcation with the AußenwirtschaftsCenters (foreign trade centres).

- Optimize reporting and introduce multi-year planning horizons

- Reduce transaction effort on all sides

Each future scenario should be designed in a way that the benefits for customers as well as motivation and commitment in the teams remain constant at least, while the satisfaction of the Ministries increases. The following scenarios are proposed by the evaluation:

Scenario 1: Small OSTA with Variable Geometry

Scenario 2: Office Community of Attachés

Scenario 3: Agency Solution

Scenario 4: Internationalisation Programme

\section{Ziele, Design und Methodik im Überblick}

\subsection{Ausgangssituation}

In den letzten Jahrzehnten ist internationale Zusammenarbeit zu einem immer wichtigeren Element nationaler und internationaler Forschungs-, Technologie- und Innovationspolitik (FTI Politik) geworden. Innerhalb Europas haben FTI Kooperationen eine lange Tradition und werden auf europäischer Ebene durch die Europäischen Rahmenprogramme für FTI durch die Europäische Kommission stark befördert. Mit der Einführung spezifischer ERA-Nets und der stärkeren Einbeziehung außereuropäischer Länder in EUREKA gab es vermehrt Möglichkeiten der Kooperation über Europa hinaus. Viele europäische Länder formulierten spezifische Strategien und schufen spezifische Unterstützungsformate für außereuropäische FTI-Kooperationen. Auch Österreich baute seine Förderungen für außereuropäische Zusammenarbeit in den letzten Jahren aus ${ }^{1}$. Die Errichtung der beiden OSTA in Washington und Peking sind im Kontext dieser intensivierten Kooperationsbemühungen zu sehen. Das OSTA Washington wurde bereits im Jahr 2001 gegründet

\footnotetext{
1 Siehe beispielsweise die BMVIT/FFG Kooperationsprojekte mit der chinesischen Akademie der Wissenschaften (CAS), das Kooperationsabkommen mit der Provinz Guangdong, die WTZ-Abkommen des BMBWF oder das Programm „Beyond Europe“ des BMDW, das auf Basis der Empfehlungen „Beyond Europe - Die Internationalisierung Österreichs in Forschung, Technologie und Innovation über Europa hinaus" erarbeitet wurde https://era.gv.at/directory/160/attach/FTI_AG7a_Brosch_re_Ansicht.pdf.
} 


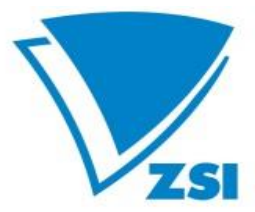

ZENTRUM FÜR SOZIALE INNOVATION

CENTRE FOR SOCIAL INNOVATION

und seit damals von der Motivation getragen, an die führende Forschungsnation USA anzudocken und die vielen österreichischen Forschenden vor Ort zu unterstützen. Die Einrichtung des OSTA Peking erfolgte sehr viel später, im Jahr 2012, als die Bedeutung der aufsteigenden BRICS-Staaten sich immer klarer herauskristallisierte. Die beiden Büros dienen der Stärkung der bilateralen Beziehungen mit Nordamerika und Mexiko sowie China und der Mongolei.

Die hier vorgelegte Evaluierung zeigt auf Basis einer Mischung aus qualitativen und quantitativen Methoden, wie die beiden OSTA dieser Aufgabe nachkommen, und formuliert Empfehlungen für die weitere Arbeit der Büros.

\subsection{Ziele und Methodik der Evaluierung}

Vor dem Hintergrund der in den Terms of Reference genannten Zielen der Evaluierung (Abschnitt III) zielt die vorliegende Evaluierung darauf ab,

- die Aktivitäten der beiden OSTA in Washington und Peking (Bereitstellung von Informationen, Vermittlung von Partnerschaften, Aufbau und Pflege von Netzwerken, Betreuung von Delegationsreisen, Organisation von ARIT, Abstimmung mit anderen Akteuren vor Ort und in Österreich, etc.) zu erfassen und zu bewerten,

- die Wirkung und die Nachhaltigkeit der Wirkung (auch im internationalen Vergleich mit anderen europäischen Ländern) zu erfassen und zu bewerten,

- das Reporting und Monitoring sowie die Governance in Österreich und den Sitzländern in Hinblick auf Effektivität und Effizienz zu überprüfen (Arbeitsprogramm, Selbstevaluation, Berichtswesen, Informationsaufbereitung, Büroorganisation, Lenkungsausschuss und verantwortliche Ministerien, Ressourceneinsatz und Gebarung),

- den Nutzen und die Bedeutung der Büros einzuschätzen

- und schließlich Empfehlungen für die weitere Arbeit zu formulieren.

Neben der Bewertung der operativen Tätigkeiten und der daraus resultierenden Wirkung stellt sich auch die Frage nach der strategischen Rolle der OSTA im Rahmen der nationalen Aktivitäten zur Internationalisierung des österreichischen FTI-Systems und deren Relevanz im Kontext anderer Aktivitäten und Maßnahmen (z.B. die Außenhandelsstrategie oder die Empfehlungen zur „Beyond Europe" Strategie). 


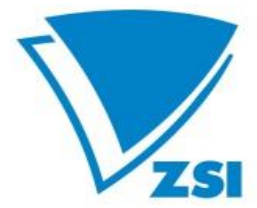

ZENTRUM FÜR SOZIALE INNOVATION

CENTRE FOR SOCIAL INNOVATION

Im Zuge der Evaluierung kamen die folgenden Methoden zum Einsatz:

- Ein Online-Survey adressierte Stakeholder, Organisationen und Personen, welche die Dienstleistungen der OSTA in Anspruch genommen haben (Forschende, Forschungseinrichtungen und Universitäten, Unternehmen, Teilnehmende ARIT) in Österreich und den beiden Sitzländern nach ihren Erfahrungen mit den Leistungen und Aktivitäten der OSTA sowie nach den Wirkungen und der Nachhaltigkeit der Wirkungen dieser Leistungen. Dieser Survey wurde auf Englisch und auf Deutsch erstellt.

- Interviews wurden vor allem mit den Bundesministerien sowie mit zentralen Stakeholdern und Akteuren in Österreich und den Sitzländern geführt, ebenso mit anderen europäischen Ländern für den internationalen Vergleich.

- Mittels Desk Research erfolgte eine Analyse der Materialien, der Berichte, der Arbeitsprogramme und der Gebarung. Auch bei den Recherchen zu den internationalen Beispielen (Schweden und Schweiz) wurden Großteils existierende Berichte und Materialien (inklusive Evaluierungsberichte) ausgewertet.

- Es wurden je ein Besuch vor Ort durchgeführt, um die Aktivitäten und Strukturen der Büros genauer bewerten zu können: Der Besuch in Washington fand vom 19. - 22. Mai 2019 statt, der Besuch in Peking vom 26. - 28. Mai 2019.

- Zwei Fokusgruppen mit Stakeholdern und institutionellen Akteuren in Österreich ergänzten und validierten die Ergebnisse der Einzelinterviews, der Besuche vor Ort, des Online-Surveys und der internationalen Beispiele. Die Fokusgruppe Washington wurde am 6. Juni 2019, die Fokusgruppe Peking am 14. Juni 2019 durchgeführt.

Die Teilnehmenden der Fokusgruppen sowie eine genaue Auflistung der interviewten Personen findet sich im Anhang A.

\section{Ergebnisse der Online Surveys}

Die Online-Surveys bildeten eine zentrale Grundlage für die Hypothesenentwicklung insbesondere für die Fokusgruppen sowie als Input für die Vor-Ort Besuche und Interviews an den jeweiligen Standorten. Es wurden zwei weitgehend identische Online-Surveys durchgeführt, die sich lediglich in der Frage nach spezifischen Leistungsbereichen des jeweiligen OSTA sowie in der geographischen 


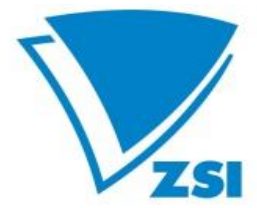

ZENTRUM FÜR SOZIALE INNOVATION

CENTRE FOR SOCIAL INNOVATION

Zuordnung unterschieden. Hervorzuheben ist, dass aus Datenschutzgründen Sampling und Versand der Online-Surveys durch die beiden OSTA selbst durchgeführt wurden, da entsprechende Kontaktdaten von Netzwerkmitgliedern und Stakeholder nicht direkt an das Evaluierungsteam weitergeben werden durften. Die Darstellung der entsprechenden Datengrundlagen, Rücklauf und Struktur werden im Folgenden für die beiden OSTA separat dargestellt. Insgesamt muss somit darauf hingewiesen werden, dass die jeweiligen Online-Surveys zwar für sich genommen wichtige Inputs und Erkenntnisse zu Bedeutung, Wirkungsweisen und Erwartungen an die jeweiligen OSTA lieferten, jedoch untereinander nicht direkt vergleichbar sind, da die entsprechenden Erhebungssamples unterschiedlich beschaffen waren. So waren im Fall des OSTA Washington Vertreterinnen und Vertreter von Ministerien sowie der nationalen Fördereinrichtungen FFG und FWF nicht im Sample enthalten.

Die Diskussion und Validierung der Survey-Resultate in den beiden Fokusgruppen zeigte jedoch klar, dass die entsprechenden Resultate insgesamt, aus Sicht der Teilnehmenden, ein plausibles Bild über KundInnen-Strukturen, Aktivitätsschwerpunkte und Außen-Wahrnehmungen der OSTA lieferten. Ein wichtiger Vorteil der Online-Surveys war auch Erreichung nicht-österreichischer OSTA Stakeholder in Nordamerika und China. Die Fragebögen wurden entsprechend jeweils auf Deutsch und Englisch angeboten.

Bei der Struktur der RespondentInnen wurden zwischen den Bereichen Unternehmensforschung, wissenschaftliche Forschung (Hochschulen), außeruniversitäre Forschung, Verwaltung wissenschaftlicher und außeruniversitärer Hochschulen- und Forschungseinrichtungen, Bundesministerien, Fördereinrichtungen sowie sonstige Akteure unterschieden.

\subsection{OSTA Washington}

Der Versand der Online-Survey zum OSTA Washington erfolgt zum einen über den Verteiler des RINANetzwerkes (zu dessen Zusammensetzung siehe Abschnitt 5.4). Zum anderen wurde die Einladung zur Survey auch über das ASciNA-Netzwerk gestreut, und zwar im Wege des Versands über das ASciNAPräsidium an die einzelnen Chapter-Heads. Wenig überraschend rekrutierte sich denn auch ein Großteil der Rückmeldungen aus dem Bereich der wissenschaftlichen Forschung. Personen aus den österreichischen Bundesministerien sowie Förderagenturen konnten auf diesem Wege leider nicht erreicht werden, da diese in keinem der beiden Verteiler enthalten waren. Jedoch konnten amerikanische Stakeholder aus Förderung und Politik erreicht werden. 


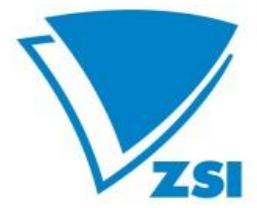

ZENTRUM FÜR SOZIALE INNOVATION

CENTRE FOR SOCIAL ININOVATION

Insgesamt wurden 73 vollständig ausgefüllte Fragebögen gewertet, 17 davon mit der Angabe niemals direkt in Kontakt mit OSTA gewesen zu sein beendet.

Etwa 75\% der RespondentInnen entstammten dem Bereich der wissenschaftlichen Forschung (sowohl Hochschulen als auch außeruniversitäre Forschung), 3\% entfielen auf den Verwaltungsbereich wissenschaftlicher und außeruniversitärer Forschungsinstitutionen (Abbildung 1). Der Rest entstammte zu gleichen Teilen Unternehmen sowie sonstigen Akteuren, wobei zu letzteren Rückmeldungen des National Institute of Health (NIH), der US Regierung sowie der Vereinten Nationen zählen.

Häufigste Aktivitätsbereiche umfassten im wissenschaftlichen Bereich, eingedenk der Struktur der RespondentInnen, mit Abstand Forschungsaufenthalte in den USA. Weniger als die Hälfte war im Vergleich dazu in der Anbahnung bzw. Durchführung kooperativer Forschungs- und Innovationsprojekte aktiv. Häufigste Aktivitätsbereiche der Unternehmen umfassten hingegen die Anbahnung von Forschungskooperationen sowie die Anbahnung wirtschaftlicher Kooperationen (siehe 10.4).

Abbildung 1: Verteilung RespondentInnen: OSTA Washington; N=73

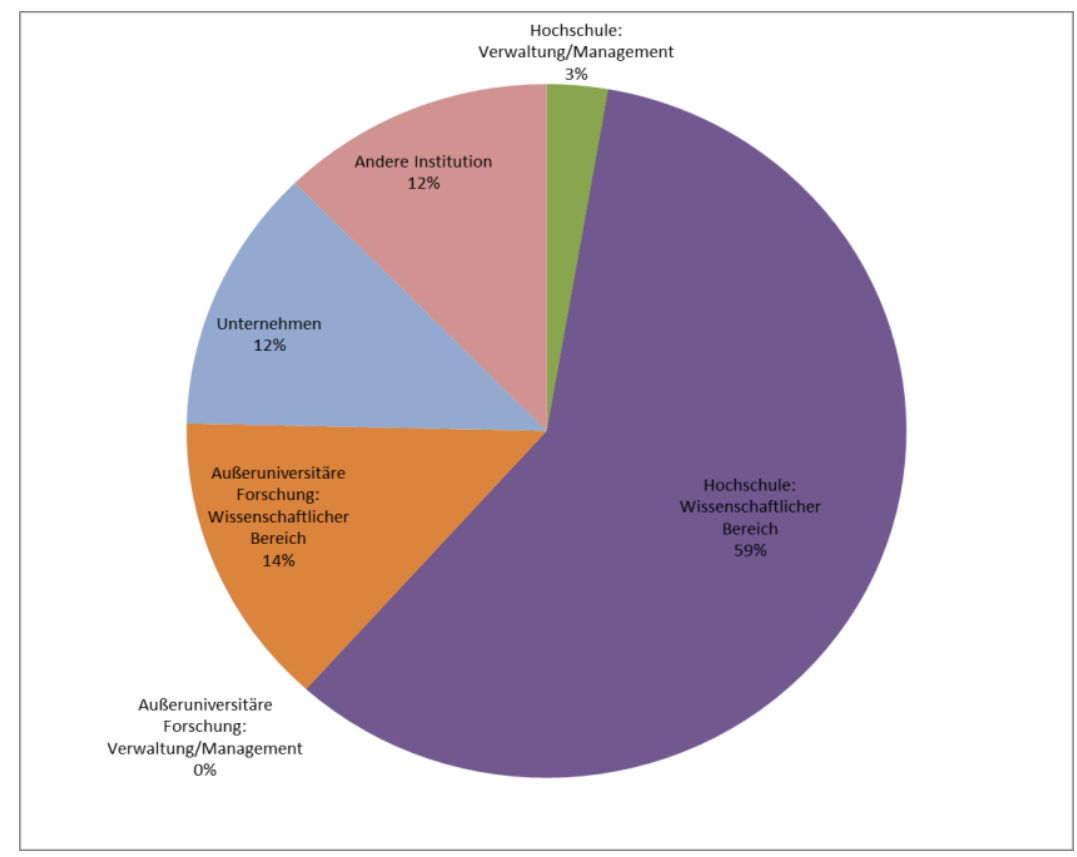

Quelle: JOANNEUM RESEARCH 


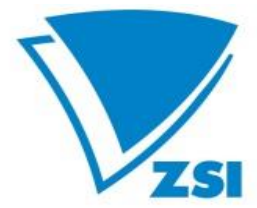

ZENTRUM FÜR SOZIALE INNOVATION

CENTRE FOR SOCIAL INNOVATION

\subsection{OSTA Peking}

Grundlage für das Sampling und den Versand des Online-Survey zu OSTA Peking waren der Newsletter-Verteiler des OSTA Peking sowie die Alumni-Datenbank. Ersterer beinhaltete interessierte Forschende, Unternehmen, Stakeholder und Intermediäre sowie Vertretungen der zuständigen Bundesministerien. In der Alumni-Datenbank waren zudem Kontakte zu chinesischen Studierenden und Forschenden mit Aufenthalt in Österreich enthalten. Eine Herausforderung stellte die Erfassung von Teilnehmenden aus Forschung und Wirtschaft an Delegationsreisen nach China dar. Diese werden über den Newsletter-Verteiler des OSTA Peking nur dann erfasst, wenn sie sich aktiv registrieren. Deren Anteil lässt sich aber aus den Daten nicht herauslesen. Um die Teilnehmenden an Delegationsreisen bestmöglich zu adressieren wurde parallel zum Versand über die OSTA-eigenen Verteiler, eine Einladung zur Teilnahme an der Erhebung durch die FFG versandt, welche über die Kontaktdaten aller Teilnehmenden an Delegationsreisenden verfügt. Nach aktiver Zustimmung der Personen wurde der Fragebogen durch JOANNEUM RESEARCH versandt. Auf diesem Wege konnten zusätzlich fünf Personen erreicht werden.

Insgesamt wurden 35 vollständig ausgefüllte Fragebögen gewertet, 9 davon mit der Angabe niemals direkt in Kontakt mit OSTA gewesen zu sein.

Abbildung 2 stellt die Verteilung der RespondentInnen der Erhebung zur OSTA Peking dar. Etwa ein Drittel entstammen dabei der wissenschaftlichen Forschung (sowohl Hochschulen als auch außeruniversitäre Forschung), 20 \% Unternehmen, 11 \% Verwaltungsbereich von Universitäten sowie außeruniversitären Forschungseinrichtungen. Einzeln geantwortet haben Ministerien, die WKO sowie FFG und FWF.

Befragt nach den häufigsten Aktivitätsbereichen gaben Akteure der wissenschaftlichen und außeruniversitären Forschung den Aufbau kollaborativer F\&I-Infrastrukturen und F\&EProjektanbahnungen mit chinesischen Akteuren sowie den Forschenden Austausch als wichtigste Aktivitätsbereiche in China an. Der häufigste Aktivitätsbereich von Unternehmen war mit Abstand die Anbahnung wirtschaftlicher Kooperation in China (siehe Annex 10.4). 

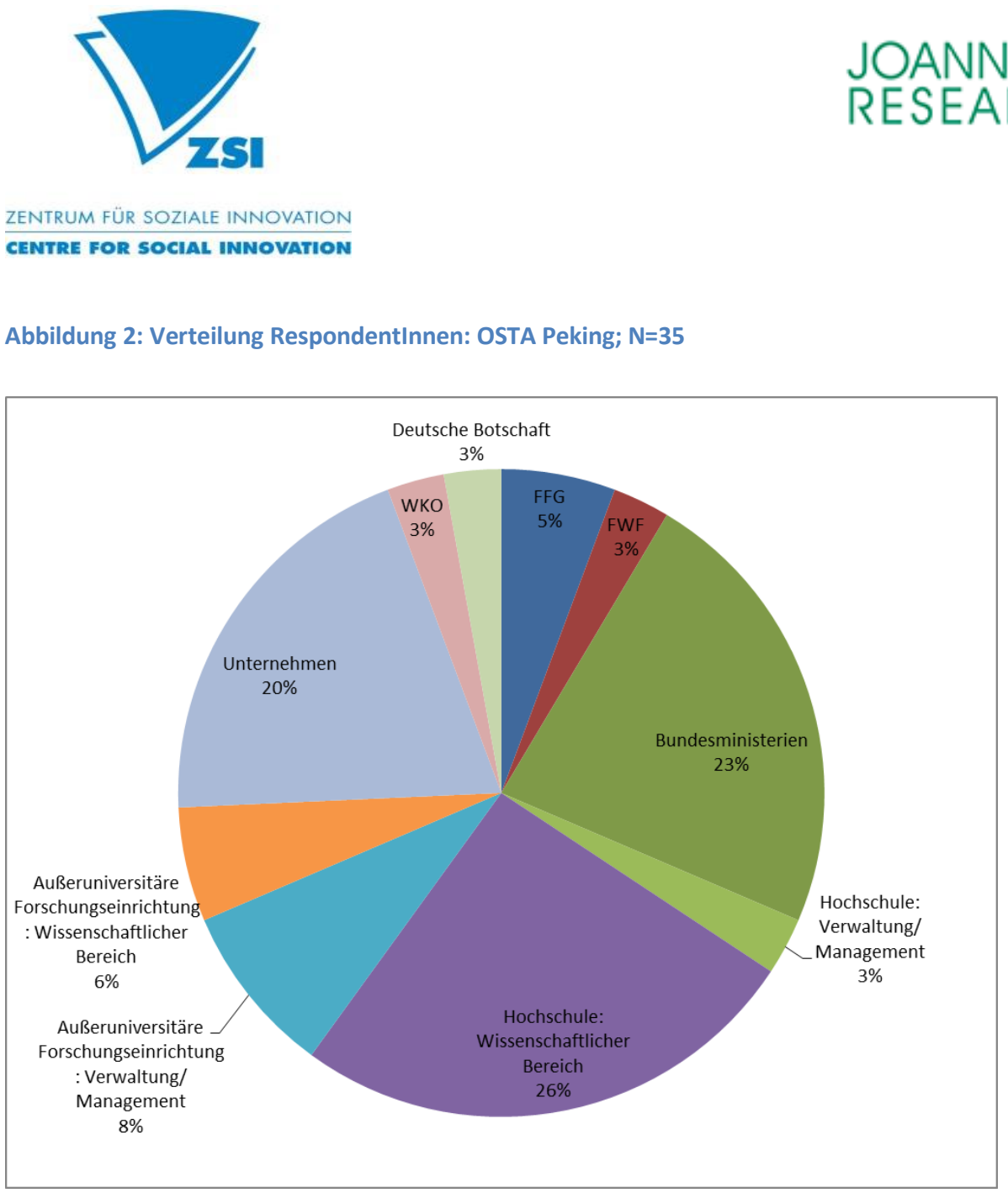

Quelle: JOANNEUM RESEARCH

Im Folgenden werden zentrale Ergebnisse der beiden Online-Surveys zusammenfassend dargestellt. Die entsprechenden Darstellungen finden sich im Annex. Dies erfolgt zum Zweck der Illustration und transparenten Darstellung der Rohergebnisse. Die Einbindung der Ergebnisse, Gewichtung der Aussagen und Interpretation erfolgt in weiterer Folge entlang der jeweiligen Evaluierungsschwerpunkte und Fragestellungen.

\subsection{OSTA Washington - zentrale Ergebnisse}

Die Bekanntheit des OSTA rührt in erster Linie (mit Abstand) von der Vermittlung durch Veranstaltungen und Netzwerkevents sowie über ASciNA her.

In Bezug auf Frage nach der Relevanz der Leistungsbereiche des OSTA wurden, wenig überraschend eigendenk der RespondentInnen-Population, die Beratung und Betreuung österreichischer Forschenden vor Ort sowie die Unterstützung der internationalen Mobilität von Forschenden, als mit Abstand wichtigste Leistungsbereiche identifiziert. Dahinter folgten etwa gleich auf die Bereiche „Herstellung von Kontakten", „Unterstützung bi- und multilateraler Kooperationen" sowie "Datenund Informationsbereitstellung zu den USA“. Aktivitäten im Bereich der Unternehmensforschung und 


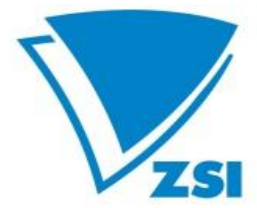

ZENTRUM FÜR SOZIALE INNOVATION

CENTRE FOR SOCIAL INNOVATION

Technologvermarktung lagen leicht dahinter, allerdings mit wesentlich höheren Enthaltungszahlen bei der Beurteilung der Relevanz. In-Flow-Aktivitäten amerikanischer Akteure nach Österreich wurden weniger häufig als relevant beurteilt.

Dort wo entsprechende Leistungen in Anspruch genommen wurden, waren die RespondentInnen mehrheitlich „Zufriedener" bis „Sehr zufrieden".

Bei der Beurteilung der RINA-Leistungen fällt auf, dass die Bekanntheit des RINA-Netzwerkes unter den Befragten hinter jener von ASciNA zurückliegt (37 Personen zu 46). Die Fallzahlen zu den weiterführenden Fragen zu RINA waren dann nochmals um einiges geringer, weswegen auf die Auswertung der Zufriedenheit verzichtet wurde. Unter jenen die sich zu RINA geäußert haben, sind das Network Event ARIT sowie die Webauftritte des Netzwerkes die bekanntesten Leistungsbereiche. Die administrativen Beratungsleistungen (Double Taxation, Dual Citizenship) erscheinen dagegen eher unbekannt.

43 Personen verneinten die Frage, ob OSTA die wichtigste Informations-/Unterstützungsquelle für ihren USA Aufenthalt gewesen sei $(\mathrm{N}=54) .11$ beantworteten dies mit „Ja“. In Bezug auf anderweitig in Anspruch genommene Finanzierungs- und Beratungsleistungen sticht der FWF mit Abstand hervor, gefolgt von Fulbright-Stipendien und ERASMUS.

Eingedenk der bereits beschriebenen Bedeutung, die der Betreuung österreichischer Forschender in den USA beigemessen wird, ist wenig überraschend, dass dieser Bereich auch als in der Zukunft wichtigste angesehen wird (40 Zustimmungen, $\mathrm{N}=55$ ). Dahinter folgen mit gewissem Abstand die Vermittlung von Jobangeboten sowie die Unterstützung bi- und multilateraler Kooperationen.

Die Auswertung der offenen Fragen zu Funktion und Zukunft des OSTA zeigen ein, eingedenk der Struktur der RespondentInnen, mehrheitlich positives Bild zur Bedeutung des OSTA für die österreichische Forschungsgemeinschaft wobei mehrheitlich für dessen Fortbestand plädiert wird.

An zwei Stellen wurde jedoch auf eine notwendige Verbesserung in Bezug auf Anbindung an das „offizielle Österreich“ sowie eine aktuell zu starke Fokussierung des OSTA auf Naturwissenschaften im Vergleich zu Kultur und Geisteswissenschaften verwiesen.

- „Fuer die Aussenwirkung und Verbindung zum "offiziellen" Oesterreich wichtig. Fuer Wissenschaftlerlnnen in den USA ist ASciNA der bessere Ansprechpartner da von Wissenschaftern fuer Wissenschafter." (Unternehmensrückmeldung) 


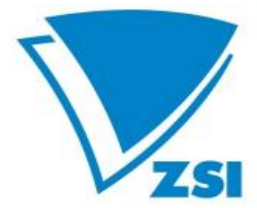

ZENTRUM FÜR SOZIALE INNOVATION

CENTRE FOR SOCIAL INNOVATION

Interessant war die Beurteilung zweier amerikanischer RespondentInnen (NIH und Government) wonach es OSTA aus Sicht der USA an einer klaren Agenda und einem strukturierten Außenauftritt in Bezug auf die Verfügbarkeit als Ansprechpartner und die Rolle als Knotenpunkt zur nationalen FTIPolitik mangle.

- "The lack of support from the Austrian government for the OSTA office in DC makes this seemingly hard sometimes" und in Bezug auf die Vermarktung österreichischer Technologien und Anbahnung von Kooperationen zwischen Forschungseinrichtungen und Unternehmen "I have come across a lot of opportunities for German researchers but never see anything for Austrian researchers."

Das erweckt etwas den Eindruck, dass die Anbindung des OSTA an amerikanische Akteure möglicherweise kritisch zu sehen ist, die vor Ort interviewten Organisationen bestätigten dies allerdings nicht.

\subsection{OSTA Peking - zentrale Ergebnisse}

Wie bei OSTA Washington rührt die Bekanntheit des OSTA Peking in erster Linie (mit Abstand) von der Vermittlung durch Veranstaltungen und Netzwerkevents her.

Die Beantwortung der Frage nach der Bedeutung der Leistungsbereiche des OSTA Peking verdeutlicht dessen unterschiedliche Struktur und Stoßrichtung im Vergleich zum OSTA Washington. So Sind hier Delegationsreisen, das Herstellen von Kontakten, die Unterstützung bilateraler FTI-politischer Kooperationen sowie die Informationsbereitstellung von Daten und Fakten zu China im Verhältnis zu einander deutlich prioritärer gereiht als für das OSTA Washington. Gleichwohl wird im Vergleich dazu auch der Mobilitätsunterstützung von Forschenden eine hohe Priorität eingeräumt.

Interessant ist, dass auch in der gesonderten Auswertung für Hochschulen und Forschungseinrichtungen die Leistungsbereiche der Delegationsreisen aber auch das Herstellen von Unternehmenskontakten, in Bezug auf ihre Relevanz vergleichsweise hoch gereiht wurden. Interessant ist auch, dass, anders als für das OSTA Washington, auch Incoming-Aktivitäten chinesischer Forschender und Unternehmen durchaus eine gleichwertige Rolle im Leistungsspektrum zu spielen scheinen (soweit man das auf Basis der geringen Fallzahlen sagen kann).

Insgesamt scheint, soweit beantwortet, auch die Zufriedenheit mit den Leistungen durchwegs gegeben zu sein, wenngleich sich hier auch eine gewisse Diskrepanz im Verständnis um die Ausrichtung des OSTA zeigt: So wurde einerseits angemerkt, dass sich das OSTA verstärkt auf die 


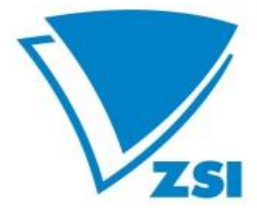

ZENTRUM FÜR SOZIALE INNOVATION

CENTRE FOR SOCIAL INNOVATION

Vermarktung österreichischer Technologien in China konzentrieren solle. Andererseits wurden Verbesserungen in Unterstützung von Forschenden und der Alumni-Betreuung gefordert.

- Unternehmen: „Reduktion des Aufgaben- bzw. Zuständigkeitsbereiches, Fokus auf Unterstützung österr. Technologiefirmen in China"

- Chinesische Hochschule: "Promote the bilateral cooperation, or facilitate bilateral joint research projects at academic/scientific area"

In Bezug auf die Inanspruchnahme sonstiger Unterstützungsleistungen stechen insbesondere Leistungen des OeAD, der WKO sowie des BMBWF hervor. Interessant ist, dass die FFG-Programme vergleichsweise wenig von den RespondentInnen herangezogen wurden, wobei dies auch dem Sampling der Erhebung geschuldet sein kann (es kann nicht abgeschätzt werden, inwieweit Teilnehmende an Tecxport wirklich erreicht wurden).

In Bezug auf künftig zu vertiefende Leistungsbereiche wurden die Unterstützung des Ausbaus bilateraler Kooperationen auf (FTI-)politischer Ebene, die Unterstützung bi- und multilateraler Kooperationen (z.B. in EU-Programmen), die Unterstützung internationaler Mobilität (Austausch) von Forschenden, Anbahnung bilateraler Kooperation zwischen Forschungseinrichtungen und Unternehmen, Identifikation von potenziellen Partnern und Herstellen von Kontakten sowie Informationsbereitstellung, Analysen und Daten zu China genannt.

Interessant ist auch hier, dass im Bereich Hochschulen und Forschungseinrichtungen Informationsbereitstellung, Analysen und Daten zu China sowie die Anbahnung bilateraler Kooperation zwischen Forschungseinrichtungen und Unternehmen ganz vorne gereiht sind.

Hohe Bedeutung wird dem OSTA Peking insbesondere aus Sicht wirtschaftlicher Akteure und Stakeholder beigemessen:

- „In dem schmalen Segment "Unterstützung ö. Technologiefirmen" äußerst hilfreich, das restliche Spektrum für uns nicht relevant"

- „Weil das OSTA in diesem Bereich die AWO/WKO punktuell gut unterstützen und damit einen Mehrwert erbringen kann"

Auch wird dessen diplomatische Brückenfunktion in der FTI-Politik zwischen Österreich und China, sowie die entsprechende Unterstützung beiderseitiger Akteure hervorgehoben: 


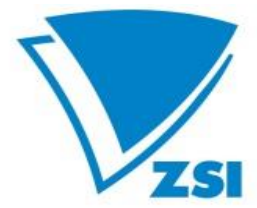

ZENTRUM FÜR SOZIALE INNOVATION

CENTRE FOR SOCIAL INNOVATION

- Chinesische Hochschule: „OSTA is a window to know more about alumni, and multinationals of Austria"

- "Hohe Bedeutung, da es wichtig ist, eine Anlaufstelle in China zu haben, mit Mitarbeiterinnen, die mindestens zweisprachig sind. Direkte Kontakte zu MinisterienvertreterInnen sind damit viel einfacher. Zudem erhält man Informationen, die man, aufgrund der sprachlichen Barrieren, nicht bekommen würde."

- [Relevanz] „sehr hoch im Aufbau der Kooperation mit CAS und Uni Shanghai“

- Außeruniversitäre Forschungseinrichtung Verwaltung: „Hohe Relevanz ist gegeben. Kontakt vor Ort in China enorm hilfreich. Unterstützungsleistung bei organisatorischen Fragestellungen sehr wichtig."

Kritische Stimmen zum OSTA Peking betrafen in erster Linie die als unzureichend wahrgenommene Rolle des OSTA als proaktiver Informationsanbieter zu Entwicklungen in China.

- „Aufbereitung der Informationsmaterialien - wünschenswert wäre, ohne Aufforderung Berichte über aktuelle Entwicklungen in China zu erhalten (politische Entwicklungen, etc.)"

- „Screening der FTI politischen Entwicklungen und konkrete Aufbereitung für Österreich inkl. Handlungsempfehlungen für Stakeholder in Österreich"

- „ „[...] Bedarf an strategischen FTI Einschätzungen wird aber nicht gedeckt"

\section{Internationale Beispiele}

\subsection{Schweden}

Für Schweden sind vier verschiedene Organisationen international aktiv, die teilweise sehr eng zusammenarbeiten:

- Offices of Science and Innovation

- Vinnova

- Team Sweden

- The Swedish Institute

Die „Offices of Science and Innovation“ sind ähnlich den österreichischen OSTA an ausgewählten Botschaften angesiedelt. Die aktuellen Standorte befinden sich in Tokyo, Seoul, Brasilia, Washington, New Delhi und Beijing. Ebenfalls ähnlich der OSTA werden sie von mehreren Ministerien gemeinsam 


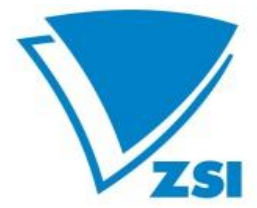

ZENTRUM FÜR SOZIALE INNOVATION

CENTRE FOR SOCIAL INNOVATION

finanziert und inhaltlich verantwortet: Dem Ministry of Enterprise and Innovation, dem Ministry of Infrastructure, dem Ministry of Education and Research und dem Ministry of the Environment, in enger Zusammenarbeit mit dem Ministry for Foreign Affairs.

Gemäß den von der Regierung definierten "Innovation Partnership Programmes" sind die Offices in fünf Schwerpunktbereichen tätig: "Next Generation Travel and Transport, Smart Cities, Circular and Bio-based Economy, Life Science and Connected Industry, New Materials". Es bestehen explizite Verbindungen zur "2030 Agenda for Sustainable Development" und zu den globalen Zielen der UN.

Die Standortteams bestehen jeweils aus einem "Officer" und drei bis vier Mitarbeitenden. Sie tauschen sich regelmäßig untereinander aus und betreiben einen gemeinsamen Blog: https://sweden-science-innovation.blog/

Vinnova, eine mit der FFG vergleichbare Agentur für angewandte Forschung und Innovation, betreibt Filialen in Palo Alto und seit neustem auch in Tel Aviv. In Palo Alto arbeitet Vinnova unter dem Dach des Nordic Innovation House (gemeinsam mit Norwegen, Finnland, Dänemark, Island) mit Schwerpunkt auf Start-ups:

- Incubator Programme (TINC),

- Accelerator Programme (REACH) sowie

- Internship-Programme (Praktikumsplätze),

Vinnova Tel Aviv wurde erst kürzlich gegründet (operativer Start 2019) und konzentriert sich ähnlich wie in Palo Alto auf Start-ups.

Team Sweden wiederum ist ein Netzwerk aus Regierungsbehörden und Agenturen (19 Mitglieder), die Forschung und Innovation, Wirtschaft und Export wie auch allgemein Internationalisierung fördern. Die Teams sind in Brasilien, China, und Indien tätig und auf die Themen Cleantech, Food, Energy, Care \& Health, Cultural Creative Businesses fokussiert, unterstützen aber auch Meetings \& Events sowie Investitionen aller Branchen und Technologiebereiche.

Bei The Swedish Institute schließlich handelt es sich um eine öffentliche Agentur, die schwedische Interessen in Kultur, Bildung, Wissenschaft, Wirtschaft und Entwicklungshilfe global repräsentiert. 


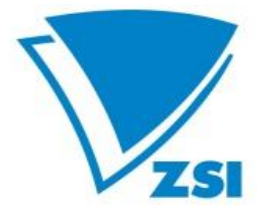

ZENTRUM FÜR SOZIALE INNOVATION

CENTRE FOR SOCIAL INNOVATION

Eine im Jahr 2018 von der schwedischen Regierung beauftragte Studie „Ökad attraktionskraft för kunskapsnationen Sverige (2018)" empfiehlt eine deutliche Ausweitung und Intensivierung der Internationalisierung von Forschenden, Hochschulen und Wissenschaft.

- Stärkung und Ausbau der Offices of Science and Innovation

- Pilotprojekt mit zwei neuen Offices im Schwerpunkt "Higher Education"

- Ausbau des Swedish Institute ("Sweden as a knowledge nation")

- Initiierung eines Programms "Swedish research and higher education ambassadors" (schwedische Forschende im Ausland können sich als "Ambassadors" bewerben)

- Stärkere Berücksichtigung von Universitäten und Forschungsförderungsorganisationen innerhalb der Team Sweden Gruppen

\subsection{Schweiz}

Die „Internationale Strategie der Schweiz im Bereich Bildung, Forschung und Innovation" aus dem Jahr 2018 fasst Visionen, Zielsetzungen und Umsetzungsschritte in der Schweizer FTI-Politik zusammen, welche in Bezug auf Maßnahmen, Programmen und Tätigkeitsgebiete in den Geltungsbereich des Staatsekretariats für Bildung, Forschung und Innovation fallen (SBFI).

Schwerpunkte der Internationalen Strategie ${ }^{3}$ der Schweiz im Bereich Bildung, Forschung und Innovation (ERI) 2018 des SBFI sind dabei die Förderung der:

- Grenzüberschreitenden Mobilität

- Internationalen Bildungs- und Berufsbildungszusammenarbeit

- Internationalen Forschungs- und Innovationszusammenarbeit

- Vernetzung BFI-Akteure

- Forschung im Bereich Raumfahrt

\footnotetext{
${ }^{2}$ Für eine englische Zusammenfassung siehe https://www.regeringen.se/4ab6ff/contentassets/2cf334f2792c42d4b8c414076eb59204/summary-in-english-internationalisation-ofswedish-higher-education-institutions

${ }^{3}$ Schweizer Eidgenossenschaft (2018): Internationale Strategie der Schweiz im Bereich Bildung, Forschung und Innovation; Strategie des Bundesrats, Juli 2018; Bern.
} 


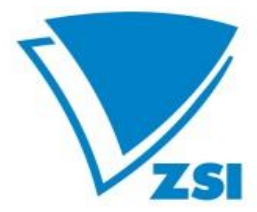

ZENTRUM FÜR SOZIALE INNOVATION

CENTRE FOR SOCIAL INNOVATION

- Beteiligung an internationalen Organisationen

Die Schweiz hat in der internationalen Zusammenarbeit eine lange Tradition bi- und multilateraler Kooperationen Schweizer FTI-Intermediäre und Akteure. Beispiele hierfür sind:

- SNF: Bilateral Programms, Lead Agency Verfahren, research for development (r4d) programme, Individuelle Mobilitätsunterstützungsleistungen

- Innosuisse: Internationalisation Camps (in Koop. mit swissnex), International Research Networks, EEN, EUREKA

- Partizipation an EU-Forschungsrahmenprogramm und EU-Partnerschaften als assoziierter Staat

Besonders hervorgehoben werden im Rahmen der Strategie, neben den bi- und multilateralen Kooperationen der Schweiz auf Programm-Ebene, auch eine Reihe institutionalisierter Maßnahmen und Initiativen. Zu diesen institutionalisierten transnationalen FTI-Infrastrukturen und Akteuren zählen insbesondere:

- swissuniversities: Unterstützung des Studierenden- und Forschenden-Austausch

- swissnex: Internationalisierung und Promotion der Schweizer FTI

- Swisscore: Verbindungsbüro in Brüssel, getragen von SBFI, SNF und Innosuisse

\subsubsection{Das swissnex-Modell}

swissenex ist ein zentraler Bestandteil des Schweizer Außennetzes für Bildung, Forschung und Innovation (BFI-Außennetz) mit folgenden zentralen Zielsetzungen:

- Internationale Vernetzung der Schweizer BFI-Akteure fördern

- Visibilität des Schweizer BFI-Standortes erhöhen

- Information, Beratung und Expertise zum Sitzland bieten

- Förderung von Wissensaustausch \& Inspiration (Delegationsreisen, Study Tours etc.)

Die Organisationsform der swissnex geht auf das 2000 gegründete Swiss House for Advanced Research and Education (SHARE) in Boston zurück, bei dem erstmals der neuartige Public-PrivatePartnership-Ansatz in der Gestaltung eines Schweizer "Wissenschaftskonsulats“ evident wurde. SHARE wurde als Plattform in enger Partnerschaft mit zentralen Schweizer BFI-Akteuren aus dem Hochschul- und Forschungssektor und innovativen Privatunternehmen errichtet. Dieser Ansatz 


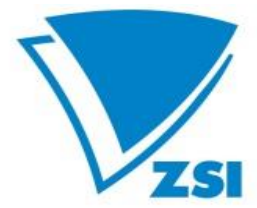

ZENTRUM FÜR SOZIALE INNOVATION

CENTRE FOR SOCIAL INNOVATION

spiegelt sich auch in der Organisationsform der swissnex-Initiativen wieder. Dabei sind grundsätzlich zwei Strukturen zu unterscheiden:

- swissnex Standorte (inkl. Satelliten): diese sind in regional und global führenden Innovationsmetropolen, aufstrebenden Märkten mit wirtschaftlichem und wissenschaftlichem Potenzial angesiedelt. Im Vordergrund der Auswahl steht dabei die Bedeutung des Standortes für die jeweiligen Partner; das Hauptstadtprinzip ist nicht maßgeblich.

WissenschaftsrätInnen: Diese sind als Fachspezialistlnnen für BFI-Themen in den Botschaften wichtiger BFI-Partnerländer angesiedelt. Diese werden je nach Standort vom SBFI bzw. vom Eidgenössischen Department für auswertige Angelegenheiten finanziert.

Aktuell existieren fünf swissnex Standorte in Boston, San Francisco, Shanghai, Bangalore, Rio de Janeiro, mit drei, den jeweiligen Hauptstandorten zugeordneten Außenstellen in New York, Sao Paolo und Guangzhou. An 22 Botschaften weltweit sind zudem WissenschaftsrätInnen aktiv.

\section{Abbildung 3: swissnex-Netzwerk}

\section{-Standorte/Satelliten -WissenschaftsrätInnen -Headquarter Bern}

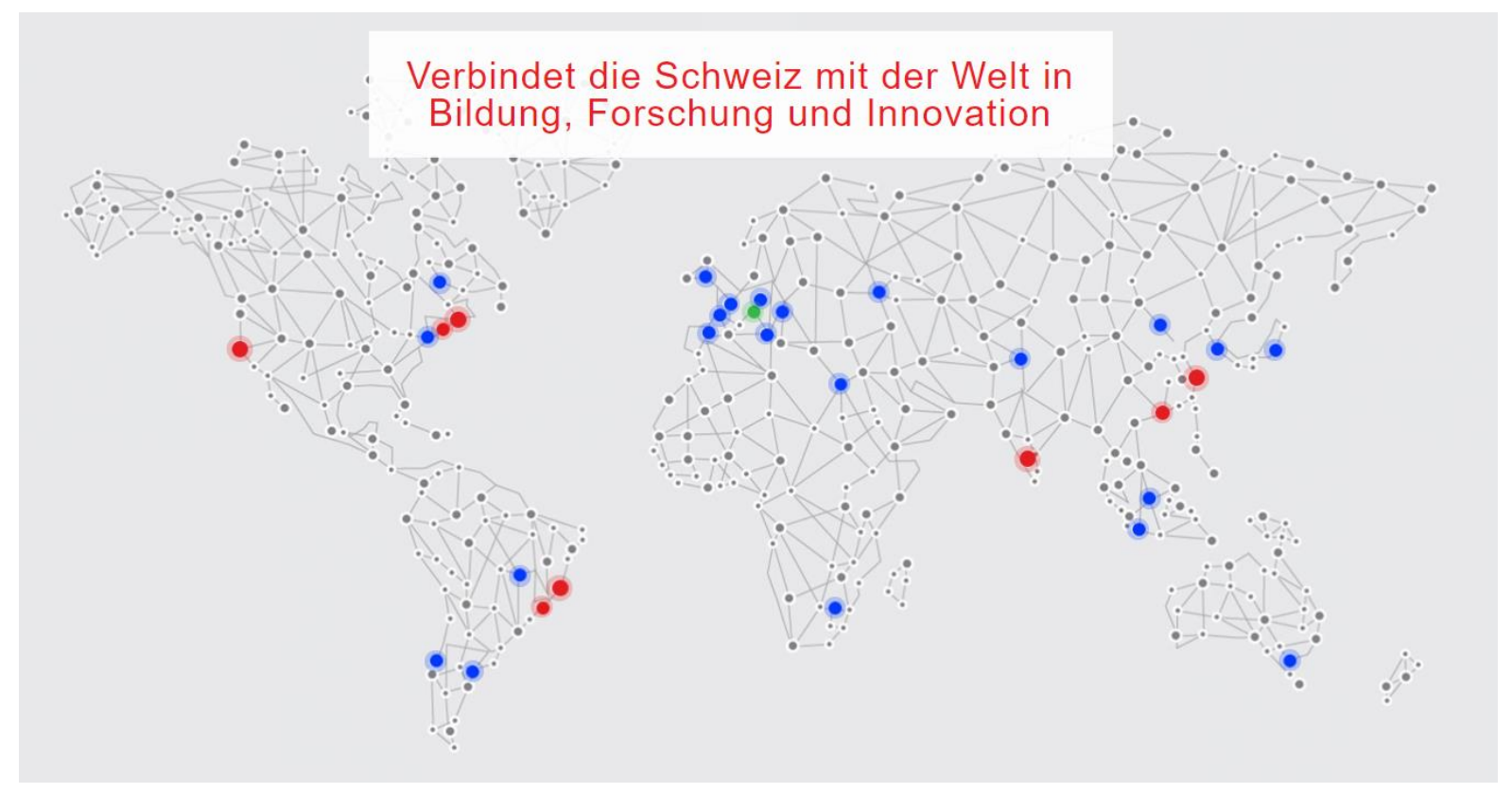

Quelle: swissnex 2019. 


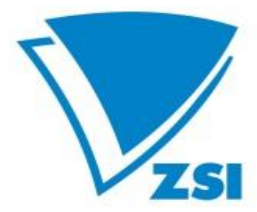

ZENTRUM FÜR SOZIALE INNOVATION

CENTRE FOR SOCIAL INNOVATION

\subsection{2. swissnex-Standorte - Organisationsform und Finanzierung}

swissnex agiert unter fachlicher und operativer Führung des SBFI in enger Kooperation mit dem Department für auswärtige Angelegenheit (EDA). Inhaltlich koordiniert und geführt werden die swissnex durch die Abteilung Internationale Beziehungen des SBFI. Administrativ ist swissnex an das Schweizer Außennetz des EDA angegliedert (Botschaften, Konsulate) und haben damit den Status offizieller Vertretungen.

Die swissnex-Standorte basieren auf folgenden zentralen Organisationsprinzipien: ${ }^{4}$

Strategische Standortwahl: Die swissnex Standorte situieren sich in regional und global führenden Innovationsmetropolen wie auch in aufstrebenden Märkten mit bedeutendem wirtschaftlichem und wissenschaftlichem Potenzial. Die Wissenschaftsrätinnen und -räte befinden sich dementgegen in den politischen Kapitalen für die bilaterale Kooperation wichtiger BFI-Partnerländer.

Partnerschaftliches Finanzierungsmodell: Partner der swissnex Standorte sind öffentliche wie private Akteure der schweizerischen und lokalen Bildungs-, Forschungs- und Innovationslandschaft. Im Rahmen des PPP-Finanzierungsmodells sind mindestens zwei Drittel der Ressourcen für Aktivitäten und Projekte der swissnex von wirtschaftlichen und wissenschaftlichen Partnerorganisationen in der Schweiz bzw. vor Ort aufzubringen. Dies kann sowohl über monetäre als auch in-kind-Leistungen erfolgen. Die Akquisition von Partnern und die Drittmittelakquise liegen in der Eigenverantwortung der jeweiligen Standorte.

Autonomie und dezentralisierte Governance: Das swissnex-Netzwerk baut auf schlanken Führungsstrukturen und eine dezentralisierte Governance auf. Das SBFI verantwortet u.a. die Gesamtstrategie, die Zielvorgaben, das Controlling und die Führung der swissnex Leiterinnen und leiter. Die Leistungssteuerung erfolgt im Wege vierjähriger Leistungsvereinbarungen (Service Level Agreements) mit SBFI und jährlichen Prioritätensetzungen (Lettre de Mission) als Grundlage für die Gewährung der Basisfinanzierung durch das SBFI. Die eigenständige Mittelverwaltung und Akquise wird mittels externer Audits evaluiert.

Unternehmerische Organisationskultur: Durch weitestgehend autonome Entscheidungsstrukturen und Prioritätensetzungen innerhalb der swissnex-Standorte, steht die Eigenverantwortung der

${ }^{4}$ SBFI (2015): Eine Roadmap für die Weiterentwicklung des swissnex Netzwerkes; Bern 2015. 


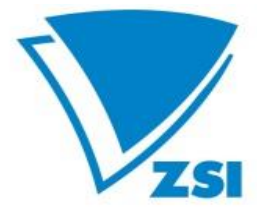

ZENTRUM FÜR SOZIALE INNOVATION

CENTRE FOR SOCIAL INNOVATION

MitarbeiterInnen, inbs. der LeiterInnen, im Vordergrund. Lean Management, d.h. die Serviceorientierte, eigenverantwortliche Optimierung von Tätigkeiten und Prozessen, wird dabei als Organisationsprinzip angeführt. Dies umfasst auch die eigenständige Netzwerkentwicklung und Pflege sowie die Personalentwicklung.

Partnerschaften im Rahmen des swissnex-Modells können über die direkte Beteiligung im Rahmen der PPP hinaus unterschiedliche Formen annehmen. Dazu zählen bspw. philanthropische und Sponsoring-Aktivitäten. Auch die Beauftragung der swissnex zur Erbringung gewisser entgeltlicher Dienstleistungen für eine öffentliche oder private Institution im Rahmen eines Mandatsvertrages sind möglich. Darüber hinaus können sich flexible Konsortien zur Durchführung gemeinsamer Projekte mit swissnex bilden. Ein Beispiel ist die Nutzung des swissnex als Residency bzw. Co-Working Space für Partnerinstitutionen mit BFI-Aktivitäten zwischen der Schweiz und dem swissnex Standort. Basierend auf einem Mandatsvertrag können Arbeitsplätze im Co-Working Space des swissnex für VertreterInnen der BFI-Partnerinstitution für einen bestimmten Zeitraum eingerichtet werden.

Ein zentrales Element zur Verankerung von swissnex in der Schweizer BFI-Landschaft einerseits, sowie zur Weiterentwicklung des Schweizer Außennetzes andererseits, ist das swissnex Committee, ein aktuell 9-köpfiges Gremium, bestehend aus Mitgliedern von swissnex-Partnerorganisationen, BFIIntermediären, Politik und den zuständigen Departments. Das swissnex Committee fungiert als strategisches Beratungsgremium für den Staatssekretär im SBFI.

\subsubsection{Zentrale Take-Aways}

Die Evaluierung des swissnex Modell durch das SBFI im Jahr 2015 liefert zum einen wichtige Anhaltspunkte über die Funktionsweise und Effektivität der Initiative aus Schweizer Perspektive. Gleichzeitig lassen sich daraus jedoch auch Schlussfolgerungen für eine Weiterentwicklung des Modells der OSTA ableiten.

Hier ist zunächst das PPP-Finanzierungsmodell und die eigenverantwortliche Mittelverwaltung zu nennen, die sowohl zur Erhöhung der Effizienz und zum Commitment der Partner positiv beitragen. Auch die geographische Streuung der swissnex nach den Prinzipien der Relevanz für die jeweiligen Partner sowie die Erwartungen hinsichtlich der Entwicklung der Region in Bezug auf Innovationen und wirtschaftliche Potenziale, kann als Anhaltspunkt für die Entwicklung von Kriterien zur Standortentscheidung entsprechender Einrichtungen dienen. In diesem Zusammenhang ist auch die 


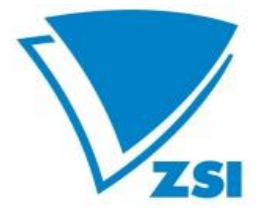

ZENTRUM FÜR SOZIALE INNOVATION

CENTRE FOR SOCIAL INNOVATION

stärkere Differenzierung der Instrumente in Bezug auf die Gegebenheiten der unterschiedlichen Standorte als prinzipieller Erfolgsfaktor hervorzuheben. Die hohe Autonomie in der Personalplanung und Leistungsgestaltung vor Ort führt jedoch auch zu einer starken Abhängigkeit der Aktivitätsportfolios und der Qualität der Leistungen vom Einsatz der "richtigen Leute". Dies wird gerade auch in Richtung der WissenschaftsrätInnen adressiert, die sich oftmals mit (zu) hohen Erwartungen im Verhältnis zu ihren Ressourcen konfrontiert sehen würden. Hier lassen sich, laut Evaluierung, große Unterschiede in den Aktivitätsniveaus beobachten.

Das Prinzip der mehrjährigen Leistungsvereinbarungen mit individuellen Zielsetzungen für die einzelnen Standorte als strategischer Entwicklungsrahmen wird in Verbindung mit den jährlich vereinbarten Umsetzungs-Prioritäten als erfolgreiches Steuerungsinstrument gesehen. Gleichwohl wird Verbesserungsbedarf in der mittelfristigen standortspezifischen Strategieentwicklung gefordert, insbesondere in Verbindung mit einer stärkeren Priorisierung der Leistungs-Portfolios („,weniger ist mehr")

Als persistente Herausforderung, trotz Leistungsvereinbarungen und verhältnismäßig konzisem Wirkverständnis (siehe dazu Kapitel 6), wird die Entwicklung einer sinnvollen und aussagekräftigen wirkungsorientierten Indikatorik gesehen.

Von zentraler Bedeutung ist der Austausch innerhalb des Netzwerkes. Dieser erfolgt sowohl auf organisatorischer Ebene zwischen den Standorten aber auch zwischen den Kundlnnen und Stakeholdern. Dies erlaubt den Austausch von Erfahrungen ebenso wie die Bildung weiterer Netzwerke.

Die Vertretung zentraler Schweizer BFI-Akteure im swissnex-Committee trägt zudem zu einer breiten Verankerung und einem hohen Commitment der swissnex in der Schweizer BFI-Landschaft bei und erlaubt zudem eine bedarfsorientierte Weiterentwicklung des Modells. Dieser direkte Austausch und die enge Vernetzung der Akteure mag mit ein Grund sein, warum in der Evaluierung traditionelle Kommunikationsinstrumente wie Newsletter, Jahresberichte etc. infrage gestellt werden. 


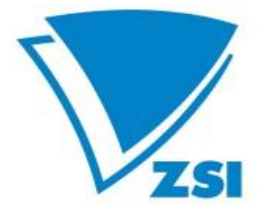

\section{Wirkungsmodelle entlang einer Output - Outcome - Impact Logik}

Die Entwicklung eines Wirkmodells ist von der Verfügbarkeit klarer Zielsetzungen sowie Vorstellungen über das jeweilige Aktivitätsportfolio der beiden OSTA abhängig. In diesem Zusammenhang ist festzustellen, dass die aktuelle interministerielle Rahmenvereinbarung aus dem Jahr 2016, wie sie dem Evaluierungsteam vorliegt, in Bezug auf eben diesen Anspruch sehr allgemein gefasst und auch wenig spezifisch in Bezug auf die Anforderungen an FTI-Politik in Nordamerika bzw. China ist.

Die Erarbeitung eines Wirkmodells für OSTA sowie entsprechender Wirkungsdimensionen bedarf daher jedenfalls einer stärker abgestimmten Klärung des strategischen Auftrages sowie der Positionierung im jeweiligen Sitzland der beiden OSTA durch die verantwortlichen Ministerien. Dies beinhaltet die Festlegung klarer strategischer Leitlinien als Kontext für die jeweiligen Einzelmaßnahmen und entsprechend mittelfristige Arbeitspläne für die beiden OSTA.

Ausgehend von diesen grundsätzlichen Anforderungen wird die Entwicklung spezifischer Wirkungsmodelle entlang der gängigen Output - Outcome - Impact-Logik (O-O-I) vorgeschlagen:

- Output: messbare, unmittelbare Produkte bzw. unmittelbaren Resultate der Aktivitäten

- Outcome: (mittelfristige) Nutzen/Effekte der Aktivitäten auf unmittelbare Zielgruppe

- Impact: Intendierte (evtl. auch nicht-intendierte) Wirkungen der Intervention auch über die intendierte Zielgruppe hinaus.

Die jeweilige Indikatoren-Entwicklung wiederum sollte dabei den sog. ,SMART'-Kriterien entsprechen:

- Spezifisch (in Bezug auf die jeweilige Zielsetzung),

- Messbar, i.e. Veränderungen können qualitative beschrieben werden und sind idealerweise auch zählbar,

- Attraktiv im Sinne von „erzielbar“ (,attainable'), d.h. in Bezug auf die entsprechenden Zielsetzungen müssen die entsprechenden Indikatoren akzeptiert sein,

- Relevant, d.h. es muss ein klarer Zusammenhang zwischen der Indikatorentwicklung und den theoretischen Überlegungen zu seiner Entwicklung in Bezug auf die jeweilige Zielsetzung herstellbar sein,

- Terminisierbar, d.h. klare Fristigkeiten und Regelmäßigkeit in der Verfügbarkeit. 


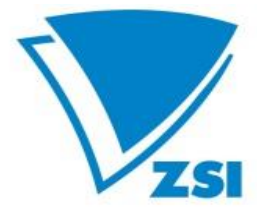

ZENTRUM FÜR SOZIALE INNOVATION

CENTRE FOR SOCIAL INNOVATION

Vorbild hierfür kann die Schweiz sein, wie nachfolgende Darstellung des Wirkmodells zu den swissnex zeigt (siehe Abbildung 4). Schon jetzt sind auf Basis der OSTA-Jahresberichte Output- und auch Outcome-Indikatoren ableitbar aber tlw. schwer aufzufinden.

Abbildung 4: swissnex Wirkmodell

\begin{tabular}{|c|c|c|c|c|}
\hline ZIEL UND MISSION & VOLLZUG & OUTPUT & IMPACT (Wirkung) & OUTCOME \\
\hline $\begin{array}{l}\text { Unterstützung der } \\
\text { Internationalisierung } \\
\text { schweizerischer } \\
\text { BFI-Akteure und } \\
\text { Promotion des } \\
\text { BFI-Standortes } \\
\text { Schweiz durch: } \\
\text { - Vernetzung } \\
\text { - Erzeugung von } \\
\text { Visibilität } \\
\text { - Informationen und } \\
\text { Beratungen } \\
\text { - Inspiration und } \\
\text { Förderung von } \\
\text { Wissensaustausch }\end{array}$ & $\begin{array}{l}\text { swissnex Modell als } \\
\text { Plattform basierend } \\
\text { auf: } \\
\text { - Autonomie } \\
\text { - Unternehmertum } \\
\text { - Standortwahl } \\
\text { - Partnerschaftliches } \\
\text { Finanzierungsmo- } \\
\text { dell } \\
\text { Vollzug ist nur durch } \\
\text { Kooperation mit } \\
\text { Partnerorganisatio- } \\
\text { nen möglich }\end{array}$ & $\begin{array}{l}\text { - Events (Panels, } \\
\text { Roundtables, etc.) } \\
\text { - } 1 \text { on } 1 \text { Meetings, } \\
\text { Beratungsge- } \\
\text { spräche, virtuelle } \\
\text { Vernetzung } \\
\text { - Delegationsvisiten/ } \\
\text { Study Tours } \\
\text { - Social Media-Prä- } \\
\text { senz, Newsletters, } \\
\text { Öffentlichkeitsar- } \\
\text { beit } \\
\text { - Information, Bera- } \\
\text { tung und Scouting }\end{array}$ & $\begin{array}{l}\text { Individuell: } \\
\text { Vernetzte Forscher, } \\
\text { Jungunternehmer \& } \\
\text { Policymakers, erfolg- } \\
\text { reiches Internatio- } \\
\text { nalisierungs-Projekt, } \\
\text { Visibilität, Inspiration, } \\
\text { Know-how } \\
\text { Institutionell: } \\
\text { Institutionelle Part- } \\
\text { nerschaften, allge- } \\
\text { meine Visibilität der } \\
\text { Institution sowie des } \\
\text { BFI-Platzes Schweiz } \\
\text { im Ausland }\end{array}$ & $\begin{array}{l}\text { Schweiz wird im } \\
\text { Ausland als global } \\
\text { führender BFI-Stand- } \\
\text { ort wahrgenommen. } \\
\text { BFI-Landschaft } \\
\text { Schweiz ist interna- } \\
\text { tional vernetzt und } \\
\text { sichtbar. } \\
\text { Schweizer BFI- } \\
\text { Akteure sind über } \\
\text { neuste globale } \\
\text { Trends und Oppor- } \\
\text { tunitäten informiert. }\end{array}$ \\
\hline
\end{tabular}

Quelle: SBFI (2015).

Sowohl die jährlichen Arbeitsprogramme als auch die entsprechenden Jahresberichte erlauben die Ableitung folgender zentraler strategischer Zieldimensionen für die OSTA vonseiten der auftraggebenden Ressorts:

- FTI-Politikunterstützung, Analysen, Aufzeigen von Trends

- Unterstützung bilateraler Kooperationen (auch in Verbindung mit nationalen und internationalen Programmen)

- Unterstützung des Technologie-, Wissens- und Know-how-Transfers

- Betreuung österreichischer Unternehmen, Forschungseinrichtungen und Forschenden

- Betreuung von Forschungseinrichtungen und Forschenden im Sitzland mit Interesse an Österreich

- Branding des österreichischen FTI-Standortes (Öffentlichkeitsarbeit)

- Ausbau EU und multilaterale Kooperationen 


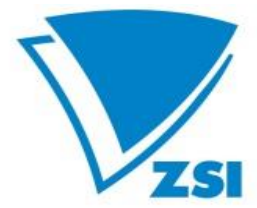

ZENTRUM FÜR SOZIALE INNOVATION

CENTRE FOR SOCIAL INNOVATION

Diese abgeleiteten strategischen Zieldimensionen bildeten die Grundlage für die Entwicklung eines theoretischen Wirkmodells inkl. entsprechender Indikatorik, nach den eingangs dargestellten Kriterien. Die Kalibrierung eines solchen Modells ist jedoch in weiterer Folge Sache der verantwortlichen Ministerien. Das vollständige Modell ist in Annex 10.2.

Nachfolgend ist ein entsprechender Bestandteil des Wirkmodells zur Zielsetzung „Unterstützung des Technologie- Wissens- und Know-how-Transfers" beispielhaft dargestellt.

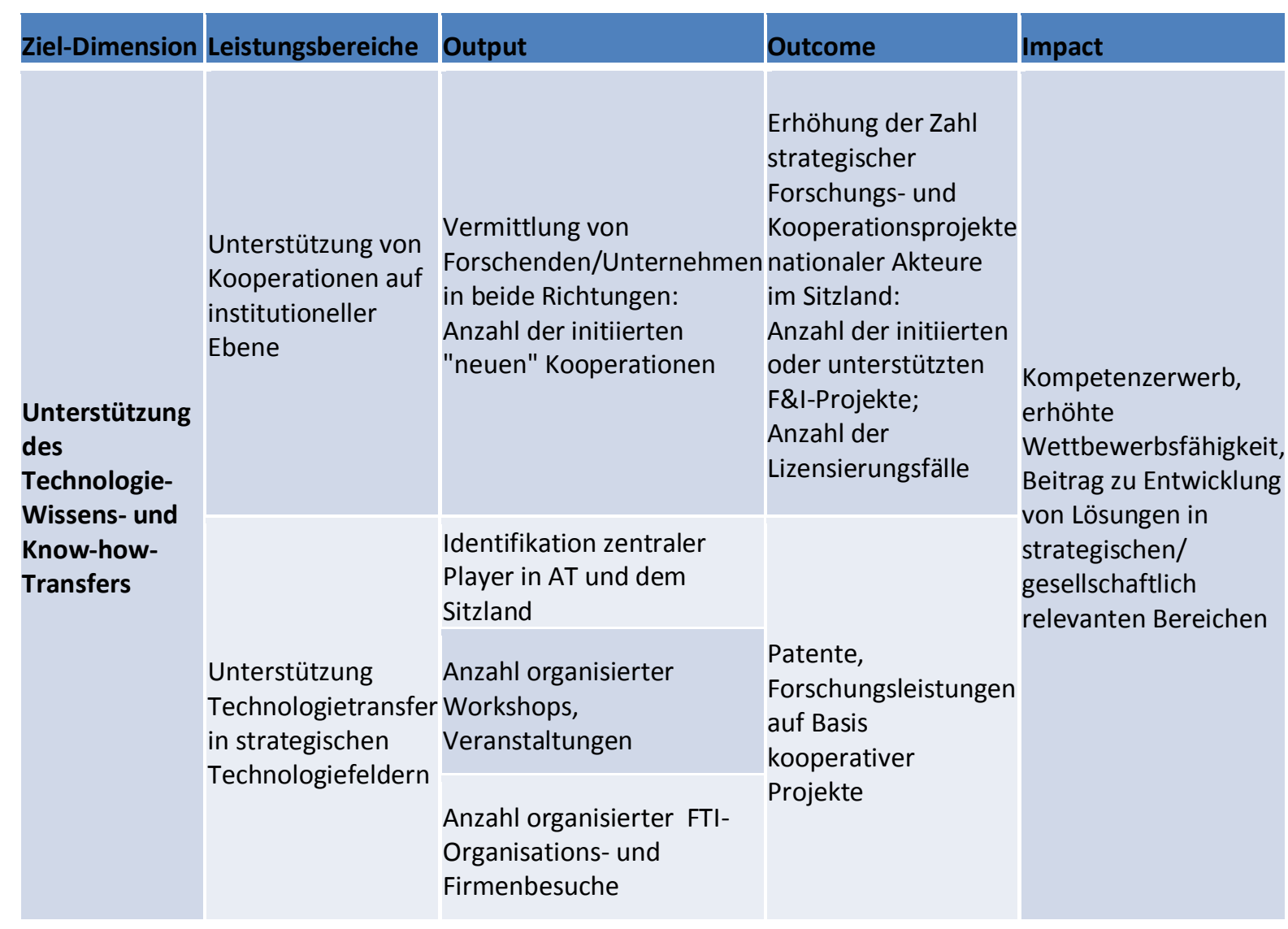

Quelle: Eigene Darstellung. 


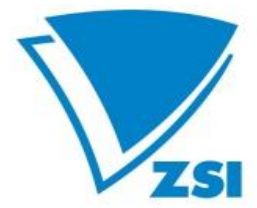

ZENTRUM FÜR SOZIALE INNOVATION

CENTRE FOR SOCIAL INNOVATION

\section{OSTA Washington}

OSTA Washington wurde bereits im Jahr 1999 gegründet und blickt damit auf eine zwanzigjährige überwiegend erfolgreiche - Geschichte zurück. Für österreichische Stakeholder stellt die sichtbarste und bestbesuchte Aktivität das jährliche ARIT dar. In Nordamerika wird vor allem die Beratung und Betreuung der österreichischen Forschenden vor Ort wahrgenommen und geschätzt.

Stand zunächst die Umkehrung des „Brain Drains“ nach Nordamerika im Vordergrund, fokussierte sich OSTA Washington in der Folge auf die Vernetzung österreichischer Forschenden in Nordamerika. Wenngleich inzwischen weitere Aktivitäten hinzu gekommen sind (siehe für einen Überblick 7.1 sowie 4.3) zieht das BMVIT aus der Konzentration auf die Betreuung von Forschenden die Konsequenz, dass es sich nicht sich nicht ausreichend mit seinen Themen und vor allem bei der Unterstützung seiner Kernaufgaben repräsentiert sieht. Dazu eine Person aus dem Ressort:

- „Dieser Fokus auf die Vernetzung von Wissenschaftlern (Betreuung von Wissenschaftlern vor Ort bzw. Organisation des ARIT) nimmt den überwiegenden Anteil der Arbeits- und Geldressourcen des OSTA Washington in Anspruch. Und auch wenn das BMVIT den Wert eines Wissenschaftler-Netzwerks und dessen Betreuung per se anerkennt, so ist es doch keine Aufgabe des BMVIT Wissenschaftler zu betreuen."

\subsection{Zentrale Aktivitäten}

Die Aktivitäten des OSTA Washington gliedern sich in vier zentrale Aufgabenbereiche, die auch in den Arbeitsprogrammen und Jahresberichten entsprechend genannt sind:

\section{Dienstleistungen für RINA Mitglieder}

Nach dem Leitsatz "Inform - Assist - Connect" unterstützt das OSTA Team RINA-Mitglieder in allen Aspekten ihrer Karriereentwicklung während des Aufenthalts in Nordamerika. Die Nutzung des Netzwerks stützt sich auf persönliche Kontakte der Mitglieder untereinander sowie auf den Kontakten, die individuelle OSTA Teammitglieder mit den RINA Mitgliedern halten. Insgesamt werden Anfragen sehr individuell und persönlich beantwortet. Neben Einladungen und Informationen zu Veranstaltungen werden RINA Mitglieder auch zU Immigration und VisaAngelegenheiten, zu Förderungsmöglichkeiten, zu Doppelstaatsbürgerschaftsanträgen und Kooperationsmöglichkeiten beraten. Siehe hierzu genauer Abschnitt 5.5

\section{Networking Veranstaltungen und Besuchsprogramme}




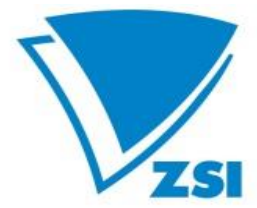

ZENTRUM FÜR SOZIALE INNOVATION

CENTRE FOR SOCIAL INNOVATION

Neben dem jährlichen ARIT (siehe 5.4) organisiert und unterstützt OSTA Washington eine ganze Reihe weiterer Veranstaltungen und Netzwerktreffen und präsentiert OSTA bzw. die österreichische Community auf Messen, Konferenzen und Diskussionen. Im Jahr 2018 waren dies:

- Die jährliche Netzwerkkonferenz der European Scientific Diaspora gemeinsam mit dem EURAXESS Mentoring Programm

- Das AAAS (American Association for the Advancement of Science) Annual Meeting im EUHouse

- Koordination des österreichischen Beitrags für SXSW in Texas (Einbindung der MedUni Wien sowie AIT)

- Austria to Austin (Startup-Austauschprogramm für Studierende, eine Initiative der US Botschaft in Österreich)

- Teilnahme an ALPs („Austrian Leadership Programs“, des Bundesministeriums für Europa, Integration und Äußeres in Kooperation mit der Industriellenvereinigung und der Wirtschaftskammer Österreich)

- Teilnahme an der Marshall Plan Foundation Reise

- Teilnahme an NAFSA (Association of International Educators) gemeinsam mit der OeAD

- Unterstützung der Wiener Wirtschaftsagentur (Erstellung eines Besuchsprogramms, Vernetzung mit Partnern in den Themen Smart Cities, Entrepreneurship, Business Incubation, Culinary Innovation)

- Besuchsreise und Vortrag des Wissenschafters / der Wissenschafterin des Jahres

- Gemeinsames Sommerfest mit Open Austria, Marshall Plan Foundation, Generalkonsulat Los Angeles, Wirtschaftskammer in Palo Alto

Darüber hinaus finden auch regelmäßige Netzwerktreffen mit ASciNA statt. Im Jahr 2019 sind ähnliche Aktivitäten durchgeführt worden bzw. in Planung.

\section{Politikberatung, Berichte und Analysen}

Jenseits der Arbeitsprogramme und Jahresberichte verfasst das OSTA Washington regelmäßig Berichte zu seinen Aktivitäten sowie Dossiers zu aktuellen Themen wie z.B. Cyber Security, Artificial Intelligence, Block Chain, Health oder Science Diplomacy. Diese Dossiers sind sorgfältig recherchiert und werden entsprechend geschätzt. Zentrale Stakeholder (Teilnehmende der Fokusgruppe) wünschen sich hier eine bessere Verbreitung. 


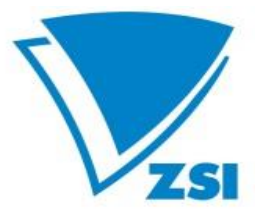

ZENTRUM FÜR SOZIALE INNOVATION

CENTRE FOR SOCIAL INNOVATION

Im Jahr 2018 wurde eine Studie zu Karrierechancen und zur Unterstützung der FTI Community durch OSTA durchgeführt, die auch eine Befragung aller RINA Mitglieder inkludierte.

In der Öffentlichkeitsarbeit kommuniziert OSTA Washington über das Netzwerk RINA, über den Blog BRIDGES sowie über Social Media (Twitter, Facebook und LinkedIn).

\subsection{Arbeitsprogramm/Jahresbericht}

Jedes Jahr wird dem Lenkungsausschuss ein Arbeitsprogramm inklusive Voranschlag vorgelegt. Der korrespondierende Jahresbericht zeigt auf, in welchem Umfang das beschlossene Programm umgesetzt werden konnte. Arbeitsprogramm und Jahresbericht sind klar gegliedert und sehr übersichtlich strukturiert. Der Stand der Umsetzung ist mit dieser Struktur gut dokumentiert. Die mangelnde Umsetzung einzelner Vorhaben wird transparent dargestellt und begründet. Projekte und Aktivitäten, die nicht im Arbeitsprogramm standen, aber dennoch umgesetzt wurden, werden als solche gekennzeichnet. Der Lenkungsausschuss kann sich auf Basis dieser Unterlagen ein sehr gutes Bild machen und ist ausreichend informiert.

Kritisch ist möglicherweise die Länge der Programme und Berichte zu sehen. Die Einführung von Mehrjahresprogrammen mit einer längerfristigeren und damit auch strategischeren Orientierung in Kombination mit sehr kurzen jährlichen Arbeitsprogrammen böte hier möglicherweise Potenzial für Verbesserungen. Mehrjahresprogramme sollten so gestaltet sein, dass hinreichend Freiraum bleibt, um kurzfristigen Bedarfen seitens der Ressorts oder der Stakeholder nachzukommen.

\subsection{ARIT}

ARIT (Austrian Research \& Innovation Talk) hat eine inzwischen recht lange Tradition: Im Jahr 2004 begann das BMVIT gemeinsam mit der WKO mit jährlichen „Austrian Science Talks“ (AST), im gleichen Jahr wurde auch das Programm „Brain Power“ initiiert, das bei der Vermittlung von Stellen zwischen Österreich und Nordamerika unterstützte.

Die jährlichen Netzwerktreffen wurden über die Jahre hinweg größtenteils vom BMVIT alleine bezahlt (mit Ausnahme des BMBWF-Empfangs zur ASciNA Awards Verleihung). Erst in den letzten Jahren wurde die Veranstaltung zu je einem Drittel von den drei die Grundkosten des OSTA bezahlenden Ressorts bezahlt. 


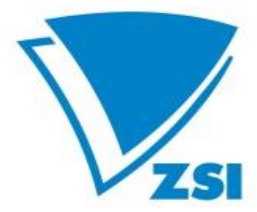

ZENTRUM FÜR SOZIALE INNOVATION

CENTRE FOR SOCIAL INNOVATION

ARIT wird insgesamt

- $\quad$ sehr breit kommuniziert (über RINA und BRIDGES, über Twitter, über österreichische Stellen) und wahrgenommen (war nahezu allen Befragten bekannt, viele Teilnehmende, Berichterstattung in Ö Medien)

- als "jährlicher Fix- und Höhepunkt" wahrgenommen

- sowohl von den interviewten Personen aus den Ressorts, als auch von den Befragten des online Surveys und den Teilnehmenden der Fokusgruppe überwiegend positiv bewertet (Austauschplattform, neue Trends und Entwicklungen, Networking).

Die letzten drei ARIT fanden in Toronto, Austin und Washington statt, die Anzahl der Teilnehmenden stieg von Jahr zu Jahr an.

ARIT 2016 Toronto: 122 Teilnehmende, Empfang (Welcome \& Networking Reception), Konferenz, Stakeholder Pitching, Poster Session und ASciNA Award

ARIT 2017 Austin: 131 Teilnehmende, Programm ähnlich wie in Toronto

ARIT 2018 Washington D.C.: 196 Teilnehmende, Programm ähnlich, zusätzlich EURAXESS Conference Allerdings fließen auch sehr viel Ressourcen in ARIT; sowohl Personalressourcen als auch Geld. Zielsetzung sowie Zielpublikum wurde von einigen der interviewten Ressorts als unklar bezeichnet, kritisiert wurde auch die jedes Jahr ähnliche Zusammensetzung der Teilnehmenden aus Österreich. Das BMVIT sieht seine Interessen im Kontext von ARIT nicht hinreichend abgebildet, woraus zunächst ein Rückzug aus der Finanzierung des ARIT resultierte. ARIT 2019 in New York wird aufgrund dieser Ressourcenbeschränkung kleiner werden als die Jahre zuvor. Es findet back to back mit Austria Connect statt, daher wurde der Standort New York gewählt.

\subsection{Stakeholder}

Aus Perspektive der im online-Survey befragten Personen sind die wichtigsten Leistungsbereiche des OSTA Washington:

- $\quad$ Beratung und Betreuung österreichischer Forschenden vor Ort

- Unterstützung der internationalen Mobilität von Forschenden 


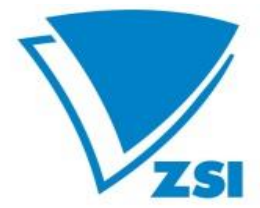

ZENTRUM FÜR SOZIALE INNOVATION

CENTRE FOR SOCIAL ININOVATION

Ebenfalls wichtig wird die Herstellung von Kontakten, die Unterstützung bi- und multilateraler Kooperationen sowie die Daten- und Informationsbereitstellung zu den USA angesehen.

Die Frage, welche Aufgaben künftig stärker wahrgenommen werden sollen, ergibt ein ähnliches Bild.

Abbildung 5: Aufgaben, die OSTA Washington künftig stärker wahrnehmen soll

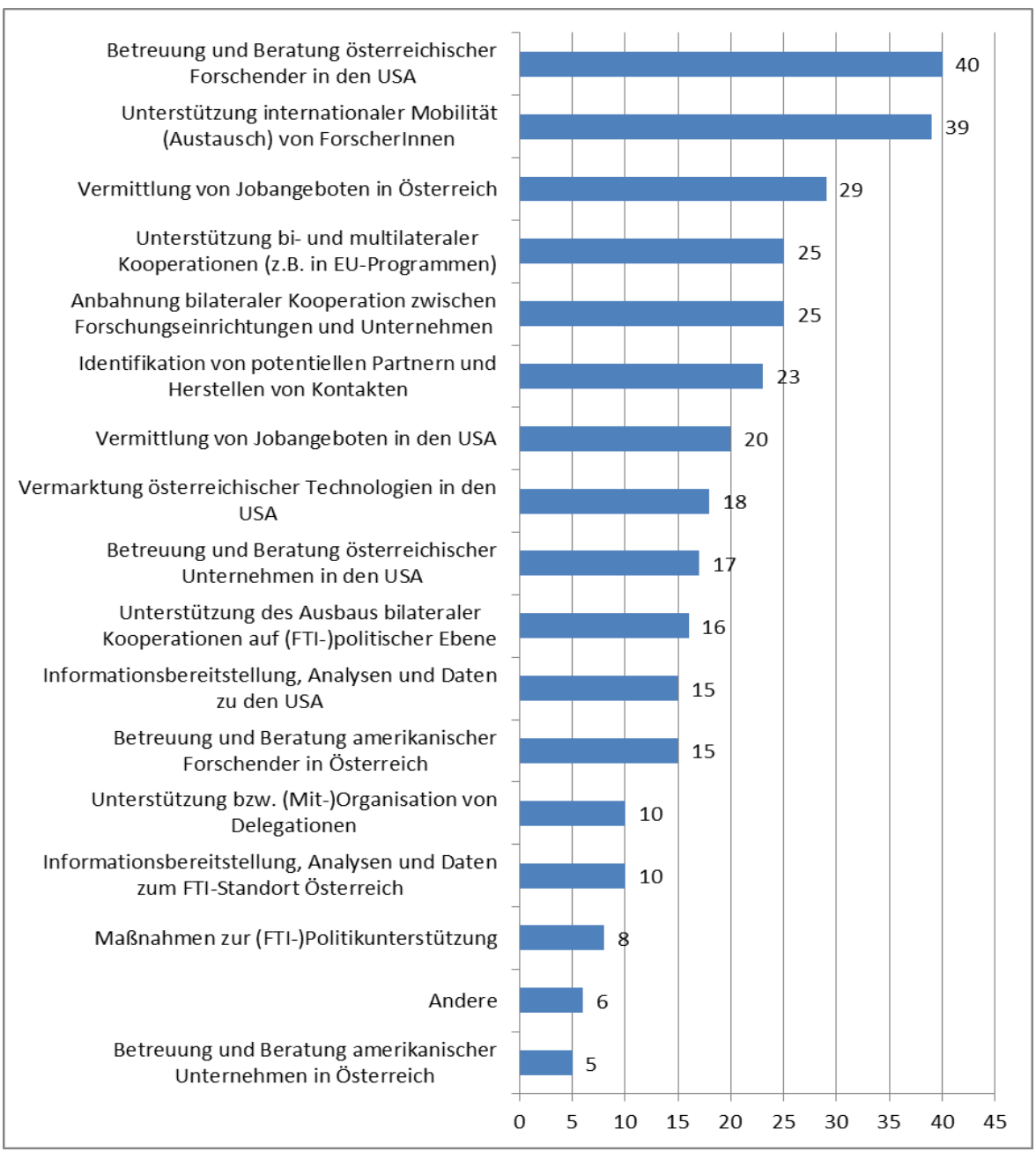

Quelle: JOANNEUM RESEARCH

Die Zufriedenheit der Befragten ist mehrheitlich hoch. Dies deckt sich auch mit den vonseiten den Teilnehmenden der Fokusgruppe (siehe die Auflistung der Teilnehmenden im Anhang) geäußerten Meinungen. Auch dort zeigt sich, dass OSTA Washington vor allem aufgrund der Veranstaltungen und Netzwerktreffen (in erster Linie ARIT) bekannt und präsent ist. Auch ASciNA spielt in der Wahrnehmung der österreichischen Stakeholder eine wichtige Rolle. 


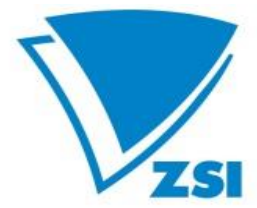

ZENTRUM FÜR SOZIALE INNOVATION

CENTRE FOR SOCIAL INNOVATION

Ebenfalls zeigt sich ein überwiegend positives Bild zur Bedeutung des OSTA Washington für die österreichische Forschung-Community. Allerdings erklärten die anwesenden Forschungseinrichtungen und Agenturen, dass sie ihre eigenen Netzwerke hätten und daher nur wenig auf die Vermittlungsleistungen des OSTA zurückgriffen. Von einem Anwesenden wurde zusätzlich „Fundraining" als interessantes künftiges Betätigungsfeld für OSTA genannt. Ein weiterer Diskussionspunkt betraf die Konzentration auf eher wissenschaftliche Themen: Wenngleich diese Konzentration die vorrangige Wahrnehmung der Teilnehmenden widerspiegelte, so wurde doch von einigen betont, dass OSTA sich auch in der Start-up Szene sehr gut auskenne und Firmenkontakte vermittele.

Explizit positiv wurde genannt:

- „Beim OSTA Washington kann man einfach anrufen und bekommt Service“

- „OSTA hilft gezielt bei der Suche nach Kooperationspartnern, leitet Anfragen weiter, ist kooperativ und serviceorientiert."

- „OSTA hat seine Leistungen pro-aktiv vorgestellt, wir nutzen ARIT für Recruiting und arbeiten auch inhaltlich in einigen Bereichen zusammen (z.B. Al, personalised medicine, social sciences)“

- „Es gibt eine gute Zusammenarbeit der AußenwirtschaftsCenter mit OSTA, weil es eine gute Arbeitsteilung mit wenig Überschneidung gibt. Kooperation gibt es dort, wo über Förderprogramme insbesondere an der Science-Industry-Schnittstelle informiert wird, um Kooperationen einzufädeln."

Kritisch angemerkt wurde,

- dass es bislang wenig Bewusstsein hinsichtlich der Aktivitäten von OSTA Washington über die USA hinausgäbe,

- dass österreichische Unternehmen sich nur wenig angesprochen sähen,

- dass es eine zu starke Konzentration auf die individuellen Forschenden und zu wenig institutionelle Kooperationen mit Universitäten gäbe (gleichzeitig wurde aber auch hinterfragt, ob die Universitäten eine solche Kooperation überhaupt bräuchten)

- dass die Rückholung österreichischer Forschenden aus den USA "nicht Aufgabe des OSTA“ sein solle. 


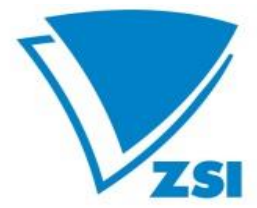

ZENTRUM FÜR SOZIALE INNOVATION

CENTRE FOR SOCIAL INNOVATION

Auch wurde vonseiten der Stakeholder der Wunsch geäußert, bei der Verbreitung von Dossiers und Berichten besser berücksichtigt zu werden, da dort oft interessante Themen angesprochen sind, die Informationen aber nur an die Ressorts gingen.

\subsection{RINA}

Bei RINA (Research and Innovation Network Austria) handelt es sich um ein sehr umfangreiches und gut gepflegtes Netzwerk, das von OSTA Washington betrieben wird. Das RINA setzt sich per April 2019 wie folgt zusammen (gesamt 2,960 aktive Kontakte gelistet, archivierte Kontakte sind zusätzlich noch 297 Personen):

- $\quad$ 965x ID 1: Austrian Researchers Currently in North America

- $\quad 1.435 \times$ ID 2: Austrian Researchers \& Innovators Formerly in North America (Alumni)

- 251x ID 3: Researchers with close ties to Austria and North America

- $\quad$ 146x ID 12: Austrian Innovators \& Entrepreneurs Currently in North America

- 163x ID 13: Austrian Grantees Currently in North America

Damit liegt der Schwerpunkt auf Forschenden, die entweder derzeit oder in der Vergangenheit in Nordamerika tätig sind/waren.

\section{Jahresvergleich der gelisteten RINA Kontakte}

Statistiken zur RINA Netzwerkentwicklung werden seit 2003 jährlich im Rahmen des „Annual Data Update" am OSTA Washington erfasst. Von 2003 bis 2019 stiegen die Mitgliedszahlen für RINA Mitglieder im Jahresdurchschnitt um 11.40\%.

Die nachstehende Auflistung zeigt die Entwicklung der Zahlen der „aktiven" RINA Mitglieder bis dato. Per Mai 2019 wurden 297 RINA Mitglieder aufgrund deren Ableben oder Verlassen des FTIBereichs archiviert. 


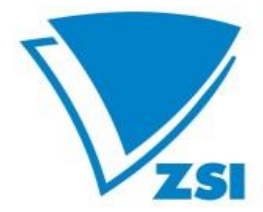

ZENTRUM FÜR SOZIALE INNOVATION

CENTRE FOR SOCIAL INNOVATION

Abbildung 6: Entwicklung der RINA Mitglieder

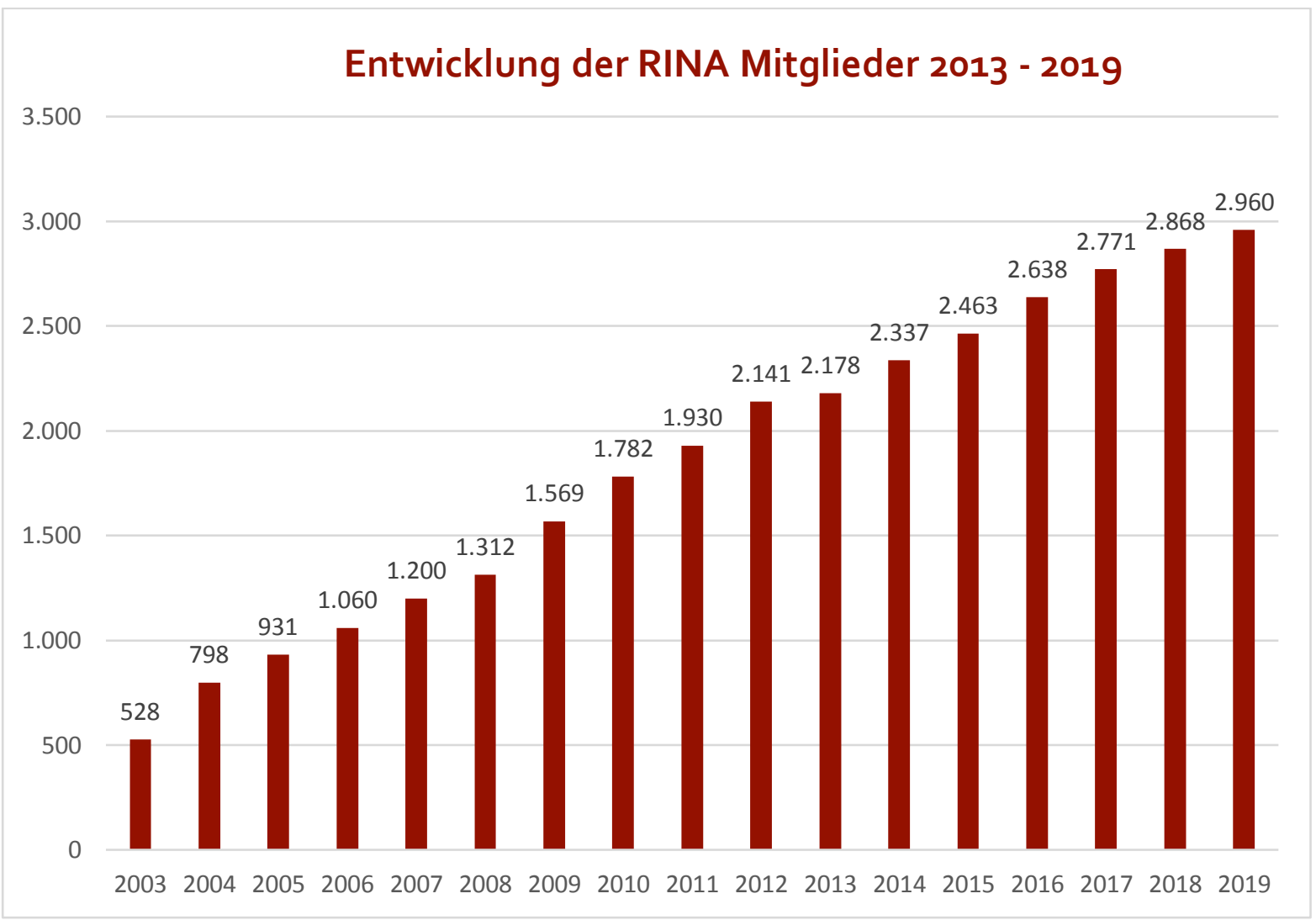

Quelle: OSTA Washington

\section{Nutzung des Netzwerks}

Nach dem Leitsatz "Inform - Assist - Connect" unterstützt das OSTA Team RINA Mitglieder in allen Aspekten ihrer Karriereentwicklung während des Aufenthalts in Nordamerika. Die Nutzung des Netzwerks stützt sich auf persönliche Kontakte der Mitglieder untereinander sowie auf dem Kontakt den individuelle OSTA Teammitglieder mit den RINA Mitgliedern halten.

RINA Mitglieder können einander informieren aber auch Informationen via dem OSTA Team beziehen. Sie können Assistenz und Kooperation anbieten aber auch Unterstützung von OSTA Seite erhalten. Sie können sich aber auch untereinander vernetzen und mit Hilfe des OSTA Teams mit FTI Stakeholdern in Österreich und Nordamerika in Kontakt treten.

\section{Informieren zu:}

- Karriere- und Weiterbildungsmöglichkeiten

- Forschungsförderungs- und Finanzierungsmöglichkeiten 
ZENTRUM FÜR SOZIALE INNOVATION

CENTRE FOR SOCIAL INNOVATION

- Neuerungen aus dem FTI Bereich

- Doppelbesteuerung

- Beibehaltung der Staatsbürgerschaft

\section{Assistieren bei:}

- Kooperationsanbahnung

- Karriereentwicklung

- Studierenden und Fakultätsaustausch

- Ankunft aus Österreich und Rückkehr nach Österreich

- Publikationen von wissenschaftlichen Leistungen

- Auszeichnungen und Ehrungen für wissenschaftliche Leistungen

- Visa Angelegenheiten

- Konferenzteilnahme und Vermittlung von Expertinnen und Experten

\section{Vernetzen mit:}

- Fachexperten/innen und Kollegen/innen

- FTI Einrichtungen

- Forschungsförderungsagenturen

- Universitäten und Fachhochschulen

- Kooperationspartner/innen aus der Industrie

- Wissenschaftsjournalisten/innen

- Interessensvereinigungen

OSTA Washington nutzt u.a. folgende Werkzeuge um diese individualisierte Betreuung umzusetzen:

- Direktkontakt (persönliche Treffen, Emails, Telefonate)

- Massenmails

- OSTA Website

- Bridges Blog

- Social Media (LinkedIn, Twitter, Facebook)

- Webinare 


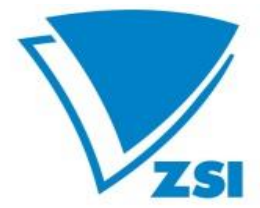

ZENTRUM FÜR SOZIALE INNOVATION

CENTRE FOR SOCIAL INNOVATION

- RINA LAB Podcast Reihe

- OSTA Datenbank

- Mentoring Programm

- Besuchsprogramme

- Veranstaltungen

- Skype

\section{Aussendungen 2018 an RINA Mitglieder}

Mit Hilfe der OSTA Datenbank werden Email Kampagnen spezifisch auf die jeweiligen RINA Zielgruppen zugeschnitten. Z.B. werden Ausschreibungen im Kartografie Bereich an Forschende der Kategorien „Natural Sciences" sowie „Technology \& Engineering" bzw. spezifisch jene die mit den Termini „geography, cartography, geoinformation, ..." gekennzeichnet wurden gesendet.

So kann das OSTA Team sicherstellen, dass nur für das jeweilige RINA Mitglied relevante Informationen versendet werden. Diese selektive Vorgehensweise resultiert darin, dass Inhalte die vom OSTA Team ausgesendet werden auch tatsächlich von den RINA Mitgliedern konsumiert / gelesen werden. Dies zeigt auch die 77\%igen „Open-Rate” bei OSTA Aussendungen an das RINA (Non-Profit/Government Durchschnitt in den USA liegt vergleichsweise bei 24\%).

Gesamt wurden im Jahr 201888 Email Kampagnen zu diversen Themenbereichen für RINA Mitglieder etabliert. In Ergänzung zu den meist detaillierteren Aussendungen wurden diverse Informationen auch via der Social Media Kanäle LinkedIn (156 Posts), Twitter (538 Posts) und Facebook (206 Posts) unter RINA Mitgliedern verteilt.

Die individuelle Kontaktherstellung und -aufnahme via der OSTA Teammitglieder und einzelner RINA Mitglieder kann nicht quantifiziert werden.

\section{Vermittelte Kontakte und beantwortete Anfragen 2018}

RINA Mitglieder stellen entweder selbst Anfragen ans OSTA Team, oder unterstützen das OSTA Team bei der Betreuung von FTI Stakeholdern aus Österreich oder Nordamerika.

Im Jahr 2018 wurden in etwa 120 Anfragen via Anfragenmanagement des OSTA Washington behandelt. Das Anfragenmanagement des OSTA Washington inkludiert Kontaktvermittlung, Informationen zu Doppelstaatsbürgerschaft, Suche nach Förder-, Kooperations-, oder Jobmöglichkeiten, inhaltlicher Austausch, Informationsverbreitung, Vortragstätigkeit, 


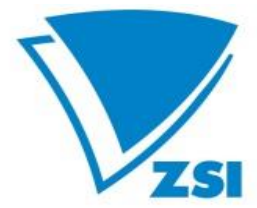

ZENTRUM FÜR SOZIALE INNOVATION

CENTRE FOR SOCIAL INNOVATION

Identifikation von Expertinnen und Experten, Visabestimmungen, etc.. Die Abwicklung einer "Anfrage" kann entweder bereits mit einem Email erledigt sein oder in einigen Fällen auch mehrere Monate oder mehrere Jahre davern und persönlichen Kontakt, Telefonate und Emails beinhalten.

\section{Direkte Interaktion der RINA Mitglieder untereinander}

RINA Mitglieder können bei OSTA Veranstaltungen und via der OSTA LinkedIn Plattform direkt miteinander in Kontakt treten und sich vernetzen.

Darüber hinaus fungieren OSTA Washington Teammitglieder derzeit aus Datenschutzgründen als „Vermittler" bei der direkten Vernetzung der RINA Mitglieder untereinander.

Zum Beispiel kontaktiert Forscherin A im Bereich Onkologie das OSTA Washington mit der Anregung, dass sie mit österreichischen Wissenschaftlerinnen und Wissenschaftlern für Projekt B kooperieren will. OSTA Washington erfasst erste Details und wählt - je nachdem wie spezifisch das Ansuchen ist - eine der folgenden Vorgehensweisen:

- OSTA sendet eine Nachricht an alle andere Onkologinnen und Onkologen im RINA aus mit der Bitte, sich bei Interesse direkt mit Forscherin A in Verbindung zu setzen.

- OSTA kontaktiert einige individuelle Onkologinnen und Onkologen im RINA via Email mit den Details und der Nachfrage, ob eine persönliche Vorstellung gewünscht sei.

- OSTA ruft vereinzelte Onkologinnen und Onkologen im RINA an, um deren Interesse an dem Projekt/der Kooperation abzuschätzen und um die Erlaubnis für eine persönliche Kontaktherstellung (via Email) einzuholen.

Aufgrund der persönlichen Betreuung (RINA Mitglieder kennen die OSTA Teammitglieder persönlich und vertrauen auf deren Einschätzung) lassen sich bei dieser Art der Kontaktvermittlung gute Ergebnisse erzielen.

\subsection{Governance vor Ort, Abstimmung mit anderen Akteuren}

\section{Governance}

Eingliederung in die Botschaft: Die OSTA sind gemäß der interministeriellen Rahmenvereinbarung "die für Wissenschaft, Forschung, Innovation und Technologie zuständigen Abteilungen an österreichischen Vertretungsbehörden und organisatorisch in diese eingegliedert". 


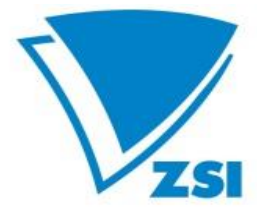

ZENTRUM FÜR SOZIALE INNOVATION

CENTRE FOR SOCIAL INNOVATION

Diese Eingliederung hat zur Konsequenz, dass der österreichische Botschafter als Dienstgeber der im OSTA beschäftigten Personen fungiert, ohne jedoch eine inhaltliche Leitungsfunktion inne zu haben. Diese Konstruktion der Trennung zwischen dienstrechtlicher und inhaltlicher Leitung bedarf eines guten Einvernehmens zwischen allen beteiligten Akteuren. Der österreichische Botschafter hatte vorgeschlagen, die genauen Verantwortungen der Vertretungsbehörde, der Leitung des OSTA sowie der inhaltlich verantwortlichen Ressorts in einer Geschäftsordnung zu definieren.

Leitung des OSTA Washington: Derzeit sieht die Rahmenvereinbarung vor, dass die Besetzung der Leitungsfunktion als direkte Entsendung des BMEIA gemäß einer „internen Ausschreibung für den höheren auswärtigen Dienst" (Interministerielle Rahmenvereinbarung S. 5) erfolgt. Alternativ dazu kann die Dienstzuteilung aus einem der anderen beteiligten Ressorts im Rahmen einer finanzierten Planstelle erfolgen. Da diese Alternative derzeit gemäß den Aussagen der Ressorts wenig Chance auf Realisierung hat, bleibt die Entsendung des BMEIA. Damit sind Personen, die nicht dem höheren auswärtigen Dienst angehören, möglicherweise aber die Qualifikation hätten, das OSTA zu leiten, ausgeschlossen. Dies bedeutet eine sehr kleine Gruppe an Personen, aus denen gewählt werden kann. Erschwerend kommt hinzu, dass die OSTA-Leitung keine attraktive Position für einen diplomatischen Karriereweg darstellt. Bei der letzten Ausschreibung für OSTA Washington gab es nur eine Bewerbung.

Zu dieser Konstruktion gab es sowohl in der Fokusgruppe als auch in den Interviews mit den Ressorts unterschiedliche Meinungsäußerungen: Der Eingliederung in die Botschaft wurde weitgehend zugestimmt: "wichtiger Türöffner", "grundsätzlich gute Konstruktion", "OSTA soll das offizielle Österreich sein", "Science Diplomacy ist eine wichtige und immer wichtiger werdende Aufgabe", allerdings gab es auch einige kritische Stimmen, die beispielsweise fanden, die "OSTA könnten sichtbarer werden, wenn sie nicht in der Botschaft versteckt wären".

Auch zu der Frage, ob die Leitung des OSTA dem höheren diplomatischen Dienst angehören sollte, gab es unterschiedliche Stimmen sowohl von Seiten der Ressorts als auch von Seiten der Stakeholder. Für die Konstruktion spricht, dass damit „Außenpolitik und FTI-Politik auf selbstverständlich Weise zusammenarbeiten". Auch wurde argumentiert, dass sich „Personen des höheren Auswärtigen Dienstes immer wieder schnell in neue Themen einarbeiten und sie tun dies auch erfolgreich". Dagegen spricht, dass der Pool geeigneter Personen ex-ante klein ist und die Anbindung an die zentralen Stakeholder und Institutionen in Österreich sich eher schwierig gestaltet, weil „er nicht aus der Szene kommt“. Sehr dezidiert äußerte eine interviewte Person: „Die Leitungspersonen verfügen nicht über die notwendige Qualifikation und Vernetzung mit den österreichischen Playern" 


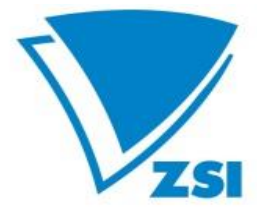

ZENTRUM FÜR SOZIALE INNOVATION

CENTRE FOR SOCIAL INNOVATION

\section{Abstimmung vor Ort:}

Die Kooperation mit AußenwirtschaftsCentern (AWC) werden von beiden Seiten (wie auch von der WKÖ in Österreich) als bereichernd und komplementär angesehen: „Kooperation gibt es insbesondere an der Science-Industry-Schnittstelle". Auch gibt es gemeinsame Initiativen (mit open Austria beispielsweise das gemeinsames Sommerfest im Silicon Valley) und gegenseitige operative Unterstützung (z.B. ARIT 2019 in New York).

- Bei den Interviews mit den Ressorts zeigten sich unterschiedliche Vorstellung zum Zusammenwirken zwischen OSTA Washington und den AWC: Einige Interviewpartner meinten, fehlende Aktivitäten des OSTA im Bereich Technologietransfer würden auch von den AWC nicht abgedeckt werden, andere sprachen sich für eine engere Zusammenarbeit aus, wiederum andere meinten, die Arbeit des OSTA sei im Unterschied zu den AWC wenig sichtbar und brächte wenig konkreten Output.

- Auch blieb die konkrete Zusammenarbeit mit „Open Austria“ und anderen Aktivitäten in der Bay Area eher unklar, ebenso wie dir Rolle von Clara Blume als Kontaktperson zu OSTA Washington im „Open Austria“ Team.

Die Zusammenarbeit mit ASciNA ist nicht ganz konfliktfrei. Auf beiden Seiten gibt es eine gewisse Furcht vor einer gegenseitigen Vereinnahmung. Da für den wissenschaftlichen Teil der Kunden und Kundinnen die beiden Marken „ASciNA“ und "OSTA“ nur schwer voneinander unterscheidbar sind, böte sich eine gute und enge Zusammenarbeit an, zumal ASciNA als privater Verein mit dezentraler Chapter-Struktur über keine administrativen Ressourcen verfügt. Im April 2019 wurde ein neuer Vorstand mit Dietrich Haubenberger als Präsident und Elisa Arthofer aus Washington D.C. als eine der stellvertretenden Personen gewählt. In dieser personellen Neuaufstellung liegt auch eine Chance, dass die Zusammenarbeit zwischen OSTA und ASciNA sich künftig wieder enger gestalten wird.

Gemeinsame Veranstaltungen und Aktivitäten gibt es weiterhin mit der OeAD, der Marshall Plan Foundation, dem AIT, LISA Vienna, INITS etc.

OSTA Washington arbeitet mit einigen amerikanischen Partnern sehr eng und sehr konstruktiv zusammen. Dazu zählen Fulbright, nature, die US Botschaft in Wien, Think Tanks wie z.B. die information technology and innovation foundation, aber auch Unternehmen wie z.B. Google). Es gibt gemeinsame Veranstaltungen und Initiativen wie Austria2Austin, Webinare, etc. Die Einschätzung 


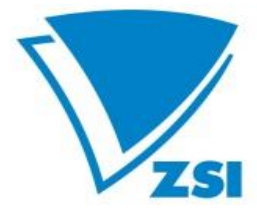

ZENTRUM FÜR SOZIALE INNOVATION

CENTRE FOR SOCIAL INNOVATION

dieser Partner ist überaus positiv: „it's Austria, the Netherlands and Switzerland which are active, we cooperate with them and are happy having OSTA as a contact point."

Im europäischen (EU) Kontext sind das AAAS (American Association for the Advancement of Science) Annual Meeting und EURAXESS wichtige Initiativen mit einem sichtbaren Beitrag des OSTA.

Einschätzung: OSTA Washington kooperiert mit einer Vielzahl an Partnern sowohl in Österreich als auch vor Ort, ist dort auch sichtbar, bekannt und wird geschätzt. Die Abstimmung und Arbeitsteilung mit den Akteuren vor Ort ist allerdings überwiegend ad-hoc, wenig systematisch und stark personengetrieben. Eine genauere Definition und längerfristige Ziele der Zusammenarbeit fehlen bislang.

\subsection{Verwaltung und Gebarung}

\section{Büroorganisation:}

Zum Zeitpunkt des Vor-Ort Besuches arbeiteten 5 Personen im OSTA Washington: Der Leiter Clemens Mantl, die stellvertretende Leiterin Simone Pötscher, der Projektmanager Robin Tim Weis, die Office Managerin Brittany Boykin und die temporär vom BMBWF entsandte Tanja Ölschläger.

Den Personen sind Themen wie auch zuständige Ressorts zugeordnet, was die Kommunikation mit den Ministerien wie auch den Stakeholdern erleichtert. Derzeit ist allerdings eine Position (BMVIT) vakant. Alle Mitarbeitenden sind sehr gut qualifiziert, hoch motiviert und initiativ, die Vernetzung vor Ort ist ausgezeichnet, die Anbindung an Stakeholder in Österreich könnte verstärkt werden (vor allem der Kontakt zu den Universitäten wie auch den zentralen FTI Programmen wie z.B. COMET, Beyond Europe, CDG, FWF Programme)

Mit der Geschäftseinteilung gibt es definierte und verschriftlichte Verantwortungsbereiche und Pflichten für jede einzelne Mitarbeitende. Die Tools welche für die Buchhaltung, Zeiterfassung und das Projektmanagement in Verwendung sind (Teamwork.com, Quickbooks) sind professionell und erlauben eine gut nachvollziehbare Büroorganisation.

\section{Gebarung:}

Das Büro funktioniert papierlos: Dokumente, Rechnungen und andere Unterlagen, die als Hardcopy vorliegen werden eingescannt und in der Folge geschreddert. Auf diese Weise erübrigt sich auch das Einschließen vertraulicher Dokumente. 


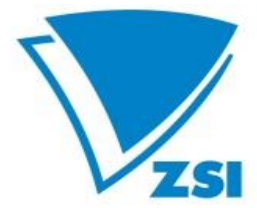

ZENTRUM FÜR SOZIALE INNOVATION

CENTRE FOR SOCIAL ININOVATION

Eine Handkassa existiert nicht, alle Zahlungen werden mit Schecks oder Kreditkarte getätigt, das Büro ist damit komplett bargeldlos. Es gibt genau definierte und in den Bürorichtlinien verschriftlichte Grenzen für die Ausgaben, welche die Mitarbeitenden tätigen können.

Die aktuellen Salden wie auch die Konsistenz der Einnahmen-Ausgaben Rechnung wurden überprüft (5 größte Ausgaben und Einnahmen im Jahr 2019 sowie 5 zufällig gewählte Ausgaben und Einnahmen). Hier sind keine Differenzen oder Unregelmäßigkeiten zu beanstanden.

Ebenso wurden die Inventarisierungen auf Basis einer Stichprobe von drei Einrichtungsgegenständen überprüft: Ein Tisch, ein MacBook und ein Stehpult waren ordnungsgemäß inventarisiert, die Inventarnummer auf dem Gegenstand stimmte mit Inventarverzeichnis überein.

\section{OSTA Peking}

\subsection{Zentrale Aktivitäten}

Ähnlich OSTA Washington sind die in Arbeitsprogramm und Jahresbericht aufgelisteten Aktivitäten sechs Zielen zugeordnet:

Ziel 1: Policy Support

Ziel 2: Bilaterale Kooperationen ausbauen

Ziel 3: Österreichische Forschungseinrichtungen und Forscher/innen betreuen

Ziel 4: Österreichische Technologien vermarkten

Ziel 5: EU und multilaterale Kooperationen ausbauen

Ziel 6: Öffentlichkeitsarbeit

Die wichtigsten Aktivitäten im Jahr 2018 waren:

- Werkstoffe-Delegationsreise in Vorbereitung auf den fünften Joint Call der FFG mit der CAS (Chinese Academy of Science)

- Betreuung Joint Calls

- Nachhaltige Vernetzung von Forschenden

- Technologientransfer und Technologievermarktung

- Outreach-Events China 


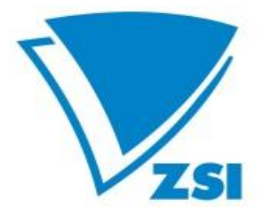

ZENTRUM FÜR SOZIALE INNOVATION

CENTRE FOR SOCIAL INNOVATION

Im Unterschied zu OSTA Washington stellt die Betreuung von Delegationsreisen einen deutlich wichtigeren Teil der Arbeit des OSTA Peking dar. Dies lässt sich vor allem mit den mangelnden Möglichkeiten der Durchführung eigenorganisierter Reisen vonseiten österreichischer Unternehmen, Forschungseinrichtungen oder auch Ressortvertretungen erklären. Eine weitere Besonderheit ist die Betreuung von Joint Calls (Kooperation mit CAS, Tecxport). Zu dieser im Portfolio von OSTA Peking zentralen Aktivität wurde vonseiten einiger Stakeholder kritisch angemerkt: „Bei der konkreten Antragsberatung ist fraglich, ob sie das in ausreichender Qualität können.“ (aus der Fokusgruppe) Im Gegenzug gibt es nur wenig Vernetzungsaktivitäten und Services für Forschende.

\subsection{Arbeitsprogramm/Jahresbericht}

Analog zu OSTA Washington weisen auch bei OSTA Peking das Arbeitsprogramm sowie der Jahresbericht eine sehr übersichtliche und einheitliche Gliederung auf:

Tabelle 1: Arbeitsprogramm und Jahresbericht OSTA Peking

\begin{tabular}{|l|l|}
\hline Arbeitsprogramm & Jahresbericht \\
\hline Aufgabenschwerpunkte & Höhepunkte des Jahres \\
\hline $\begin{array}{l}\text { Aufgaben im Detail (deutlich den 6 Zielen } \\
\text { zugeordnet, detaillierte Beschreibungen sehr } \\
\text { konkreter Vorhaben }\end{array}$ & $\begin{array}{l}\text { Aktivitäten und Maßnahmen (gegliedert nach } \\
\text { den 6 Zielen) }\end{array}$ \\
\hline Governance & Organisation \\
\hline Budget- und Finanzplanung & $\begin{array}{l}\text { Finanzielle Berichterstattung erfolgt im } \\
\text { Rahmen der Jahresabschlüsse }\end{array}$ \\
\hline
\end{tabular}

Aktuell sind die Berichte recht lang, der Jahresbericht umfasst etwa 25 Seiten; hinzu kommen Anhänge. Analog zu OSTA Washington sollte auch hier die Einführung von Mehrjahresprogrammen mit einer längerfristigeren und damit auch strategischeren Orientierung in Kombination mit kurzen jährlichen Arbeitsprogrammen überlegt werden. 


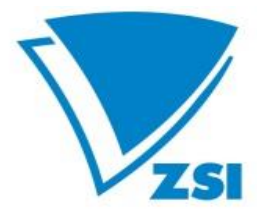

ZENTRUM FÜR SOZIALE INNOVATION

CENTRE FOR SOCIAL ININOVATION

Beide OSTA sollten hier einem einheitlichen Format folgen, das auch eine tabellarische Zusammenfassung auf Basis eines Template enthält.

\subsection{Informationsaufbereitung}

Die Übermittlung von Berichten erfolgt

- Im Rahmen der Jahresberichte

- Im Rahmen von Quartalsberichten

- Im Rahmen der Newsletters

OSTA Peking verfasst alle 1 bis 2 Monate einen Newsletter, um die österreichische Community zu Ereignissen in China zu informieren. Die Ressorts schätzen dies mehrheitlich; Stakeholder äußern sich hingegen kritisch und meinen, die Newsletter seien zu retrospektiv und ließen einen echten Neuigkeitscharakter vermissen.

Beim OSTA Peking laufen alle Berichte, die politisch (auch FTI-politisch) relevant sind, ausschließlich über die Botschaft ins BMEIA. Die Informationen müssen entweder übers BMEIA weiterverteilt oder informell übermittelt werden.

OSTA Peking ist nicht in Social Media aktiv (Ausnahme WeChat-Gruppe für Alumni unter OeADLeitung), die Homepage bietet eher wenig Informationen.

\subsection{Delegationsreisen}

Für den Zeitraum von September 2013 bis November 2018 liegen Informationen zu 21 Delegationsreisen vor. Die Reisen werden in erster Linie von BMVIT und FFG, aber auch von der WKÖ initiiert. Die Teilnehmenden kommen aus den folgenden Stakeholder Gruppen:

- Außeruniversitäre Forschungseinrichtungen (v.a. AIT, Joanneum Research),

- Universitäten (TU Wien, TU Graz, JKU, MUL)

- Unternehmen: Voest, AVL, AT\&S, etc., auch kleine, „nischenorientierte“ Unternehmen (z.B. akademische Spin-offs)

- $\quad$ Agenturen (hauptsächlich FFG)

- Ministerien (hauptsächlich BMVIT)

Bei den meisten Reisen nehmen 20-30 Personen teil. Wenn Minister oder Ministerinnen präsent sind, sind die Gruppen tendenziell größer ("politische Reisen"). 


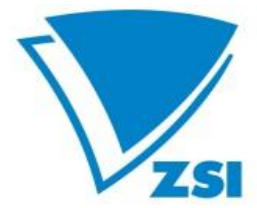

ZENTRUM FÜR SOZIALE INNOVATION

CENTRE FOR SOCIAL ININOVATION

Sehr üblich ist es, an Tagungen, Messen und sonstige Veranstaltungen mit einer Delegationsreise anzudocken. In einem solchen Kontext werden dann beispielsweise bilateralen Veranstaltungen organisiert (häufig Workshops mit Vorträgen durch österreichische und chinesische Forschende).

Auch dienen die Delegationsreisen der Unterstützung von bilateralen Kooperationen oder Projektverhandlungen. Auf chinesischer Seite sind die Ansprechpartner Universitäten, Forschungsinstitutionen, Regulierungsbehörden (z.B. im Verkehrssektor), Stadtverwaltungen (Smart Cities, Infrastruktur)

Thematisch sind die Reisen auf folgende Technologiebereiche fokussiert: Nanotechnologie, Werkstoffe, IKT, Umwelttechnik, Infrastrukturtechnologien (Smart Cities, Verkehr: Luftverkehr, Bahn) Abbildung 7: Delegationsreisen OSTA Peking nach Themen

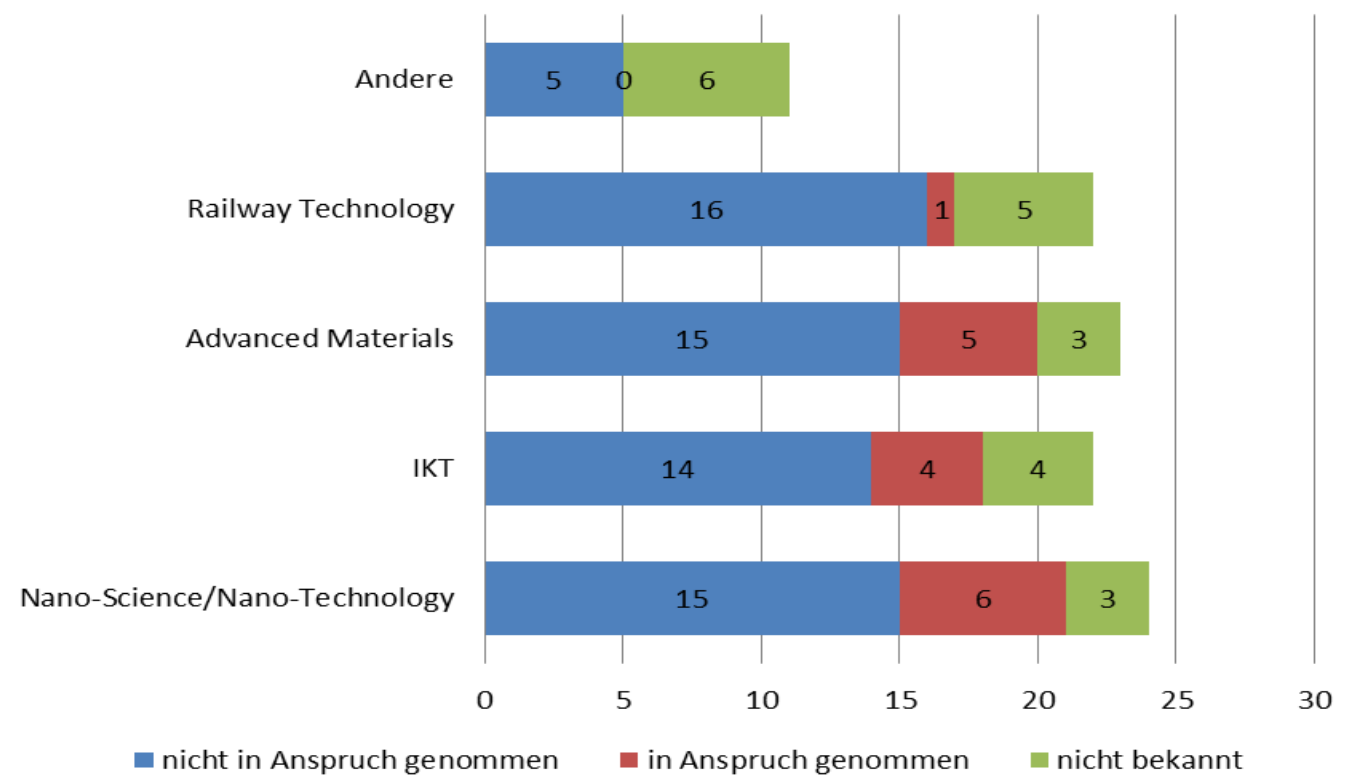

Quelle: JOANNEUM RESEARCH 


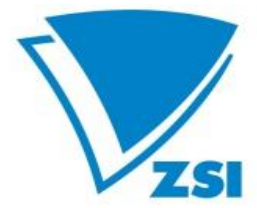

ZENTRUM FÜR SOZIALE INNOVATION

CENTRE FOR SOCIAL INNOVATION

Abbildung 8: Delegationsreisen OSTA Peking - Zufriedenheit

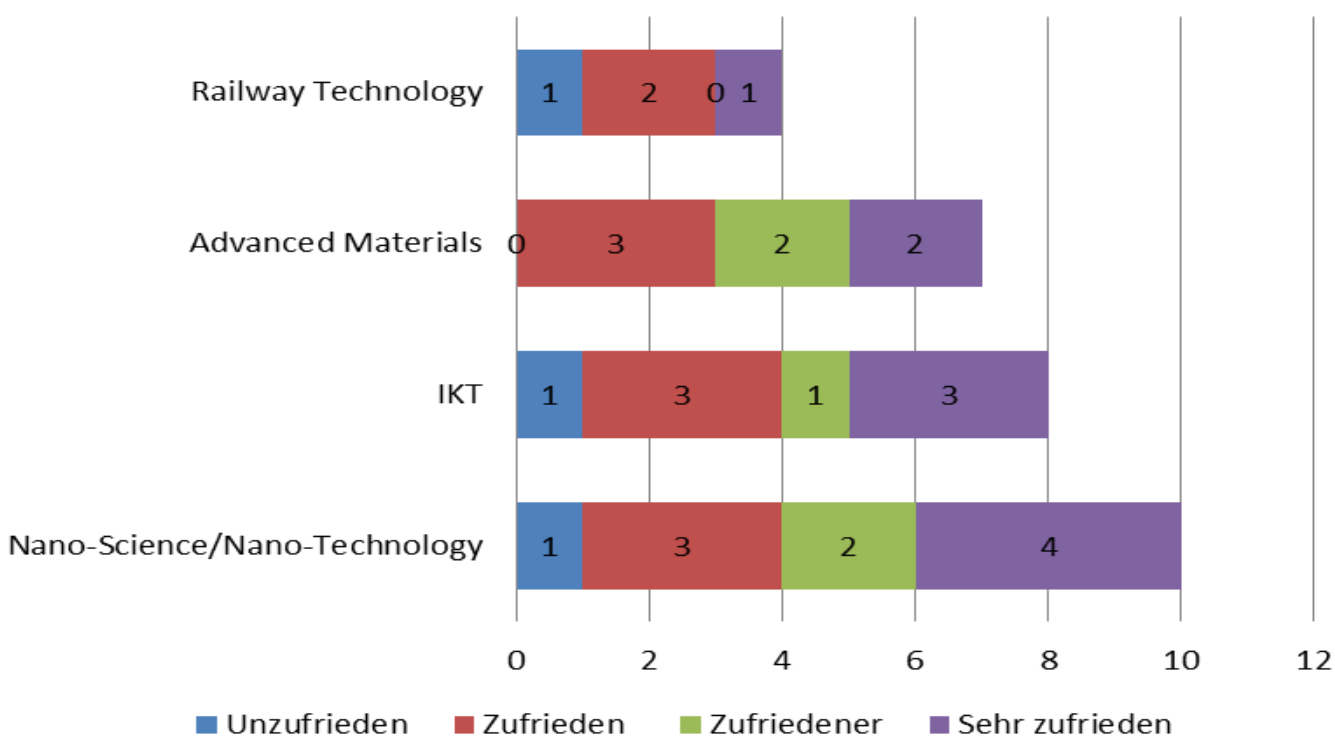

Quelle: JOANNEUM RESEARCH

Die Abbildungen zeigen die Inanspruchnahme sowie die Bewertung der Delegationsreisen vonseiten der Teilnehmenden des Online Surveys:

- Die zentralen Themen sind „Railway“, „Nano“, „Material“

- Die Antworten kommen aus der außeruniversitären Forschung, (4), aus dem BMVIT (2) und jeweils ein Unternehmen, eine Hochschule und eine Fördereinrichtung

- Die Zufriedenheit ist mehrheitlich positiv bis hoch

- Es gibt eine negative Rückmeldung aus dem Hochschulbereich

\subsection{Stakeholder}

Aus Perspektive der im online-Survey befragten Personen sind die wichtigsten Leistungsbereiche des OSTA Peking:

- Identifikation potenzieller Partner und Kontaktherstellung

- $\quad$ Unterstützung bzw. (Mit-)Organisation von Delegationen

- $\quad$ Anbahnung bilateraler Kooperation (Forschung/Unternehmen) 


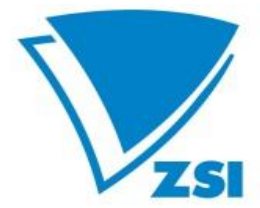

ZENTRUM FÜR SOZIALE INNOVATION

CENTRE FOR SOCIAL INNOVATION

Ebenfalls wichtig wird die Unterstützung internationaler Mobilität von Forschenden, die Unterstützung (FTI-)politischer bilateraler Kooperationen und die Daten- und Informationsbereitstellung zu China angesehen.

Wie auch bei OSTA Washington ergibt die Frage, welche Aufgaben künftig stärker wahrgenommen werden sollen, ein ähnliches Bild $(n=26)$.

Abbildung 9: Künftige Aufgaben OSTA Peking

Informationsbereitstellung, Analysen und Daten zu China

Identifikation von potentiellen Partnern und Herstellen von Kontakten

Anbahnung bilateraler Kooperation zwischen Forschungseinrichtungen und Unternehmen Unterstützung internationaler Mobilität (Austausch) von Forscherlnnen

Unterstützung bi- und multilateraler Kooperationen (z.B. in EU-Programmen)

Unterstützung des Ausbaus bilateraler Kooperationen auf (FTI-)politischer Ebene

Informationsbereitstellung, Analysen und Daten zum FTI-Standort Österreich

Vermarktung österreichischer Technologien in China

Betreuung und Beratung österreichischer Forschender in China

Unterstützung bzw. (Mit-)Organisation von Delegationen

Maßnahmen zur (FTI-)Politikunterstützung

Vermittlung von Jobangeboten in China

Betreuung und Beratung österreichischer Unternehmen in China

Betreuung und Beratung chinesischer Forschender in Österreich

Vermittlung von Jobangeboten in Österreich

Andere Aktivität

Betreuung und Beratung chinesischer Unternehmen in Österreich

In welchen der folgenden Bereiche sollte das OSTA Peking in Zukunft seine Aktivitäten verstärken?

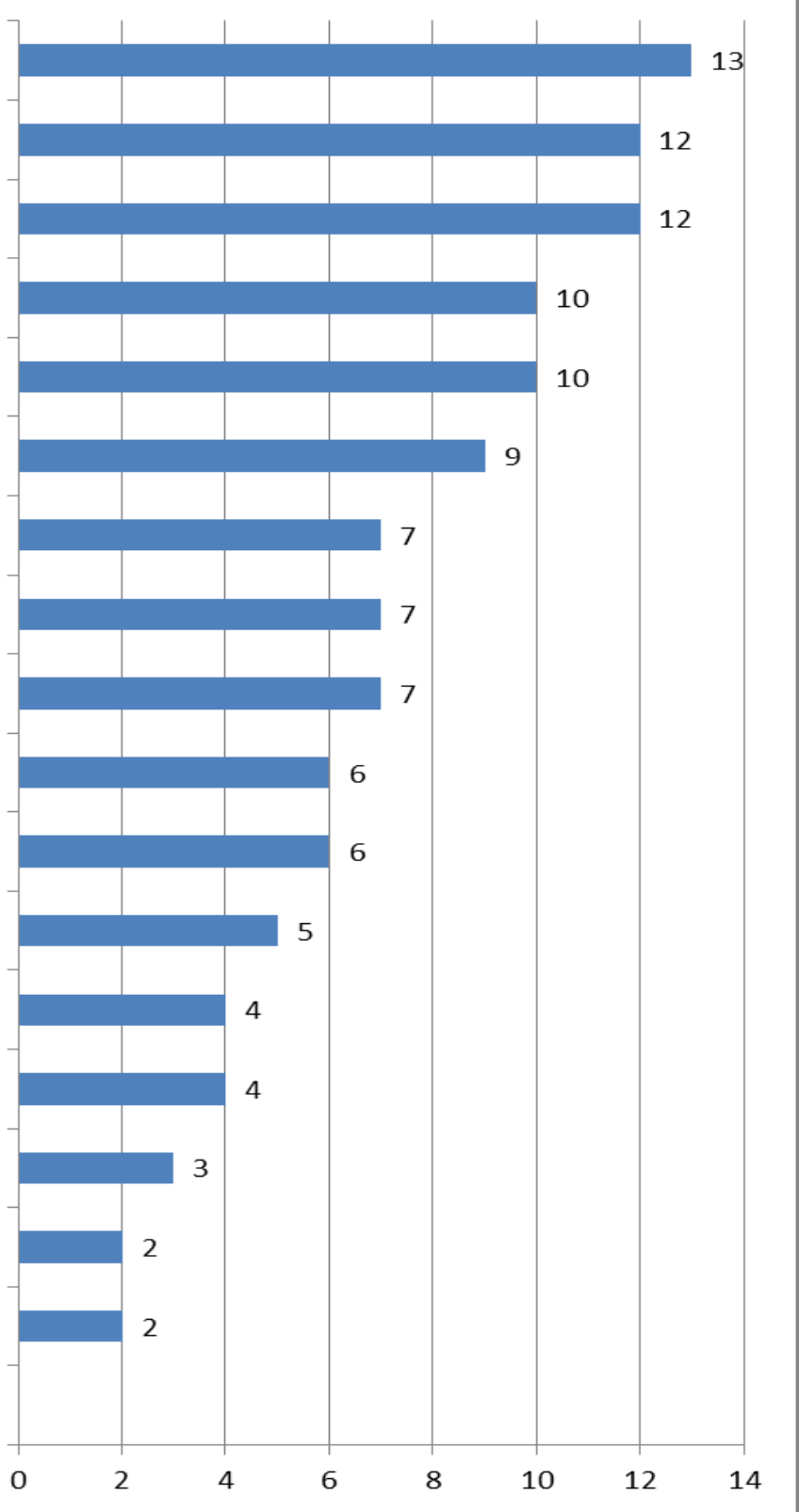

Quelle: JOANNEUM RESEARCH 


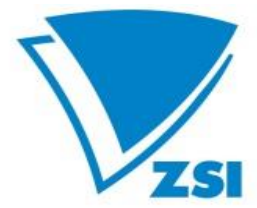

ZENTRUM FÜR SOZIALE INNOVATION

CENTRE FOR SOCIAL INNOVATION

Die Zufriedenheit mit den Aktivitäten des OSTA Peking ist ebenso wie bei OSTA Washington mehrheitlich hoch. Kritisch angemerkt wurde die mangelnde Information über und die mangelnde Anbindung an die Grundlagenforschung. Dem massiven Aufholen Chinas im Wissenschaftsbereich müsse stärker Rechnung getragen werden, äußerten mehrere Teilnehmende der Fokusgruppe: „Es wird immer noch so getan, als sei China ein Entwicklungsland".

Insgesamt äußerten sich sowohl einige interviewte Personen aus den Ressorts als auch die Teilnehmenden der Fokusgruppen für eine stärkere Konzentration auf FTI-Kooperationen und eine entsprechende Verschränkung mit kooperativen Forschungsprogrammen (COMET, CD Labors, Beyond Europe, thematische Programme des BMVIT).

Auch zeigte sich sowohl in der Fokusgruppe als auch bei einzelnen Interviews eine gewisse Diskrepanz in der Frage zum zukünftigen Fokus des OSTA Peking. In diesem Kontext wurden die folgenden Fragen diskutiert:

- Sollen die derzeit dominanten Aktivitäten im Bereich des Technologietransfers beibehalten und eventuell sogar gestärkt werden?

- Sollen Aktivitäten im Bereich des Forschenden-Austauschs und der Alumni-Pflege stärker ausgebaut werden?

- Wie kann eine stärkere Orientierung in Richtung (Grundlagen)forschung aussehen, vor allem angesichts dessen, dass Universitäten und Forschungseinrichtungen nur sehr wenig Interesse an der Arbeit von OSTA Peking zeigen?

- Können und sollen die Bemühungen verstärkt werden, die Mongolei zu adressieren? Derzeit scheint nach Einschätzung der meisten Stakeholder die Mongolei kaum anschlussfähig zu sein.

In den Interviews mit den Ressorts wurde mehrheitlich Zufriedenheit mit der Arbeit von OSTA Peking geäußert. Besonders positiv wurden die Serviceorientierung und das Engagement der Mitarbeitenden hervorgehoben. Kritische Aussagen gab es zu den folgenden Punkten:

- Der deutliche Fokus auf die Organisation von Delegationsreisen lässt für andere Aktivitäten wenig Raum. Einer der interviewten Personen meinte: „Ich brauche kein Reisebüro“.

- Ein konkreter Output wird vermisst: „Fünf österreichische Unternehmen, die mit Forschungseinrichtungen vor Ort zusammenarbeiten. Oder ein neuer Trend, eine neue Technologie, die für Österreich einen interessanten Input darstellen" 


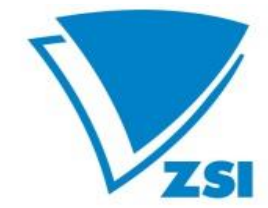

ZENTRUM FÜR SOZIALE INNOVATION

CENTRE FOR SOCIAL INNOVATION

- Bei einer gleichen finanziellen Belastung der beteiligten Ressorts stellt sich die Frage, ob auch alle in gleicher Weise von den Aktivitäten profitieren: "Wahrscheinlich wurde anderen Ministerien besser zugearbeitet".

\subsection{Alumni Datenbank}

Der Aufbau der Alumni-Datenbank hat sich verzögert: Zunächst dauerte die Ausarbeitung des Konzepts vonseiten OSTA Peking deutlich länger als geplant, dann mussten bei der Übermittlung einer DSGVO-kompatiblen Datenstrukturierung durch den OeAD an das OSTA einige technische Hürden überwunden werden. In der Alumni-Datenbank, die nun im OSTA liegt, sind 140 Alumni erfasst.

Es handelt sich bei den Alumni fast ausschließlich um chinesische Personen, die in Österreich studiert oder Kurzforschungsaufenthalte durchgeführt hatten. Damit die Datenbank sukzessive befüllt werden kann, werden alle Visumsantragstellenden aus dem FTI-Bereich in Zukunft gefragt, ob er oder sie sich in die Alumni Datenbank eintragen möchte (opt-in). Die Frage nach Eintragung in die Alumni Datenbank im Zuge der Visumserteilung wird als effizientes Hilfskonstrukt wahrgenommen. Idealerweise sollten die Universitäten selbst die Information über chinesische Studierende/Gastforschende bereitstellen, aber da scheint es laut OSTA keinen funktionierenden Mechanismus in Österreich zu geben. Das OSTA wünscht sich vom BMBWF oder vom OeAD in dieser Hinsicht intensivere Ansprache der österreichischen Universitäten. Manche Universitäten, wie die TUWien haben sogar schon ihre eigenen Alumni-Netzwerke (z.B. in Shanghai).

Die Zusammenarbeit mit dem OeAD-Büro in Shanghai in Bezug auf die Alumni Datenbank (und darüber auch hinaus) scheint sehr gut zu funktionieren und von gegenseitigem Respekt getragen zu sein.

Die Alumni-Datenbank wird in erster Linie für die Alumni-Tage verwendet. OSTA denkt daran, nicht nur in Peking und Shanghai Alumni-Tage abzuhalten, aber es sind sowohl die eigenen Ressourcen als auch die kritischen Massen vor Ort in der Provinz knapp, weshalb man einer Ausweitung der AlumniTage unentschieden gegenübersteht (im Gegensatz zum OeAD-Büro in Shanghai, dass hier explizit eine Ausweitung wünscht und auch in der Zusammenarbeit mit Deutschland Synergien sieht).

Die Alumni sind auch auf einer WeChat-Gruppe vereint, da die Selbstvernetzung als wichtig anerkannt wird. Sie erfahren Neuigkeiten über Österreich, sowie über Feiertage und Messen. Es wird nichts Privates kommuniziert und auch nichts Politisches. Sponsoren dürfen Informationen posten, aber 


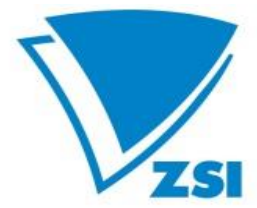

ZENTRUM FÜR SOZIALE INNOVATION

CENTRE FOR SOCIAL INNOVATION

keine direkte Werbung. OeAD hat die Editioralrechte für die WeChat-Gruppe und kontrolliert daher jedes Posting bevor es abgesetzt wird.

Eine Dokumentation von Alumni Zahlen zu einem fixen Stichtag sollte angeregt werden. Ebenso ist eine Dokumentation der Anzahl der Aussendungen zu überlegen. Bezüglich Kontaktvermittlung ist eine Dokumentation schwierig bis unmöglich, weil die Alumni über die WeChat-Gruppe vernetzt sind und damit die Möglichkeit haben, selbst untereinander in Austausch zu treten. Jobpostings gibt es keine und damit werden auch keine Jobs über die WeChat-Gruppe vermittelt. Advantage Austria hat auch eine WeChat-Gruppe und dort werden im Unterschied zum OSTA Jobpostings veröffentlicht.

\subsection{Governance vor Ort, Abstimmung mit anderen Akteuren}

\section{Governance:}

Ähnlich wie beim OSTA Washington wird auch beim OSTA Peking die Eingliederung in die Botschaft fast einhellig als sinnvoll angesehen. Der Bedarf zusätzlicher Regelungen von Verantwortungsbereichen mittels einer eigenen Geschäftsordnung wird im Unterschied zu OSTA Washington nicht vorrangig angesehen. Das Handbuch des Auswärtigen Dienstes dient hier quasi als Geschäftsordnung und wird als ausreichende Arbeitsgrundlage wahrgenommen.

Das Problem der Leitung vonseiten einer Person aus dem höheren auswärtigen Dienst existiert in Peking in ganz gleicher Weise wie in Washington. Auch hier gab es bei der letzten Ausschreibung für OSTA Peking nur eine Bewerbung.

\section{Abstimmung vor Ort:}

Die Kooperation mit AußenwirtschaftsCentern (AWC) wird sowohl in China als auch in Österreich als problematisch angesehen: Im Interview vor Ort wird die bessere nachhaltige Betreuung und Anschlussfähigkeit durch das AWC betont und ein sensibleres Erwartungsmanagement vonseiten des OSTA gewünscht. B2b wird als rote Linie gesehen, bei $b 2 p$ agieren OSTA und AWC häufig wenig abgestimmt. Auch in Österreich äußern sich sowohl Ressortvertretungen als auch Vertreter der WKÖ kritisch zur Konkurrenz zwischen OSTA und den AWC:

- „OSTA Peking setzt Aktivitäten, ohne über ausreichend Netzwerke zu verfügen. Das sind Grenzüberschreitungen. Man kann und soll z.B. nicht zwischen Technologieexport, normalen Export und FDI trennen." 


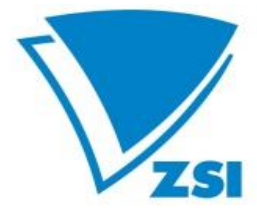

ZENTRUM FÜR SOZIALE INNOVATION

CENTRE FOR SOCIAL INNOVATION

- „Wir wollen, dass die gescheit zusammenarbeiten und sich nicht gegenseitig konkurrenzieren"

Die Kooperation mit dem OeAD-Office in Shanghai läuft sehr gut, vor allem auch, weil das OeAD ein klares Profil in Richtung Hochschulmobilität und GSK sowie einen klaren regionalen Fokus aufweist. Mögliche Konfliktpunkte sind aufgrund der Komplementarität der beiden Einrichtungen nur wenig gegeben. Die Zusammenarbeit bei der Alumni-Betreuung, beim "nation branding“ und bei der regionalen Öffentlichkeitsarbeit funktioniert im gegenseitigen Einverständnis und zum beiderseitigen Nutzen.

Die Kooperation mit EU-Delegationen im FTI-Bereich ist eine für die österreichische Botschaft wichtige Funktion des OSTA und wurde in den letzten Jahren auch deutlich professionalisiert.

Die Kooperation mit EUPIC (EU Project Innovation Centre) war gut. OSTA organisierte im Jahr 2018 die Unterzeichnung des MoU zwischen FFG und EUPIC durch BM Schramböck im Rahmen eines Staatsbesuchs einer rund 250-köpfigen österreichischen Delegation in China, bei dem der Ausbau bilateraler Beziehungen in Wissenschaft und Wirtschaft diskutiert wurde. Ein Personalwechsel bei EUPIC hat das Kooperationsniveau jedoch reduziert.

Einschätzung: Insgesamt funktioniert die Zusammenarbeit mit dem OeAD-Büro in Shanghai gut, in der Kooperation mit den AWC gibt es Luft nach oben. Da das OSTA Peking seine Kernklientel ebenso in Unternehmen mit explizitem Technologiebezug sieht, gibt es diesbezüglich eine kaum auflösbare Überschneidung mit AWC. OSTA Peking könnte sich stärker auf wissenschaftlich-institutionelle Kooperation konzentrieren, wie dies auch von der Mehrzahl der Stakeholder bei der Fokusgruppe vorgeschlagen wurde. Allerdings ist die Bekanntheit und Anschlussfähigkeit des OSTA Peking in Österreich zurzeit nur bedingt gegeben. Positiv ist zu vermerken, dass das OSTA Peking vom offiziellen China vor Ort als erster Ansprechpartner für wissenschaftlich-technische Kooperation wahrgenommen wird.

\subsection{Verwaltung und Gebarung}

\section{Büroorganisation:}




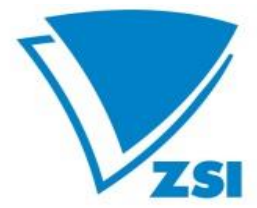

ZENTRUM FÜR SOZIALE INNOVATION

CENTRE FOR SOCIAL INNOVATION

Insgesamt funktioniert das Bürogefüge in Peking sehr gut. Es gibt im Unterschied zu Washington keine Zuordnung der Mitarbeitenden zu Themen und Ressorts, alle Aktivitäten sind bei Leitung und Stellvertretung gebündelt. Das chinesische Personal wird nur unterstützend eingesetzt (Administration und Unterstützung bei der Organisation von Dienstreisen sowie Kontaktaufnahmen und -durchführungen). Hier könnten vereinzelt auch selbstständigere und anspruchsvollere Tätigkeiten übernommen werden.

Die Mitarbeitenden sind hoch motiviert und auch angemessen qualifiziert, die Arbeitsbelastung ist allerdings groß. 20\% der Dienstzeit von Herrn Dr. Agothonos wird für Reisetätigkeiten aufgewendet, für Herrn Pfeiler ist das deutlich weniger. Beide haben viele Überstunden, die abgebaut werden müssen.

Während die Vernetzung in China recht gut und breit ist, fehlt es an Bekanntheit und Anschlussfähigkeit in Österreich - eine Ausnahme stellt hier das BMVIT dar.

\section{Gebarung}

Die Buchhaltung sowie die Aufbereitung und Archivierung der Abrechnungen erfolgen ordnungsgemäß. Die vielen Dienstreisen werden effizient organisiert: Preise werden online verglichen, zugekaufte Services sind marktkonform (wenngleich nicht immer 3 Angebote eingeholt werden).

Die geprüften Bereiche waren fehlerfrei:

- Salden und Konsistenz der Einnahmen-Ausgaben Rechnung

- Handkassa existiert und wurde geprüft

\section{Governance in Österreich}

Die interministerielle Governance der vier Ministerien BMEIA, BMVIT, BMBWF und BMDW wird seitens der Ressorts als komplex und zeitaufwändig wahrgenommen. Eine der interviewten Personen merkt an: „Die Konstruktion ist prinzipiell sinnvoll, der Abstimmungsaufwand allerdings enorm. “Eine andere: „OSTA Washington macht uns mehr Arbeit als es uns abnimmt". Von Seiten der Stakeholder wurde befunden: „Prinzipiell ist die gemeinsame Verantwortung aller involvierten Ressorts positiv.“ 


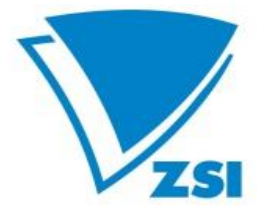

ZENTRUM FÜR SOZIALE INNOVATION

CENTRE FOR SOCIAL INNOVATION

Der Lenkungsausschuss trifft sich in der Regel zweimal jährlich. Einmal im Jahr berichten die Direktoren von OSTA Washington und Peking über ihre Aktivitäten und stellen die Pläne für das kommende Jahr vor. Dabei geht es teilweise um größere Weichenstellungen und Orientierungen, teilweise aber auch um sehr kleinteilige Vorhaben wie beispielsweise die Teilnahme an einzelnen Veranstaltungen oder auch um Probleme mit der Einbindung in die IT-Systeme der Botschaften.

Durch die sehr klare und ähnliche Struktur der Arbeitsprogramme und Jahresberichte sind die Leistungen der OSTA für die verantwortlichen Ressorts deutlich erkennbar. Korrekturen und künftige Wünsche können formuliert und in der Folge umgesetzt werden. Allerdings ist der Spielraum für zusätzliche Aktivitäten recht begrenzt, da die zur Verfügung stehenden Budgets lediglich die laufenden Ausgaben decken. Zusätzliche Wünsche, Vorschläge und Projekte können meist nicht finanziert werden. Auch wenn die Umsetzung der Arbeitsprogramme vonseiten der Ressorts überwiegend zufriedenstellend bewertet wird, gibt es vereinzelt kritische Stimmen zur Serviceorientierung der OSTA: „Der Besuch der Ministerin war wenig gut servisiert”.

Die OSTA wiederum vermissen einerseits konkrete Wünsche und Schwerpunktsetzungen der Ressorts, andererseits fällt ihnen die Übernahme neuer Orientierungen schwer. Beispielsweise vermisst das BMVIT eine dezidierte Orientierung auf Technologietransfer vonseiten OSTA Washington; OSTA Peking sieht eigene Defizite im Wissenschaftsbereich (dies wurde vonseiten zentraler Stakeholder in der Fokusgruppe bestätigt).

Die Abrechnungskontrolle seitens BMBWF (Stefanie Nagel) funktioniert gut, ist allerdings auch aufwändig: „und eigentlich ist es nicht ihr Job.“

\section{Anregungen:}

Um der als aufwändig wahrgenommenen Steuerung entgegen zu wirken, könnte sich der Lenkungsausschuss künftig auf größere und längerfristige Fragestellungen bzw. Geschäftsfelder konzentrieren. Dazu gehört auch die Umstellung auf mehrjährige Planungshorizonte (siehe Abschnitt 5.2) und die Auslagerung der operativen Kontrolle beispielsweise an eine Agentur oder Trägereinrichtung. Auch könnte überlegt werden, die Abrechnungen durch die Kanzleien der Botschaften durchführen zu lassen - in Peking fehlen dazu allerdings derzeit die Ressourcen. 


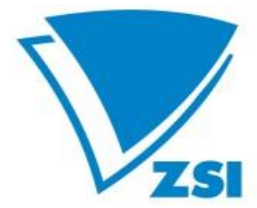

\section{Schlussfolgerungen und Empfehlungen}

Aus der genauen Analyse der OSTA, den internationalen Beispielen, den Interviews mit den Ressorts, den Fokusgruppen mit Stakeholdern sowie dem Online Survey lassen sich die folgenden Empfehlungen für die Zukunft der OSTA ableiten:

\section{Klare Positionierung, Definition der Ziele, der Agenda und der strategischen Orientierung:}

Unabhängig von den vielen und vielfältigen Einzelaktivitäten, die derzeit von beiden OSTA durchaus engagiert durchgeführt werden, sollten sich die Ressorts sehr grundsätzlich überlegen, was sie von den OSTA erwarten und wünschen. Dazu gehört auch eine stärkere Orientierung an existierenden Strategien wie beispielsweise der Vorschlag zur „Beyond Europe“ Strategie oder die Außenhandelsstrategie sowie an der in Arbeit befindlichen FTI Strategie. OSTA Peking wurde als gemeinsame Aktivität der Ressorts in Umsetzung der aktuellen FTI-Strategie eingerichtet, dennoch lassen Programme und Aktivitäten beider OSTA eine explizite Ausrichtung an der Strategie bislang vermissen.

Die Positionierung beider OSTA sollte im wissenschaftlich-technischen Bereich liegen, und vor allem FTI-Kooperationen unterstützen, und zwar zwischen Forschenden und wissenschaftlichen Einrichtungen in Österreich und den Sitzländern als auch zwischen technologieaffinen Unternehmen und wissenschaftlichen Einrichtungen. Mit dieser Orientierung hätten beide OSTA klare Alleinstellungsmerkmale und wären attraktive Kooperationspartner sowohl für die AWC in beiden Regionen als auch für die OeAD in China und ASciNA in Nordamerika. Dabei können und sollen sich die beiden Profile aufgrund der unterschiedlichen Situation - vor allem der Existenz einer wissenschaftlichen Diaspora in Nordamerika - durchaus unterscheiden. Wichtig ist eine grundsätzliche und auch längerfristige Orientierung.

\section{Anschlussfähigkeit in Österreich verbessern}

Derzeit ist die Arbeit und Zielsetzung beider OSTA in Österreich wenig sichtbar und bekannt. Dies gilt vor allem für die Hochschulen, die in der Regel über eigene Netzwerke und Kontakte in den nordamerikanischen und chinesischen Raum verfügen. Das gilt aber auch - von wenigen Ausnahmen abgesehen - für große Unternehmen und Forschungseinrichtungen. Hier könnte eine bessere Einbindung der Stakeholder über einen Beirat, beispielsweise ähnlich dem swissnex Beirat, die Verankerung in Österreich verbessern. Auch eine intensivere Nutzung der temporären Entsendungsmöglichkeit oder periodische Treffen in Wien und Alpbach könnten die Sichtbarkeit in 


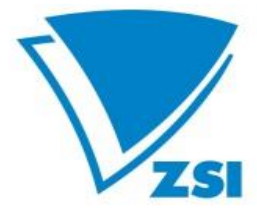

ZENTRUM FÜR SOZIALE INNOVATION

CENTRE FOR SOCIAL INNOVATION

Österreich erhöhen. Die OSTA sollten sich deutlich systematischer potenziellen Kundinnen und Kundinnen sowie Partnern und Partnerinnen vorstellen. Dazu geeignet wären eine Roadshow bzw. breiter kommunizierte Kontakttage in Österreich. Auch empfiehlt sich eine strukturelle Kooperation mit den einschlägigen österreichischen Agenturen zur Verbreitung und für die Mobilisierung österreichischer Stakeholder bei gewissen Anlässen.

\section{Kooperation mit und Abgrenzung zu den AußenwirtschaftsCentern (AWC) definieren}

Während in Washington das OSTA mit den AWC aufgrund der komplementären Aufgabengebiete durchaus gut zusammenarbeitet, ist das Verhältnis in Peking eher von Konkurrenz geprägt. Derzeit sind Aktivitäten im Bereich b2b den AWC vorbehalten. Eine bessere Kooperation könnte durch die Konzentration der OSTA auf Kooperationsprojekte an der Schnittstelle von Wissenschaft und Wirtschaft auch in Verbindung mit existierenden FTI Programmen gelingen. Auch im Bereich öffentlicher Infrastrukturen (Verkehr, Smart Cities etc.), in dem OSTA Peking bereits heute durchaus aktiv ist, gäbe es unter stärkerer Einbindung der Universitäten und Forschungseinrichtungen beider Seiten noch weitere Betätigungsfelder. Auch eine räumlich engere Zusammenarbeit inklusive personeller Verschränkungen (wie beispielsweise Team Sweden) könnten angedacht werden.

\section{Berichtswesen und Reporting optimieren}

Planung und Reporting sind durch die einheitliche und klare Struktur von Arbeitsprogrammen und Jahresberichten bereits heute gut geregelt. Weitere Verbesserungen könnten durch eine Umstellung der Planung in strategisch orientiert Mehrjahresprogramme in Kombination mit kurzen Jahresprogrammen und -berichten erreicht werden. Einheitliche Templates würden die Übersichtlichkeit zusätzlich erhöhen.

\section{Reduktion des operativen Aufwandes auf allen Seiten}

Der operative Aufwand ist für alle Beteiligten recht hoch und alle künftigen Lösungen für die OSTA sollten Maßnahmen beinhalten, diesen zu reduzieren. Eine Möglichkeit wäre beispielsweise die Auslagerung der operativen Tätigkeiten an eine professionelle Agentur oder Trägereinrichtung in Österreich.

Im Folgenden werden vier Szenarien für eine künftige Organisation der OSTA vorgestellt: 


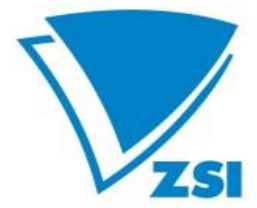

ZENTRUM FÜR SOZIALE INNOVATION

CENTRE FOR SOCIAL ININOVATION

\subsection{Szenario 1: kleine OSTA mit variabler Geometrie}

Dieses Szenario kommt der derzeitigen Organisation der OSTA am nächsten: Nach wie vor ist OSTA Teil der Botschaft und wird von einer aus dem BMEIA entsendeten Person geleitet, deren primäre Aufgabe darin besteht, die diplomatischen Beziehungen zwischen dem Sitzland und Österreich im FTI Bereich zu stärken (Science Diplomacy bzw. STI Diplomacy). Die Fachressorts BMBWF, BMDW und BMVIT können zusätzliches Personal entsenden oder finanzieren (inklusive einem Overhead für die Infrastrukturkosten), das ihre jeweiligen Interessen vertritt. OSTA kooperiert vor Ort mit den AWC und anderen lokalen Partnern, in Österreich mit verschiedenen Stakeholdern aus dem FTI Bereich wie Hochschulen, Unternehmen, Forschungseinrichtungen und Agenturen.

\section{Szenario 1: kleine OSTA mit variabler Geometrie}

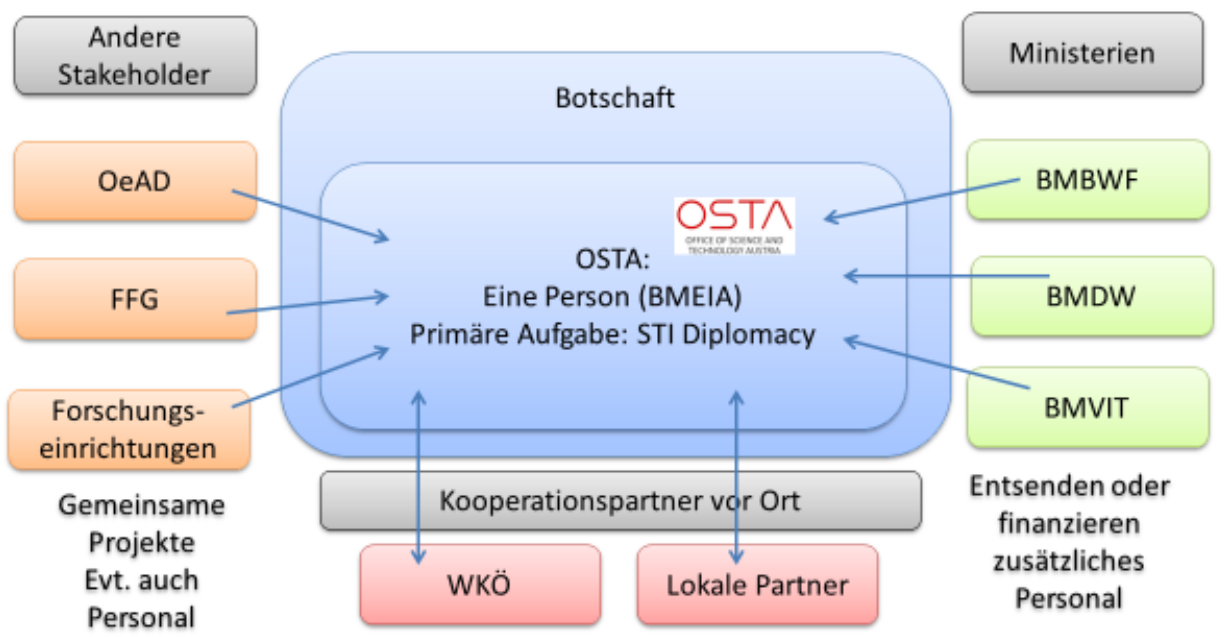

Quelle: Eigene Darstellung

Die Vorteile dieses Szenarios sind:

- Höhere Flexibilität: Thematisch, zeitlich und hinsichtlich der adressierten Zielländer

- Geringere Anforderungen an die Co-Governance

- Klare Zuständigkeiten und Verantwortungsbereiche der Ministerien

- Größere Freiheitsgrade bei der Auswahl der Personen 


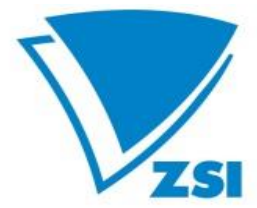

ZENTRUM FÜR SOZIALE INNOVATION

CENTRE FOR SOCIAL ININOVATION

Die Nachteile hingegen:

- Kein stabiles Team mit erfahrenen Personen

- Größere gemeinsame Aufgaben und Infrastrukturen (Datenbanken) sind schwieriger zu bewältigen

- Geringe Kontinuität und Stabilität

- Geringe Sichtbarkeit

\subsection{Szenario 2: Bürogemeinschaft von Attachés}

In Szenario 2 entfällt die Leitung durch eine vom BMEIA entsendeten Person. Stattdessen entsenden oder nominieren alle Ressorts Attachés bzw. Attachées für Wissenschaft, Technologie und Innovation, die in der Botschaft verortet sind. Die Infrastrukturkosten werden gemeinschaftlich getragen. Es empfiehlt sich, dass sich die Ressorts in Bezug auf die entsendeten Personen, deren Aufgabengebiete und die Dauer ihres Einsatzes abstimmen. Wie in Szenario 1 kooperiert diese Gemeinschaft von Attachés mit Partnern vor Ort und Stakeholdern in Österreich. Die Wissenschafts- und Technologie Attachés müssten zudem gut vernetzt sein (zueinander und in Richtung Österreich). Hier käme den FTI-Ministerien eine wichtige Aufgabe zu woraus sich wiederum Anforderungen an eine gewisse CoGovernance ergäben.

Abbildung 11: Szenario 2 - Bürogemeinschaft von Attachés

\section{Szenario 2: Bürogemeinschaft von Attachés}

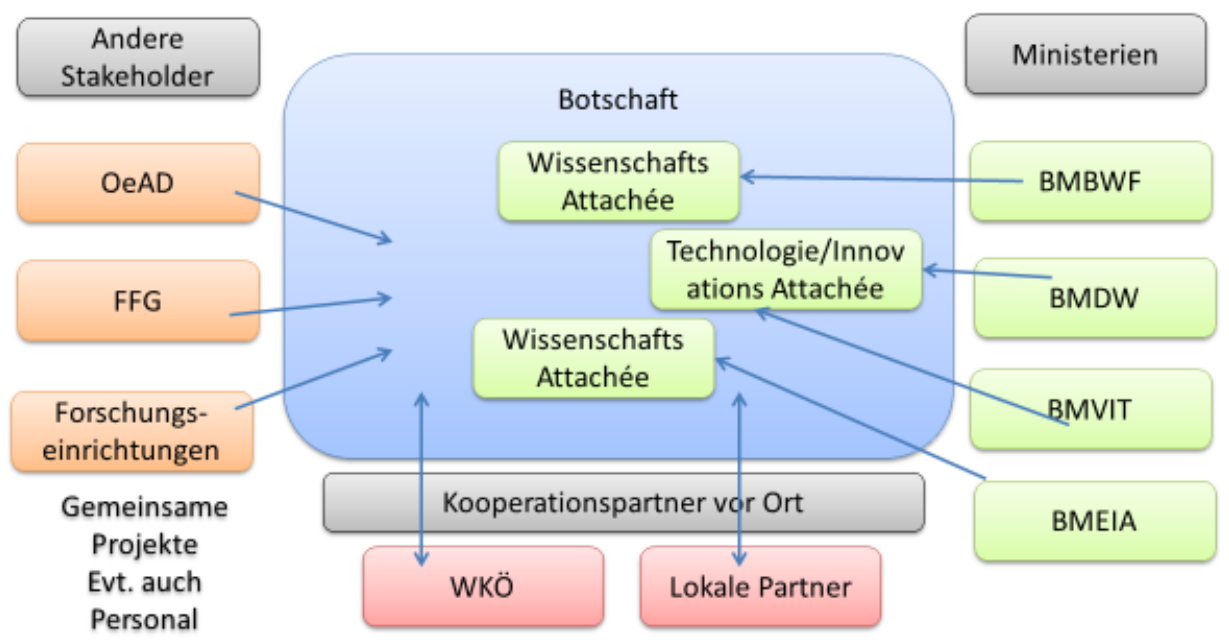

Quelle: Eigene Darstellung 


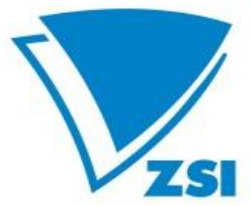

ZENTRUM FÜR SOZIALE INNOVATION

CENTRE FOR SOCIAL ININOVATION

Die Vorteile von Szenario 2 sind:

- Sehr hohe Flexibilität: Thematisch, zeitlich und hinsichtlich der adressierten Zielländer

- Keine bzw. geringe Anforderungen an die Co-Governance

- Klare Zuständigkeiten und Verantwortungsbereiche der Ministerien

Die Nachteile hingegen:

- Teuer (Attachés kosten wesentlich mehr als Angestellte eines OSTA)

- Kein stabiles Team mit erfahrenen Personen

- Keine Leitung und interne Governance

- Geringe Kontinuität, Stabilität und Sichtbarkeit

\subsection{Szenario 3: Trägerlösung}

Alternativ dazu kann eine Agentur bzw. eine Trägereinrichtung beauftragt werden, ein OSTA einzurichten oder zu betreiben. Dazu definieren die Ressorts ToRs, welche Aufgaben das OSTA zu erfüllen hat, welche Kompetenzen vorhanden sein müssen und wie Governance, Reporting, Budgetierung etc. zu erfolgen haben. Das OSTA kann, muss aber nicht in der Botschaft angesiedelt sein. Die räumliche - oder auch organisatorische - Anbindung an die Botschaft empfiehlt sich allerdings, um die Verbindung zur österreichischen Vertretung im Sitzland zu garantieren und somit die wichtige Funktion von "Science Diplomacy“ oder auch "STI Diplomacy“ erfüllen zu können. Szenario 3 kann auch als „Plattform“ oder „Team Österreich“ weitergedacht werden mit allen österreichischen Playern vor Ort unter einem Dach - analog zu „Team Sweden“ 


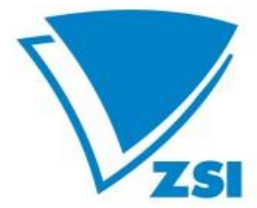

\section{Szenario 3: Trägerlösung}

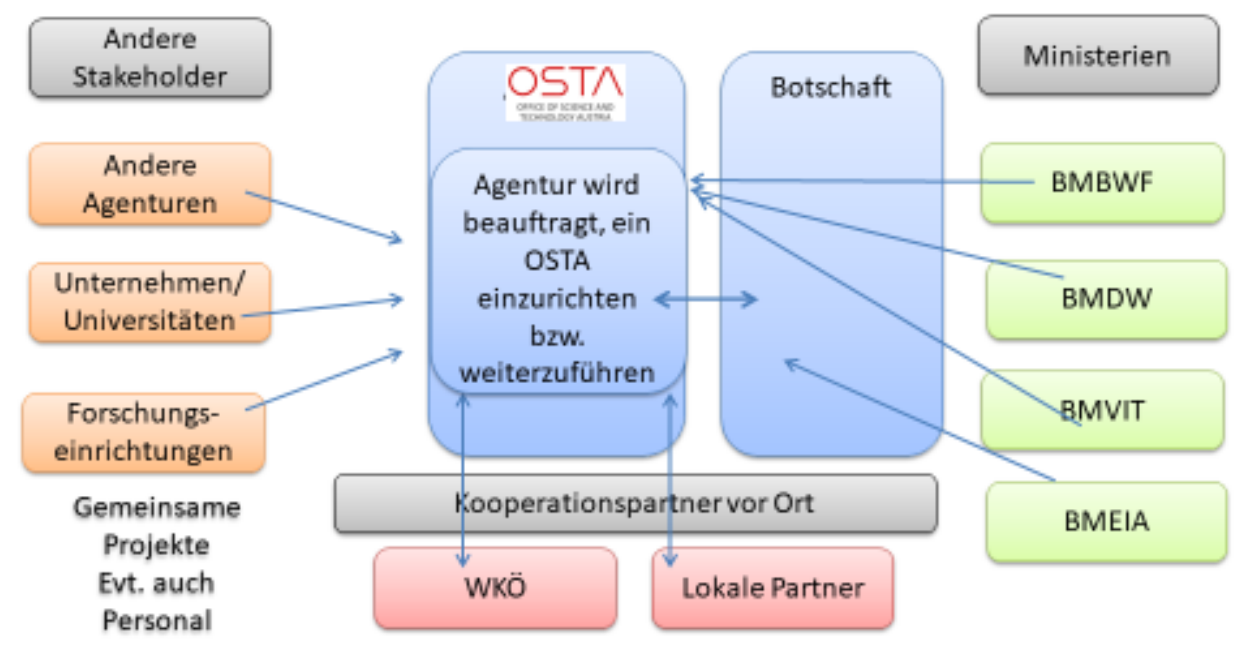

Quelle: Eigene Darstellung

Die Vorteile von Szenario 3 sind:

- Administrative Entlastung der Ministerien

- Größere Freiheitsgrade in der Auswahl der Personen

- Bessere Verbindungen zu nationalen und internationalen Förderformaten

Die Nachteile hingegen:

- Möglicherweise geringe Anbindung an das offizielle „Auslandsösterreich“, falls eine Lösung gewählt wird, die keine räumliche und/oder organisatorische Anbindung an die Botschaft beinhaltet

- Sichtbarkeit und diplomatische Anbindung fraglich

- Co-Governance der Ministerien auf Metaebene weiterhin notwendig

Im Falle von Szenario 3 sollte eine gemeinsame Budgetierung der beteiligten Ministerien angestrebt werden.

\subsection{Szenario 4: Internationalisierungsprogramm}

In diesem Szenario gibt es keine OSTA mehr. Stattdessen wird ein Internationalisierungsprogramm mit etwa fünf strategisch wichtigen Themenfeldern kompetitiv ausgeschrieben. Die Bewerber 


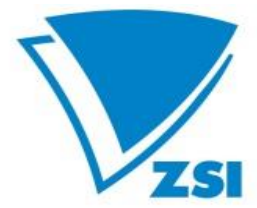

ZENTRUM FÜR SOZIALE INNOVATION

CENTRE FOR SOCIAL ININOVATION

müssen einen umfassenden Leistungskatalog entwickeln und eine kritische Masse an Stakeholdern in Österreich einbinden. In den Zielländern (bevorzugt aus Kreis 1 oder 2 der Empfehlungen zur Beyond Europe Strategie) werden Liaison-offices vor Ort eingerichtet - z.B. in Kooperation mit EUPIC oder Botschaft oder AWC und das vorab definierte Arbeitsprogramm umgesetzt. Nach einer etwa fünfjährigen Programmlaufzeit kann eine Wiederbewerbung erfolgen.

Abbildung 13: Szenario 4 - Internationalisierungsprogramm

\section{Szenario 4: Internationalisierungsprogramm}

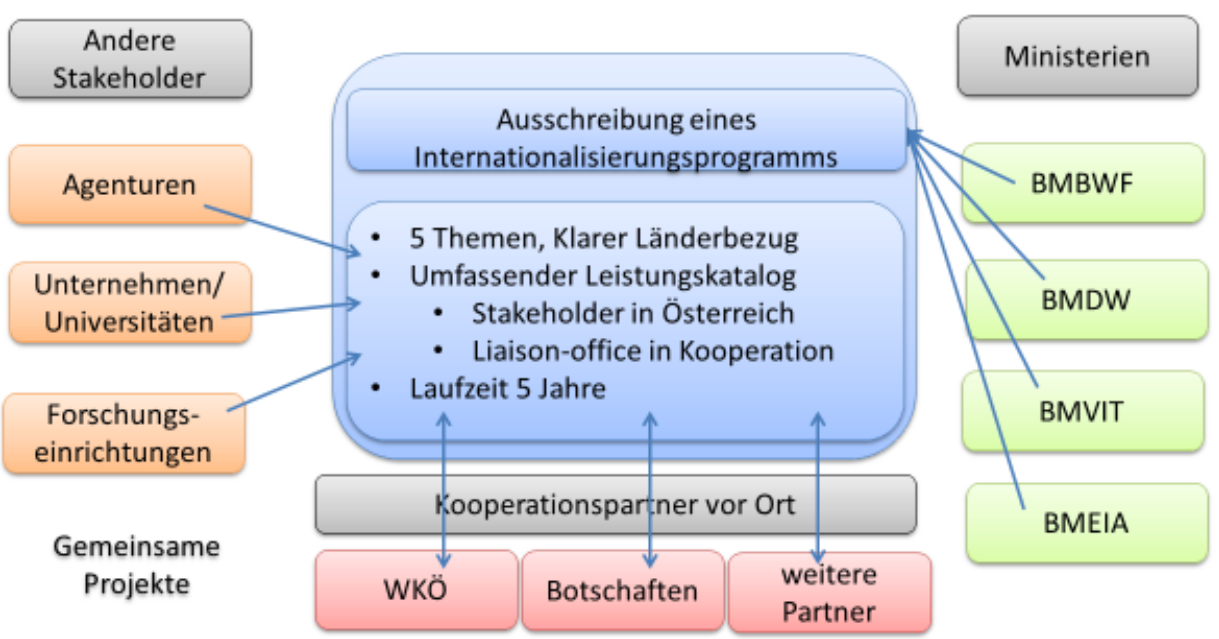

Quelle: Eigene Darstellung

Die Vorteile dieses Szenarios sind:

- Eine Orientierung am Bedarf und den Wünschen der FTI-Gemeinschaft ist als Voraussetzung gegeben

- Selbststeuerung durch die Partnerorganisationen mit klarem Arbeitsauftrag/ Programm und Wirkungsindikatorik (auf Basis kompetitiv eingereichter Vorschläge)

- Zeitlich limitiert und damit flexibel für zukünftige Entwicklungen (neue Ausschreibung nach vier Jahren)

- Kein unmittelbarer Co-Governance-Aufwand der Ministerien

Die Nachteile hingegen:

- Geringere Anbindung an Österreich (Botschaft; Ministerien)

- Evt. höhere Kosten 


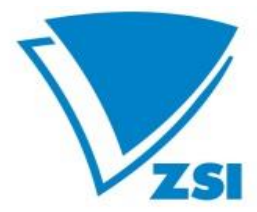

ZENTRUM FÜR SOZIALE INNOVATION

CENTRE FOR SOCIAL ININOVATION

- Längere Vorbereitungszeit

- Evt. keine ausreichend kompetitive Nachfrage

Auch im Falle von Szenario 4 sollte eine gemeinsame Budgetierung der beteiligten Ministerien angestrebt werden. Es könnten aber auch ressortspezifische Schwerpunkte und damit Finanzierungsbeteiligungen gesetzt werden.

\section{Die Beantwortung der Evaluierungsfragen}

A. Das OSTA-System im internationalen Vergleich

- Betreiben wesentliche FTI-Nationen wissenschaftliche Außenvertretungen?

- Vergleich der OSTA mit den Außenvertretungen anderer FTI-Nationen, bspw. Schweiz und Schweden

Nahezu alle europäischen Länder und auch einzelne Organisationen betreiben wissenschaftliche Außenvertretungen. Eine genaue Darstellung der Außenvertretungen Schwedens und der Schweiz findet sich in Kapitel 5.

\section{B. Wirkungsindikatoren der OSTA}

- Sichtbarkeit/Bekanntheit der OSTA sowohl in Österreich als auch im jeweiligen Sitzland

- Impact/Wirkung der Tätigkeiten der Büros im jeweiligen Sitzland

Beispielfragen:

- Wie wird der Impact gemessen?

- Wirkung/Nachhaltigkeit des Netzwerkes

Beispielfragen:

○ Wie nachhaltig ist der Kontakt zu jenen Personen, die nach Österreich zurückkehren?

\section{Wirkungsindikatoren:}

In Kapitel 4 sowie im Anhang finden sich Wirkungsindikatoren im Rahmen von Wirkungsmodellen entlang einer Output - Outcome - Impact Logik

\section{Sichtbarkeit:}

Die Sichtbarkeit beider OSTA in Österreich ist sehr gering. Es gibt kaum Nennungen auf den Webseiten der österreichischen Universitäten, Fachhochschulen und außeruniversitären Forschungseinrichtungen. Die mangelnde Anschlussfähigkeit an die österreichische FTI-Gemeinschaft ist eines der zentralen Ergebnisse aus den Fokusgruppen mit zentralen Stakeholdern. In den Sitzländern hingegen sind die OSTA sichtbar und weitgehend gut bekannt. 


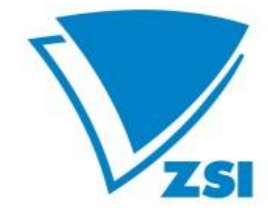

\section{Impact im Sitzland:}

Beide OSTA haben keine strukturierte Impact-Messung. Als Erfolg gilt, wenn die mit den Eigentümern vereinbarte Arbeit zur Zufriedenheit durchgeführt werden konnte. Ein systematisches Monitoring der follow-ups von Anbahnungen, die über die OSTA gelaufen sind (oder mit dessen Hilfe implementiert wurden) gibt es nicht, u.a. weil der Aufwand zum Teil unverhältnismäßig groß wäre (so möchten z.B. manche KundInnen keine Auskünfte erteilen-, secrecy') und daher keine lückenlose Abdeckung möglich erscheint. Man erfährt über follow-ups eher anekdotisch.

Laut OSTA Peking soll ein Wirkungsmonitoring v.a. auf neue Kanäle, neue Abkommen und Geschäftsfelder orientiert sein und die Wirkung derselben messen. Wirkung sollte auch messen, was Sinn gemacht hat und was nicht.

\section{Wirkung/Nachhaltigkeit:}

OSTA Washington: Die Kontaktpflege zu Personen, die nach Österreich zurückkehrten, wird sehr ernst genommen. In der Datenbank RINA gibt es eine eigene Kategorie für Alumni (siehe Abschnitt 7.5).

OSTA Peking: In Bezug auf Personen, die nach Österreich zurückkehren, z.B. von Delegationen oder aufgrund eines vorübergehenden expat-Statuses, wird - sofern es einen in China mit dem OSTA gab der Kontakt überwiegend weitergepflegt. Es gibt aber immer wieder Personen und Institutionen, die in China im einschlägigen Tätigkeitsfeld des OSTA Peking tätig sind und keinen Kontakt mit dem OSTA hatten.

C. Konsistenz von Arbeitsprogramm/Jahresbericht/Budget- und Personalverwendung und effizienter Einsatz der Mittel

- Umsetzung des Arbeitsprogrammes - Status der Projekte

Beispielfragen:

- Wurde das Arbeitsprogramm zur Gänze umgesetzt?

○ Welche Aktivitäten konnten nicht umgesetzt bzw. abgeschlossen werden? Aus welchen Gründen?

- Wurden auch Projekte durchgeführt, die nicht im Arbeitsprogramm standen, und wenn ja, wie?

\section{OSTA Washington:}

Das Arbeitsprogramm wird nahezu zur Gänze umgesetzt. Im letzten Jahr konnte lediglich die geplante engere Zusammenarbeit mit ASciNA nicht umgesetzt werden. Im Jahresbericht findet sich eine entsprechende Begründung. Durchgeführte Projekte, die nicht im Arbeitsprogramm standen, werden ebenso wie neue Projekte gekennzeichnet. Siehe hierzu Abschnitt $\mathbf{7 . 2}$

Im Unterschied zu OSTA Peking gibt es kaum Delegationsreisen, da österreichische Forschungseinrichtungen und Unternehmen selbstständig Nordamerika bereisen können.

\section{OSTA Peking:}

Das Arbeitsprogramm wird annähernd vollständig umgesetzt, siehe hierzu Abschnitt 8.2. Abweichungen sind oftmals externen Gründen geschuldet (z.B. Alumni-Datenbank). Wesentliche Bestandteile des Arbeitsprogramms wiederholen sich auch. Dazu zählen die zeitintensive Betreuung von Delegationsreisen und die Mitwirkung an der Promotion der Calls der FFG. Aufgrund seiner eher generischen Definition lässt das Arbeitsprogramm auch Spielraum für ad-hoc Aktivitäten innerhalb der Beauftragungsprioritäten zu. Das ist positiv in dem Sinne, dass dadurch ad-hoc Opportunitäten 


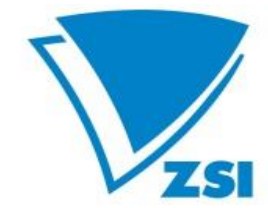

wahrgenommen werden können. Insgesamt scheint die Tätigkeit des OSTA kurzfristig ausgerichtet zu sein, Schwerpunktsetzungen jenseits der sich wiederholenden Routinen fehlen weitgehend. Die Kurzfristigkeit wird damit begründet, dass in China selbst wenig langfristig geplant wird und man sich immer wieder auf neue Akteurs Konstellationen einstellen müsse. Des Weiteren wird argumentiert, dass für langfristige Schwerpunktsetzungen nicht genügend Geld vorhanden sei. Es bestünde die Gefahr, dass man auf chinesischer Seite Erwartungen wecken würde, die Österreich dann nicht einhalten kann. Deshalb versucht man nicht zu viel Nachfrage, die unerfüllt bleiben müsste, zu generieren, sondern eine Balance mit dem Machbaren zu halten („Erwartungsmanagement“).

Das OSTA Peking schätzt, dass früher bis zu $80 \%$ seiner Tätigkeiten in die Kompetenz des BMVIT gefallen sind. Dieser Prozentsatz hat sich zwar verringert, aber die Orientierung auf das BMVIT ist immer noch sehr hoch, wenngleich man versucht, balancierter vorzugehen.

Besonders im Wissenschaftsbereich sieht das OSTA Peking noch Erweiterungsmöglichkeiten, aber der laufende Hinweis auf fehlendes Budget aus Österreich limitiert das Spektrum an Initiativsetzungen.

\section{Verwaltung der OSTA, Leitungsstruktur und Arbeitsteilung}

- Organisatorische Rahmenbedingungen in Österreich:

Beispielfragen:

○ OSTA-Lenkungsausschuss: arbeitet der Lenkungsausschuss effizient?

- Funktioniert die Kommunikation?

- Sind die Weisungen an das OSTA gut geregelt?

- Funktioniert die Abrechnungskontrolle?

- Was könnte verbessert/optimiert werden?

- Organisatorische Rahmenbedingungen im Sitzland:

Beispielfragen:

- Funktioniert das Bürogefüge?

- Was sollte in einer Geschäftsordnung genauer geregelt werden?

- Funktioniert die Buchhaltung/die Aufbereitung der Abrechnungen?

Österreich: Die Arbeit des OSTA-Lenkungsausschusses wird von den österreichischen Institutionen, die in den USA und China interviewt wurden, ebenso wie von den meisten der in Österreich befragten Stakeholdern, als wenig effizient eingestuft. Der Lenkungsausschuss wird als Addition der Ressorts wahrgenommen, aber nicht als eine gesamtkoordinierende cross-government Einrichtung. Siehe hierzu genauer Kapitel 9.

OSTA Washington: Abgesehen von kleineren Kompetenzstreitigkeiten funktioniert das Bürogefüge gut, den einzelnen Personen sind klare Aufgabenbereiche und Ressortzuständigkeiten zugeteilt und verschriftlicht. Die Botschaft wünscht sich eine Geschäftsordnung, welche die Aufteilung der Kompetenzen und Aufgaben zwischen dem OSTA und der Botschaft genauer definiert. Siehe hierzu die Abschnitte 7.6 und 7.7. Die Gebarung wurde überprüft, es konnten keine Fehler gefunden werden. Siehe hierzu Abschnitt 7.7.

OSTA Peking: Das Bürogefüge in Peking funktioniert. Als Geschäftsordnung wird das Handbuch des auswärtigen Dienstes wahrgenommen. Die Buchhaltung und Aufbereitung der Abrechnungen sind fehlerfrei. Die Abrechnungskontrolle erfolgt in Peking nach dem 4-Augenprinzip. Es wäre gut, wenn die Kanzlei der Botschaft einbezogen werden würde, aber diesbezüglich scheint die Kapazität erschöpft zu sein. Siehe hierzu Abschnitt $\mathbf{8 . 8}$ 


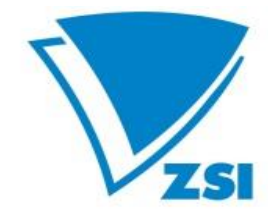

ZENTRUM FÜR SOZIALE INNOVATION

CENTRE FOR SOCIAL INNOVATION

\section{E. Betreuung von Delegationsreisen aus Österreich vor Ort}

\section{Beispielfragen:}

- Welche und wie viele Delegationsreisen wurden bzw. werden organisiert?

- In wessen Auftrag/Interesse?

- Wer hat teilgenommen?

- Wurde die Reise in Kooperation mit anderen österreichischen Einrichtungen durchgeführt (z.B. der WKÖ)?

- Welche Vernetzungsaktivitäten (z.B. RINA) wurden durchgeführt?

- Wie schätzen die Zielpersonen den Wert der Vernetzung ein?

\section{Delegationsreisen:}

Nach Nordamerika werden kaum Delegationsreisen unternommen, dafür gibt es einmal jährlich das sehr aufwändig organisierte ARIT (siehe die eigene Frage zu ARIT unter Punkt H).

In Peking wird die Betreuung der Delegationsreisen als sehr aufwändig wahrgenommen. Z.B. für die CAS-Reise wurde bis zu einem halben Jahr im Voraus gearbeitet (20-30 Leute inkl. Booklets, Organisation, Buchungen von Reisen und Hotels; Überzeugungsarbeit leisten für nicht-kooperative Institute in China ...). Außerdem ist der Servicierungsgrad für die österreichischen Teilnehmenden sehr hoch. Die Organisation der Reisen ist durch die Anti-Korruptionspolitik auch schwieriger geworden (es bedarf vieler Verbalnoten). Die chinesische Regierung greift vermehrt in die Hochschulpolitik ein und hat Kontakte der einen oder anderen Hochschule sogar schon mit der Begründung einschränkt, dass die chinesische Universität ohnehin schon genug Kontakt mit der österreichischen Botschaft hätte. Vgl. hierzu auch Abschnitt 8.4. Die Initiative von Delegationsreisen nach China geht zu $80 \%$ aus Österreich aus u.a. weil viele Besuche schon durch Abkommen manifestiert sind.

\section{Vernetzungsaktivitäten:}

Die Beantwortung der Fragen zu den Vernetzungsaktivitäten (z.B. RINA) findet sich unter Punkt G (Netzwerke)

\section{F. Informationsaufbereitung}

- Berichtswesen

Beispielfragen:

- Wie erfolgt die Übermittlung von Berichten?

- Wird das OSTA seiner Rolle gerecht?

- Newsletter

Beispielfragen:

○ Gibt es Feedback der User? Wird die Wirkung der Newsletter regelmäßig evaluiert?

- Öffentlichkeitsarbeit

Beispielfragen:

○ Welche Kanäle werden genutzt? In welchem Ausmaß?

\section{Berichtswesen:}

Die Übermittlung von Berichten erfolgt im Wesentlichen im Rahmen der Jahresberichte und der Quartalsberichte. Bei OSTA Washington werden Berichte direkt übermittelt, bei OSTA Peking laufen 


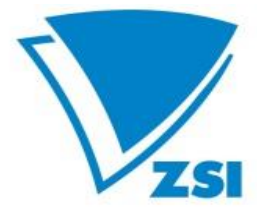

alle Berichte, die politisch (auch FTI-politisch) relevant sind, ausschließlich über die Botschaft ins BMEIA. Die Informationen müssen entweder übers BMEIA weiterverteilt, oder informell übermittelt werden. Sitzungsprotokolle zwischen MOST und BMBWF bekommt OSTA oft zuerst von MOST; hier wird ein besserer Informationskreislauf gewünscht (sowohl vom OSTA als auch zum OSTA)

\section{Newsletter:}

OSTA Peking verfasst alle 1 bis 2 Monate einen Newsletter, um die österreichische Community zu Ereignissen in China zu informieren. Die Ressorts schätzen dies mehrheitlich; Stakeholder äußern sich hingegen kritisch und meinen, die Newsletter seien zu retrospektiv sind läsen einen echten Neuigkeitscharakter vermissen.

OSTA Washington verfasst regelmäßig Berichte zu seinen Aktivitäten sowie Dossiers zu aktuellen Themen wie z.B. Cyber Security, Artificial Intelligence, Block Chain, Health oder Science Diplomacy. Diese Dossiers sind sorgfältig recherchiert und werden entsprechend geschätzt. Zentrale Stakeholder (Teilnehmende der Fokusgruppe) wünschen sich hier eine bessere Verbreitung.

\section{Öffentlichkeitsarbeit:}

In der Öffentlichkeitsarbeit kommuniziert OSTA Washington über das Netzwerk RINA, über den Blog BRIDGES sowie über Social Media (Twitter, Facebook und LinkedIn). OSTA Peking ist nicht in Social Media aktiv (Ausnahme WeChat-Gruppe für Alumni unter OeAD-Leitung); die Homepage bietet auch eher wenig Informationen.

\section{G. Netzwerke}

- RINA am OSTA Washington:

Beispielfragen:

- Wie viele Forscherinnen und Forscher sind registriert?

- Wie viele Uni-AbsolventInnen sind registriert? (Alumni)

- Gibt es einen Jahresvergleich? - steigen die Zahlen der Registrierungen?

- Wie wird das Netzwerk genutzt?

- Wie viele Aussendungen wurden 2018 gemacht?

- Wie viele Kontakte wurden 2018 vermittelt?

- Wie viele Anfragen konnten mittels RINA beantwortet werden?

- Kann das Netzwerk auch von den Mitgliedern zur direkten Interaktion mit anderen RINA Mitgliedern genutzt werden?

- Alumni-Datenbank am OSTA Peking

Beispielfragen:

- Wie viele Mitglieder hat das Alumni-Netzwerk?

- Jahresvergleich - steigen die Zahlen der Registrierungen?

- Funktioniert die Zusammenarbeit mit dem OeAD-Büro an der Fudan Universität Shanghai?

- Wie wird das Netzwerk genutzt?

- Wie viele Kontakte wurden vermittelt?

- Wie viele Aussendungen wurden gemacht? 


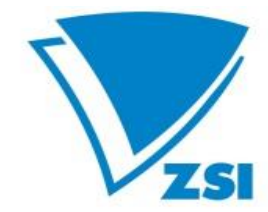

\title{
RINA
}

Bei RINA (Research and Innovation Network Austria) handelt es sich um ein sehr umfangreiches und gut gepflegtes Netzwerk, das von OSTA Washington betrieben wird. In RINA sind derzeit (Stand April 2019) insgesamt 2.960 aktive Kontakte gelistet, archivierte Kontakte sind zusätzlich noch 297 Personen. Der Schwerpunkt liegt auf Forschenden, die entweder derzeit oder in der Vergangenheit in Nordamerika tätig sind/waren. Eine genaue Beantwortung aller Beispielfragen findet sich in Abschnitt 7.5

\section{Alumni Datenbank}

Der Aufbau der Alumni-Datenbank hat sich verzögert, wobei die Ursache insbesondere in der verzögerten Übermittlung einer DSGVO-kompatiblen Datenstrukturierung durch den OeAD an das OSTA lag. In der Alumni-Datenbank, die im OSTA liegt, sind 140 Alumni erfasst. Es handelt sich bei den Alumni fast ausschließlich um chinesische Personen, die in Österreich studiert oder Kurzforschungsaufenthalte durchgeführt hatten. Details über die Nutzung der Datenbank, die über die eigentlichen Alumni-Events und social-media-Vernetzungen hinausgehen, fehlen. Eine genauere Beschreibung findet sich in Abschnitt $\mathbf{8 . 6}$

\section{H. ARIT}

- Möglichkeit zur Vernetzung über die Schwerpunktthemen hinaus

- Infoaustausch über die Entwicklungen in den USA und Österreich

- „Jobplattform“ - Information über Jobmöglichkeiten in Österreich

\begin{abstract}
ARIT wird insgesamt sehr breit kommuniziert (über RINA und BRIDGES, über Twitter, über österreichische Stellen) und wahrgenommen (war nahezu allen Befragten bekannt, viele Teilnehmende, Berichterstattung in österreichischen Medien). ARIT wird sowohl von OSTA Washington selbst als auch von vielen Stakeholdern als "jährlicher Fix- und Höhepunkt" wahrgenommen. Sowohl die interviewten Personen aus den Ressorts, als auch von den Befragten des online Surveys und die Teilnehmenden der Fokusgruppe bewerteten ARIT überwiegend positiv und bejahten sowohl die Möglichkeit zur Vernetzung über die Schwerpunktthemen hinaus als auch den intensiven Informationsaustausch über die Entwicklungen in den USA und Österreich. Weitre Informationen zu ARIT finden sich in Abschnitt 7.3

Informationen über Jobmöglichkeiten in Österreich (und Europa) bietet die jährliche Netzwerkkonferenz der European Scientific Diaspora gemeinsam mit dem EURAXESS Mentoring Programm (traditionell am Vorabend des ARIT). Auch wurde im Jahr 2018 eine Studie zu Karrierechancen und zur Unterstützung der FTI Community durch OSTA durchgeführt, die auch eine Befragung aller RINA Mitglieder inkludierte.
\end{abstract}

\section{Stakeholder}

\section{Beispielfragen:}

- Ist den Stakeholdern in Österreich das OSTA bekannt?

- Sind die Leistungen des OSTA nützlich für die Stakeholder?

- Welche Leistungen brauchen die Stakeholder zusätzlich?

- Wie beurteilen die Stakeholder den Auftritt und die Präsenz der OSTA?

- Ergänzen sich die Tätigkeiten mit anderen (Vernetzungs-)Maßnahmen? 


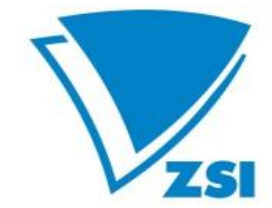

ZENTRUM FÜR SOZIALE INNOVATION

CENTRE FOR SOCIAL INNOVATION

- Wie beurteilen jene Personen, welche die Beratung in Anspruch nehmen, die Leistungen des OSTA?

○ Gibt es Verbesserungsvorschläge?

Die Frage der Bekanntheit der OSTA bei den Stakeholdern hängt in erster Linie von der Stakeholderdefinition ab. Im Hochschulbereich generell ist die Bekanntheit beider OSTA eher gering. Es gibt nur sehr vereinzelte Referenzen auf das OSTA auf den Homepages der Universitäten, der Fachhochschulen sowie der außeruniversitären Forschungseinrichtungen und auch in den Interviews wurde angemerkt, dass beide OSTA - von Einzelpersonen abgesehen - wenig systematisch bekannt sind. Bezeichnend dafür ist, dass alle österreichischen Universitäten, die zu den Fokusgruppen eingeladen waren, abgesagt haben u.a. teilweise mit der Begründung, dass sie über die OSTA wenig wüssten und damit zur Diskussion auch nicht beitragen könnten. Dies war interessanterweise bei beiden Fokusgruppen der Fall - sowohl bei Washington als auch bei Peking. Dennoch ist OSTA Washington im Hochschul- und Wissenschaftssektor sicherlich bekannter, zum einen aufgrund der längeren Geschichte, zum anderen aufgrund des breiten Netzwerkes RINA und der Verbindung zu ASciNA. Unternehmen und außeruniversitäre Forschungseinrichtungen kennen OSTA Washington nur wenig, OSTA Peking vor allem dann, wenn sie enge Verbindungen zum BMVIT oder zur FFG haben und/oder an Delegationsreisen teilgenommen haben. Bei den Agenturen und der WKÖ scheint der Bekanntheitsgrad noch am höchsten zu sein.

Der fehlende Anschluss an Kooperationsstrukturen in Österreich, die einen Outreach in die österreichische FTI-Gemeinschaft durchführen könnten, ist insgesamt als großes Defizit der OSTAStruktur festzuhalten.

In Peking führt die mangelnde Sichtbarkeit dazu, dass das OSTA weitgehend von seinen potenziellen Endkundlnnen abgeschnitten ist, und damit wenig "Rekrutierungs- bzw. Mobilisierungskompetenz" existiert. Es gibt zwar ein personalisiertes Netzwerk an Ansprechpersonen in Österreich, aber das Netzwerk ist weder systematisch strukturiert (z.B. nach Organisationen bzw. Themenfelder) noch gibt es einen systematischen Aufbau zur Entwicklung eines größeren Ansprechnetzwerkes in Österreich. Die Rekrutierung/Ansprache/Mobilisierung im Zusammenhang mit den Calls for Proposals (z.B. mit der Chinesischen Akademie der Wissenschaft oder Shanghai) funktioniert deshalb vergleichsweise gut, weil dafür die FFG die notwendigen Outreach-Arbeiten in Österreich durchführt.

Die Bekanntheit beider OSTA im Sitzland ist durchaus als zufriedenstellend einzustufen. OSTA Washington wird vonseiten amerikanischer Akteure breit wahrgenommen und geschätzt. Das OSTA Peking wird als erste Adresse für F\&E mit Österreich auf offizieller Ebene angesehen. Das OSTA Peking ist insbesondere auch für die Kontakte in und mit der chinesischen Provinz relevant. Daneben gibt es natürlich eine Vielzahl an Kontakten, die die Forschenden selbst über Konferenzen etc. etablieren und implementieren.

Bezüglich der Frage, ob die Leistungen der OSTA für die Stakeholder nützlich sind, gehen die Antworten weit auseinander. Insgesamt sind die OSTA für Erstkontakte und in diplomatischen Kontexten wichtig. Hier haben die OSTA in der Tat Türöffner Funktionen, welche sie auch gut wahrnehmen. OSTA Peking beispielsweise unterstützt die Aktivitäten der österreichischen Botschaft, wodurch sich die Austauschrunden vor Ort mit den anderen Botschaften, oftmals unter der Leitung der EU-Delegation, deutlich professionalisiert haben. In dieser Funktion ist die Präsenz auf einer Metaebene wichtig und sollte in der Weitervermittlung an spezialisiertere Einrichtungen (wie WKÖ, FFG, OeAD) bzw. direkt an potenzielle Kooperationspartner bestehen.

Weitere Informationen zum Thema "Stakeholder" finden sich in den Abschnitten $\mathbf{7 . 4}$ für Washington und 8.5 für Peking. 


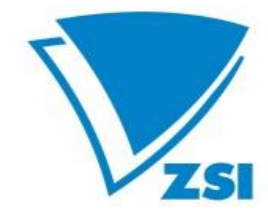

ZENTRUM FÜR SOZIALE INNOVATION

CENTRE FOR SOCIAL INNOVATION

\section{J. Abstimmung und Arbeitsteilung mit anderen Akteuren z.B. AWC der WKÖ, Open Austria in San Francisco, OeAD Kooperationsbüro Shanghai}

Beispielfragen:

- Wie funktionieren Abstimmung und Arbeitsteilung?

- Findet regelmäßiger Austausch statt?

- Gibt es gemeinsame Aktivitäten?

- Gibt es Überschneidungen der Tätigkeitsbereiche?

- Gibt es Verbesserungsmöglichkeiten, wenn ja, wie könnten diese aussehen?

\section{OSTA Washington:}

Die Kooperation mit AußenwirtschaftsCentern (AWC) werden von beiden Seiten (wie auch von der WKÖ in Österreich) als bereichernd und komplementär angesehen. Auch gibt es gemeinsame Initiativen (mit open Austria beispielsweise das gemeinsames Sommerfest im Silicon Valley) und gegenseitige operative Unterstützung (z.B. ARIT 2019 in New York). Die Zusammenarbeit mit ASciNA ist nicht ganz konfliktfrei. Auf beiden Seiten gibt es eine gewisse Furcht vor einer gegenseitigen Vereinnahmung. Gemeinsame Veranstaltungen und Aktivitäten gibt es weiterhin mit der OeAD, der Marshall Plan Foundation, dem AIT, LISA Vienna, INITS etc. OSTA Washington arbeitet mit einigen amerikanischen und auch europäischen Partnern sehr eng und sehr konstruktiv zusammen. Eine genaue Darstellung findet sich in Abschnitt 7.6

\section{OSTA Peking:}

Hier gestaltet sich die Zusammenarbeit mit den AWC konfliktreicher, da die Aufgaben im Unterschied zu OSTA Washington weniger komplementär definiert sind. Sowohl die Mehrheit der Personen aus den Ressorts als auch die meisten Stakeholder sehen die Bedarfe des Unternehmenssektors durch die AWC besser abgedeckt. Das OeAD-Büro in Shanghai wiederum hat sich sehr klar mit dem Thema internationale Bildungskooperation sowie GSK-Forschungskooperationen positioniert. Die Arbeitsteilung und Kooperation zwischen dem OSTA Peking und dem OeAD-Büro in Shanghai verläuft sehr gut. Eine genauere Darstellung findet sich in Abschnitt $\mathbf{8 . 7}$

\section{Anhänge}

\subsection{Interviewte Personen und Teilnehmende Fokusgruppen}

\section{Interviews Bundesministerien:}

- BMVIT (drei Interviews): Gernot Grimm und Alexander Unkart, Ingolf Schädler, Barbara Steiner

- BMDW (drei Interviews): Stefan Riegler und Christina Knahr, Elisabeth Rysanek, Florian Frauscher

- BMBWF (ein Interview): Heribert Buchbauer und Stefanie Nagel

- BMEIA (ein Interview): Denise Quistorp und Marcus Bergmann 


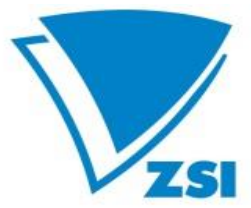

\section{Interviews Washington:}

- Clemens Mantl

- Alle MA (Simone Pötscher, Robin Tim Weis, Brittany Boykin) wurden einzeln interviewt. Tanja Ölschläger (derzeit vom BMBWF entsandt) war bei Gruppendiskussionen zusätzlich anwesend

- Stephen Ezell, information technology and innovation foundation, Washington

- Lisa Bernstein, Fulbright Washington

- Elisa Arthofer, ASciNA Chapter Washington

- Michael Friedl AußenwirtschaftsCenter New York

- Wolfgang A. Waldner, österreichischer Botschafter in Washington

- Michelle Lynch, Public Policy bei Google

- Franz Franchetti, ehemaliger Präsident vonr ASciNA

\section{Interviews Peking:}

- Philipp Agathonos und Max Pfeiler (1 Tag) sowie nur Philipp Agathonos (1/2 Tag)

- Brigitte Weiss, bmvit

- Martin Glatz (Wirtschaftsdelegierter)

- Alexandra Wagner (ÖAD Office Shanghai)

- Gabriele Gil-Feigl (Gesandte mit der Funktion der Erstzugeteilten an der Österreichischen Botschaft Peking)

\section{Sonstige Interviews Ö:}

- Markus Schweiger, Marshall-Plan Foundation

- Philipp Marxgut, ehemaliger Leiter OSTA Washington

- Andrea Höglinger FFG

\section{Teilnehmende Fokus Gruppe Washington:}

- Ralf König, FFG

- Lydia Skarits, OeAD

- Christoph Bärenreuter, FWF

- Elvira Welzig, AIT

- Georg Schneider IST Austria

- Bernhard Plunger, ÖAW

- Georg Weingartner, WKÖ

- Peter Illetschko, Der Standard 


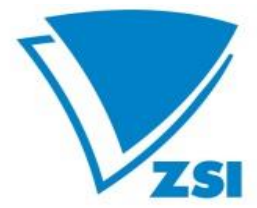

ZENTRUM FÜR SOZIALE INNOVATION

CENTRE FOR SOCIAL INNOVATION

Teilnehmende Fokus Gruppe Peking:

- Margit Haas, FFG

- Jakob Calice, OeAD

- Christoph Bärenreuter, FWF

- Sandra Schneider, AIT

- Martin Russ, Austria Tech

- Tino Terraneo, ATC

- Georg Weingartner, WKÖ

- Anton Graschopf, RFTE

- Jennifer Zhang Xiaojun, Wirtschaftsagentur Wien

\subsection{Matrix zu Wirkungsindikatoren}

\begin{tabular}{|c|c|c|c|c|}
\hline Ziel-Dimensionen & Leistungsbereiche & Output & Outcome & Impact \\
\hline \multirow[t]{3}{*}{$\begin{array}{l}\text { FTl- } \\
\text { Politikunterstützu } \\
\text { ng }\end{array}$} & $\begin{array}{l}\text { Analysen und } \\
\text { Berichte (Beiträge) } \\
\text { zu systemischen } \\
\text { Bereichen (Akteure, } \\
\text { Strategien, } \\
\text { Finanzierung etc.) }\end{array}$ & $\begin{array}{l}\text { Anzahl der erarbeiteten } \\
\text { Beiträge }\end{array}$ & \multirow{3}{*}{$\begin{array}{l}\text { Verwendung } \\
\text { und Aufgreifen } \\
\text { durch nationale } \\
\text { Verwaltungen, } \\
\text { Institutionen } \\
\text { und Akteure: } \\
\text { Anzahl der } \\
\text { Downloads von } \\
\text { Beiträgen; } \\
\text { Anzahl der } \\
\text { Empfänger von } \\
\text { Beiträgen; } \\
\text { (Zitate von } \\
\text { Beiträgen) }\end{array}$} & \multirow[t]{3}{*}{$\begin{array}{l}\text { Gesteigertes } \\
\text { Wissen über } \\
\text { Strukturen, } \\
\text { Entwicklungen } \\
\text { und Chancen im } \\
\text { Sitzland }\end{array}$} \\
\hline & $\begin{array}{l}\text { Analysen und } \\
\text { Berichte (Beiträge) } \\
\text { zu thematischen } \\
\text { Bereichen (Akteure, } \\
\text { Strategien, } \\
\text { Finanzierung etc.) }\end{array}$ & & & \\
\hline & $\begin{array}{l}\text { Herstellen von } \\
\text { Kontakten mit FTI- } \\
\text { Stakeholdern und } \\
\text { Politik }\end{array}$ & $\begin{array}{l}\text { Anzahl Kontakte mit } \\
\text { unterschiedl. } \\
\text { Institutionen vor Ort }\end{array}$ & & \\
\hline $\begin{array}{l}\text { Unterstützung } \\
\text { bilateraler } \\
\text { Kooperationen }\end{array}$ & $\begin{array}{l}\text { Vorbereitung FTI- } \\
\text { politische } \\
\text { Kooperationsvereinb } \\
\text { arungen } \\
\text { (MoU, Lol etc.) }\end{array}$ & $\begin{array}{l}\text { Anzahl vorbereiteter } \\
\text { Kooperationvereinbarun } \\
\text { gen }\end{array}$ & $\begin{array}{l}\text { weiterführende } \\
\text { Aktivitäten } \\
\text { nationaler FTI- } \\
\text { Politik im } \\
\text { Zielland; } \\
\text { weiterführende } \\
\text { Aktivitäten } \\
\text { nationaler FTI- } \\
\text { Politik in } \\
\text { Österreich }\end{array}$ & $\begin{array}{l}\text { Nachhaltige } \\
\text { Verankerung } \\
\text { österreichischer } \\
\text { Akteure im } \\
\text { Sitzland; } \\
\text { Erhöhte Anreize } \\
\text { für Akteure im } \\
\text { Sitzland mit } \\
\text { nationalen }\end{array}$ \\
\hline
\end{tabular}




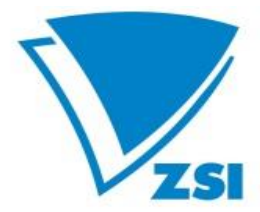

\begin{tabular}{|c|c|c|c|c|}
\hline Ziel-Dimensionen & Leistungsbereiche & Output & Outcome & Impact \\
\hline & $\begin{array}{l}\text { Unterstützung der } \\
\text { Errichtung bi- } \\
\text { lateraler FTI- } \\
\text { Programme } \\
\text { zwischen Österreich } \\
\text { und Sitzland }\end{array}$ & $\begin{array}{l}\text { Anzahl eingerichtete bi- } \\
\text { laterale Programme } \\
\text { (evtl. Anzahl Calls, } \\
\text { Fördervolumina etc.) }\end{array}$ & $\begin{array}{l}\text { Erhöhung der } \\
\text { Anzahl der } \\
\text { Förderfälle im } \\
\text { Sitzland }\end{array}$ & $\begin{array}{l}\text { Akteuren zu } \\
\text { kooperieren }\end{array}$ \\
\hline & Delegationsreisen & $\begin{array}{l}\text { Anzahl von } \\
\text { Delegationsreisen, } \\
\text { Anzahl Teilnehmerlnnen } \\
\text { nach Themenbereichen }\end{array}$ & $\begin{array}{l}\text { weiterführende } \\
\text { Aktivitäten der } \\
\text { FTI-Community } \\
\text { im Zielland; } \\
\text { weiterführende } \\
\text { Aktivitäten der } \\
\text { FTI-Community } \\
\text { in Österreich }\end{array}$ & \\
\hline & $\begin{array}{l}\text { Unterstützung von } \\
\text { nationalen FTI- } \\
\text { Programmen }\end{array}$ & $\begin{array}{l}\text { Anzahl der Kontakte mit } \\
\text { nationalen } \\
\text { Intermediären, Anzahl } \\
\text { der über OSTA } \\
\text { beworbene/betreute/un } \\
\text { terstützte Programme }\end{array}$ & $\begin{array}{l}\text { Erhöhung der } \\
\text { Anzahl der } \\
\text { Förderfälle im } \\
\text { Sitzland }\end{array}$ & \\
\hline \multirow[t]{3}{*}{$\begin{array}{l}\text { Unterstützung } \\
\text { des Wissens- und } \\
\text { Know-How- } \\
\text { Transfers }\end{array}$} & $\begin{array}{l}\text { Unterstützung von } \\
\text { Kooperationen auf } \\
\text { institutioneller } \\
\text { Ebene }\end{array}$ & $\begin{array}{l}\text { erfolgreiche Vermittlung } \\
\text { von } \\
\text { Forschenden/Unterneh } \\
\text { men in beide } \\
\text { Richtungen: } \\
\text { Anzahl der initiierten } \\
\text { "neuen" Kooperationen }\end{array}$ & $\begin{array}{l}\text { Erhöhung der } \\
\text { Zahl } \\
\text { strategischer } \\
\text { Forschungs- } \\
\text { und } \\
\text { Kooperationspr } \\
\text { ojekte } \\
\text { nationaler } \\
\text { Akteure im } \\
\text { Sitzland: } \\
\text { Anzahl der } \\
\text { initiierten oder } \\
\text { unterstützten } \\
\text { F\&l-Projekte; } \\
\text { Anzahl der } \\
\text { Lizensierungsfä } \\
\text { lle }\end{array}$ & $\begin{array}{l}\text { Kompetenzerwe } \\
\text { rb, erhöhte } \\
\text { Wettbewerbsfä } \\
\text { higkeit, Beitrag } \\
\text { zu Entwicklung } \\
\text { von Lösungen in } \\
\text { strategischen/ } \\
\text { gesellschaftlich } \\
\text { relevanten } \\
\text { Bereichen }\end{array}$ \\
\hline & \multirow[t]{2}{*}{$\begin{array}{l}\text { Unterstüzung } \\
\text { Technologietranfer } \\
\text { in strategischen } \\
\text { Technologiefeldern }\end{array}$} & $\begin{array}{l}\text { Identifikation zentraler } \\
\text { Player }\end{array}$ & \multirow{2}{*}{$\begin{array}{l}\text { Patente, } \\
\text { Forschungsleist } \\
\text { ungen auf Basis } \\
\text { kooperativer } \\
\text { Projekte }\end{array}$} & \\
\hline & & $\begin{array}{l}\text { Anzahl organisierter } \\
\text { Workshops, } \\
\text { Veranstaltungen }\end{array}$ & & \\
\hline
\end{tabular}




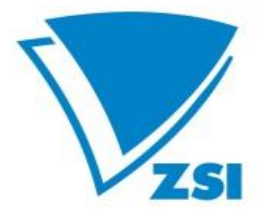

\begin{tabular}{|c|c|c|c|c|}
\hline Ziel-Dimensionen & Leistungsbereiche & Output & Outcome & Impact \\
\hline & & $\begin{array}{l}\text { Anzahl organisierter FTI- } \\
\text { Organisations- und } \\
\text { Firmenbesuche }\end{array}$ & & \\
\hline \multirow[t]{2}{*}{$\begin{array}{l}\text { Betreuung } \\
\text { österreichischer } \\
\text { Forschungseinrich } \\
\text { tungen und } \\
\text { Forscher/innen }\end{array}$} & Netzwerkpflege & $\begin{array}{l}\text { Anzahl der } \\
\text { Netzwerkmitglieder } \\
\text { (Sitz, Typ Institution etc.) }\end{array}$ & $\begin{array}{l}\text { Weiterentwickl } \\
\text { ung des } \\
\text { Netzwerkes; } \\
\text { Erschließung } \\
\text { neuer } \\
\text { Gruppen/ } \\
\text { Regionen }\end{array}$ & $\begin{array}{l}\text { Nachhaltige } \\
\text { Netzwerke }\end{array}$ \\
\hline & $\begin{array}{l}\text { Service- und } \\
\text { Beratungsleistungen }\end{array}$ & $\begin{array}{l}\text { Anzahl von } \\
\text { Beratungsleistungen } \\
\text { (nach Bereich, Typ von } \\
\text { Institution etc.) }\end{array}$ & $\begin{array}{l}\text { Kunden- } \\
\text { zufriedenheit } \\
\text { (Befragung); } \\
\text { Enabling und } \\
\text { nachhaltige } \\
\text { Verankerung } \\
\text { der } \\
\text { Forschenden } \\
\text { im Sitzland }\end{array}$ & $\begin{array}{l}\text { Nachhaltige } \\
\text { F\&E- } \\
\text { Kooperationen, } \\
\text { Wissentransfer } \\
\text { nach Österreich }\end{array}$ \\
\hline \multirow[t]{2}{*}{$\begin{array}{l}\text { Betreuung von } \\
\text { Forschungseinrich } \\
\text { tungen und } \\
\text { Forscher/innen } \\
\text { im Sitzland mit } \\
\text { Interesse an } \\
\text { Österreich }\end{array}$} & Netzwerkpflege & $\begin{array}{l}\text { Anzahl der } \\
\text { Netzwerkmitglieder } \\
\text { (Sitz, Typ Institution etc.) }\end{array}$ & $\begin{array}{l}\text { Weiterentwickl } \\
\text { ung des } \\
\text { Netzwerkes; } \\
\text { Erschließung } \\
\text { neuer } \\
\text { Gruppen/ } \\
\text { Regionen }\end{array}$ & $\begin{array}{l}\text { Nachhaltige } \\
\text { Netzwerke }\end{array}$ \\
\hline & $\begin{array}{l}\text { Service- und } \\
\text { Beratungsleistungen }\end{array}$ & $\begin{array}{l}\text { Anzahl von } \\
\text { Beratungsleistungen } \\
\text { (nach Bereich, Typ von } \\
\text { Institution etc.) }\end{array}$ & $\begin{array}{l}\text { Kundenzufried } \\
\text { enheit } \\
\text { (Befragung); } \\
\text { Enabling und } \\
\text { nachhaltige } \\
\text { Verankerung } \\
\text { der } \\
\text { Forschenden in } \\
\text { Österreich }\end{array}$ & $\begin{array}{l}\text { Nachhaltige } \\
\text { F\&E- } \\
\text { Kooperationen, } \\
\text { Wissentransfer } \\
\text { nach Österreich }\end{array}$ \\
\hline \multirow{2}{*}{$\begin{array}{l}\text { Branding des } \\
\text { österreichischen } \\
\text { FTI-Standortes } \\
\text { erhöhen }\end{array}$} & $\begin{array}{l}\text { Vermarktung } \\
\text { österreichischer } \\
\text { Technologien }\end{array}$ & $\begin{array}{l}\text { Anzahl von Messen und } \\
\text { Veranstaltungteilnahme } \\
\mathrm{n}\end{array}$ & \multirow{2}{*}{$\begin{array}{l}\text { Verwendung } \\
\text { und Aufgreifen } \\
\text { durch } \\
\text { Verwaltungen, } \\
\text { Institutionen } \\
\text { und Akteure im } \\
\text { Sitzland }\end{array}$} & \multirow{2}{*}{$\begin{array}{l}\text { Erhöhte } \\
\text { Sichtbarkeit } \\
\text { Österreichs im } \\
\text { Sitzland; } \\
\text { Gesteigerstes } \\
\text { Interesse an } \\
\text { Kooperationen, } \\
\text { Investitionen, } \\
\text { Aufenthalten }\end{array}$} \\
\hline & $\begin{array}{l}\text { Veranstaltungen und } \\
\text { Events }\end{array}$ & $\begin{array}{l}\text { Anzahl der } \\
\text { durchgeführten } \\
\text { Veranstaltungen und } \\
\text { Events die dem Branding } \\
\text { u. Vermarktung dienen; }\end{array}$ & & \\
\hline
\end{tabular}




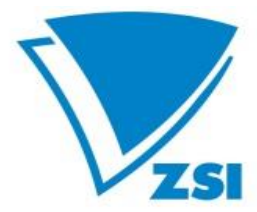

\begin{tabular}{|c|c|c|c|c|}
\hline Ziel-Dimensionen & Leistungsbereiche & Output & Outcome & Impact \\
\hline & & $\begin{array}{l}\text { Anzahl der } \\
\text { TeilnehmerInnen }\end{array}$ & & $\begin{array}{l}\text { mit und in } \\
\text { Österreich }\end{array}$ \\
\hline & $\begin{array}{l}\text { Informationsbereitst } \\
\text { ellung über } \\
\text { österreichisches } \\
\text { Forschungs- und } \\
\text { Innovationssystem }\end{array}$ & $\begin{array}{l}\text { Anzahl der } \\
\text { beantworteten Anfragen }\end{array}$ & & \\
\hline \multirow[t]{2}{*}{$\begin{array}{l}\text { EU und } \\
\text { multilaterale } \\
\text { Kooperationen } \\
\text { ausbauen }\end{array}$} & $\begin{array}{l}\text { Uneterstützung der } \\
\text { Einrichtung, } \\
\text { Bewerbung und } \\
\text { Betreuung } \\
\text { europäischer } \\
\text { Programme mit } \\
\text { nationaler } \\
\text { Beteiligung }\end{array}$ & $\begin{array}{l}\text { Anzahl der Kontakte mit } \\
\text { nationalen } \\
\text { Intermediären, Anzahl } \\
\text { der über OSTA } \\
\text { beworbene/betreute/un } \\
\text { terstützte Programme }\end{array}$ & $\begin{array}{l}\text { Erhöhung der } \\
\text { Anzahl der } \\
\text { Förderfälle im } \\
\text { Sitzland über } \\
\text { EU- und } \\
\text { multilaterale } \\
\text { Programme }\end{array}$ & \multirow{2}{*}{$\begin{array}{l}\text { Erhöhter } \\
\text { Wirkungsradius } \\
\text { der } \\
\text { östereichischen } \\
\text { Aktivitäten im } \\
\text { Sitzland durch } \\
\text { vernetztes } \\
\text { Auftreten } \\
\text { innerhalb } \\
\text { europäischer } \\
\text { Strukturen }\end{array}$} \\
\hline & $\begin{array}{l}\text { Vernetzung mit } \\
\text { internationalen } \\
\text { Vernetzungsbüros } \\
\text { anderer Staaten }\end{array}$ & $\begin{array}{l}\text { Anzahl der Kontakte mit } \\
\text { entsprechenden } \\
\text { Einrichtungen }\end{array}$ & $\begin{array}{l}\text { Know-How- } \\
\text { Gewinn }\end{array}$ & \\
\hline \multirow[t]{3}{*}{$\begin{array}{l}\text { Öffentlichkeitsarb } \\
\text { eit des OSTA }\end{array}$} & Web-Präsenz & $\begin{array}{l}\text { Anzahl der Updates auf } \\
\text { der Website pro Jahr; } \\
\text { Anzahl der Twitter, } \\
\text { Instragamm etc. Posts } \\
\text { pro Jahr }\end{array}$ & $\begin{array}{l}\text { Log-Protokoll; } \\
\text { Anzahl der } \\
\text { Clicks, Likes, } \\
\text { Verweildauer } \\
\text { etc. }\end{array}$ & \multirow{3}{*}{$\begin{array}{l}\text { erhöhte } \\
\text { Sichtbarkeit des } \\
\text { OSTA in als } \\
\text { Akteur in der } \\
\text { Nationalen FTI- } \\
\text { Community } \\
\text { sowie in jener } \\
\text { des Sitzlandes; } \\
\text { Hohe } \\
\text { Bekanntheit der } \\
\text { Leistungsbereic } \\
\text { he des OSTA; } \\
\text { Erschließung } \\
\text { neuer } \\
\text { KundInnen- } \\
\text { Gruppen }\end{array}$} \\
\hline & Veranstaltungen & $\begin{array}{l}\text { Anzahl der } \\
\text { durchgeführten } \\
\text { Veranstaltungen und } \\
\text { Events die dem Branding } \\
\text { u. Vermarktung dienen; } \\
\text { Anzahl der } \\
\text { TeilnehmerInnen }\end{array}$ & \multirow[t]{2}{*}{$\begin{array}{l}\text { Verwendung } \\
\text { und Aufgreifen } \\
\text { durch } \\
\text { Verwaltungen, } \\
\text { Institutionen } \\
\text { und Akteure im } \\
\text { Sitzland und } \\
\text { Österreich }\end{array}$} & \\
\hline & $\begin{array}{l}\text { Aktive Vermarktung } \\
\text { über } \\
\text { Multiplikatoreinricht } \\
\text { ungen, Intermediäre } \\
\text { und Institutionen }\end{array}$ & $\begin{array}{l}\text { Anzahl der Kontakte mit } \\
\text { entsprechenden } \\
\text { Einrichtungen }\end{array}$ & & \\
\hline
\end{tabular}




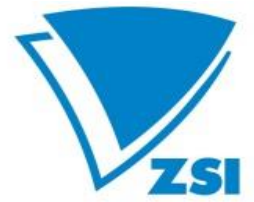

ZENTRUM FÜR SOZIALE INNOVATION

CENTRE FOR SOCIAL INNOVATION

\subsection{Ergebnisse Online Survey - Washington}

Abbildung 14: Aktivtäten von Hochschulen und Forschungseinrichtungen in Washington; $n=53$

Ich absolvier(t)e einen Forschungsaufenthalt in den USA.

Ich bin/war an einem gemeinsamen Forschungsprojekt mit amerikanischen Partnern beteiligt.

Ich bin an meiner Institution mit der Anbahnung von Forschungsprojekten bzw. Innovationsprojekten in den USA befasst.

Ich bin/war an einem gemeinsamen Forschungsprojekt mit österreichischen Partnern beteiligt.

Anderes

Ich absolvier(t)e einen Forschungsaufenthalt in Österreich.

Ich bin an meiner Institution mit der Anbahnung von Austauschaktivitäten österreichischer Forschender in Richtung USA befasst.

Ich bin an meiner Institution mit der Anbahnung von Forschungsprojekten bzw. Innovationsprojekten in Österreich befasst.

Ich bin/war an einem gemeinsamen Innovationsprojekt mit österreichischen Partnern beteiligt.

Ich bin/war an einem gemeinsamen

Innovationsprojekt mit amerikanischen Partnern beteiligt.

Ich bin an meiner Institution mit der Anbahnung von Austauschaktivitäten amerikanischer Forschender in Richtung Österreich befasst.

Meine Institution ist mit dem Aufbau kollaborativer Forschungs- und Innovationsinfrastrukturen mit österreichischer und US-amerikanischer..

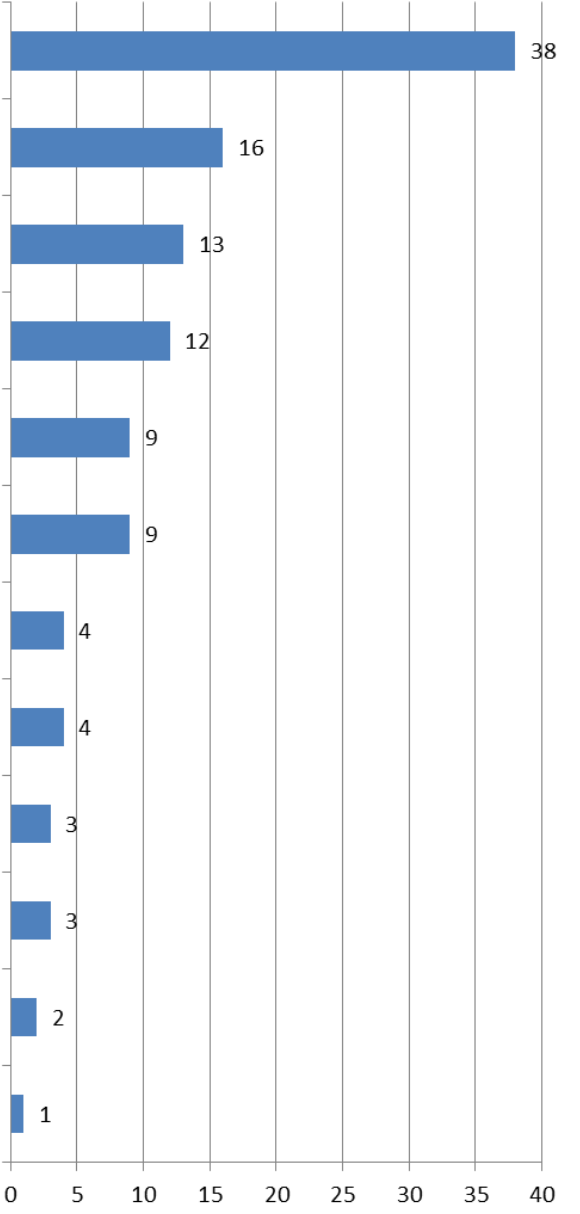

Quelle: JOANNEUM RESEARCH 


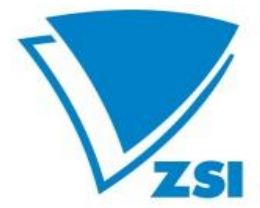

ZENTRUM FÜR SOZIALE INNOVATION

CENTRE FOR SOCIAL INNOVATION

Abbildung 15: Land des aktuellen Aufenthaltes; $N=73$

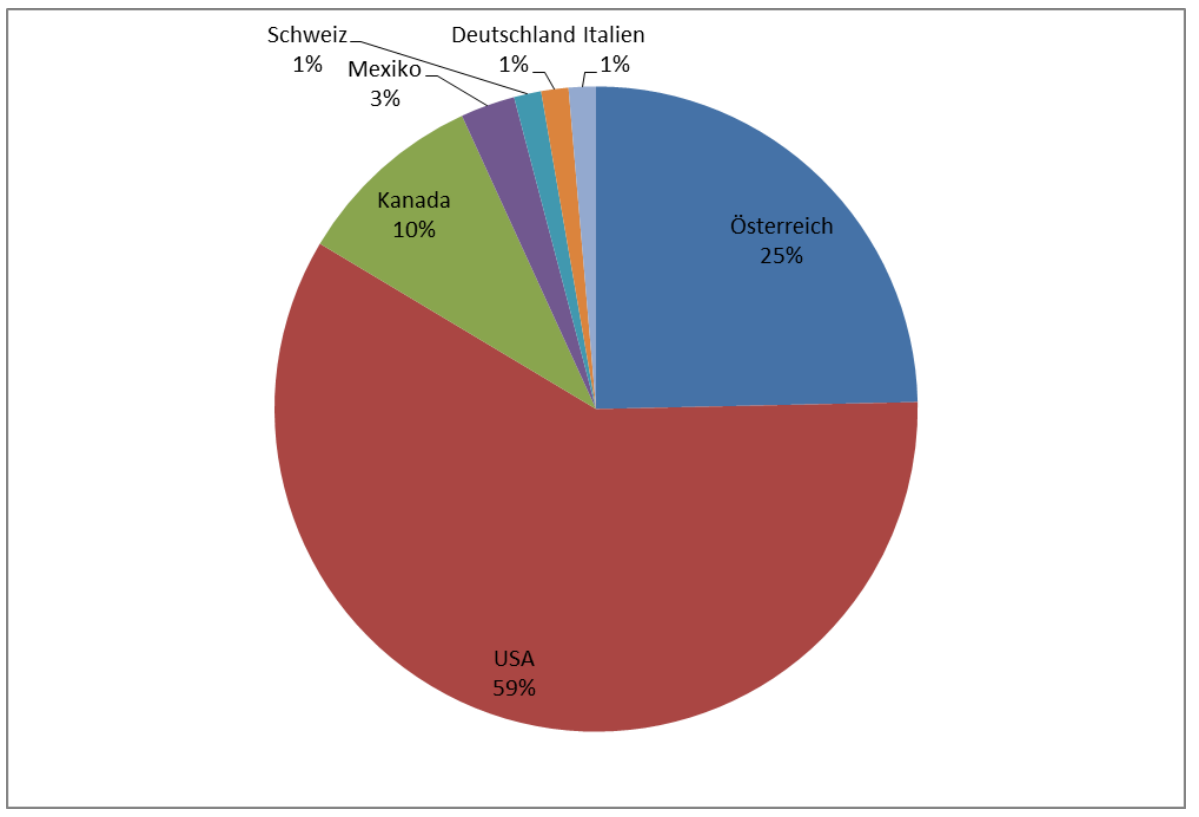

Quelle: JOANNEUM RESEARCH

Abbildung 16: Kontakt OSTA; Skala=Anzahl; $\mathrm{N}=72$

Ich war niemals in direktem Kontakt mit dem OSTA Washington und habe auch keine Leistungen in Anspruch genommen.

Ich stand/stehe in sporadischem Kontakt mit dem OSTA Washington DC.

Ich stehe in regelmäßigem Austausch mit dem OSTA Washington DC bzw. seinen Netzwerken und werde laufend über Aktivitäten informiert.

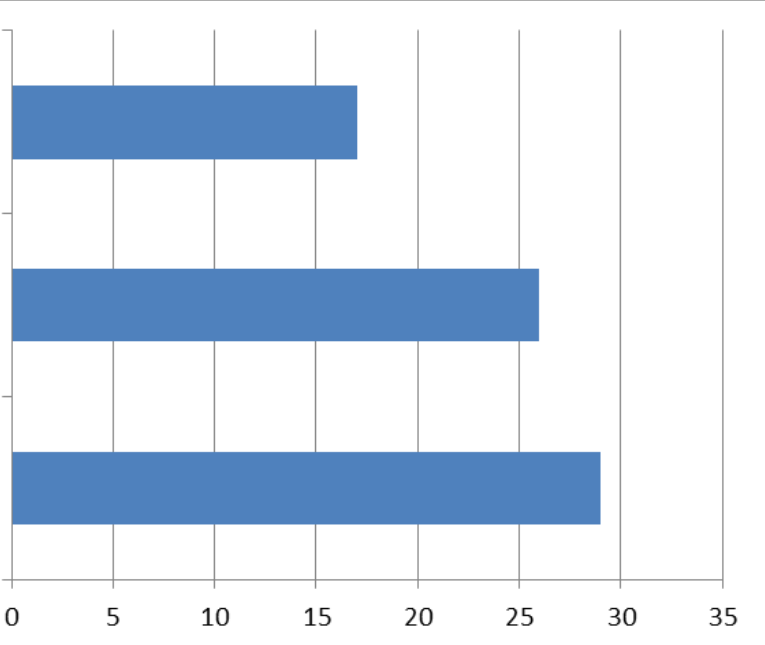

Quelle: JOANNEUM RESEARCH 


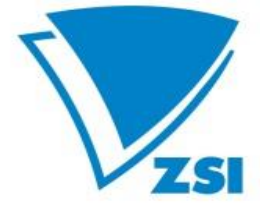

ZENTRUM FÜR SOZIALE INNOVATION

CENTRE FOR SOCIAL ININOVATION

Abbildung 17: Bekanntheit RINA\&ASciNA; Skala=Anzahl; N=73

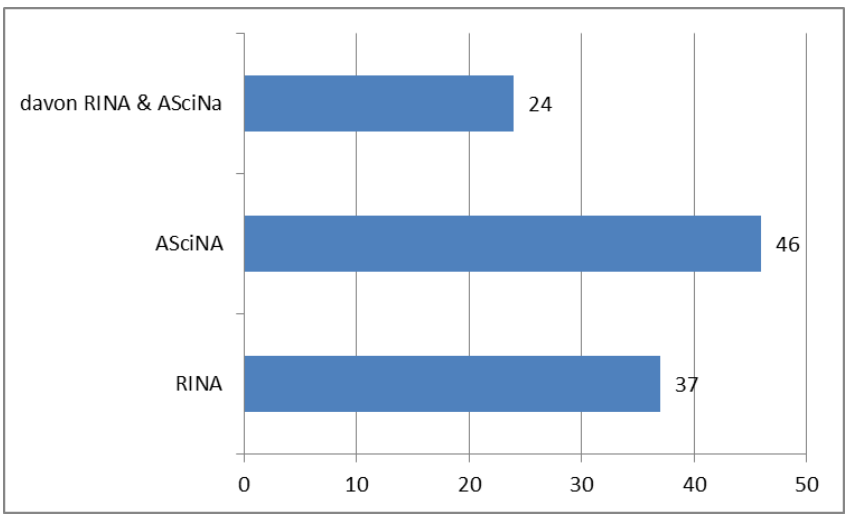

Quelle: JOANNEUM RESEARCH

Abbildung 18: Wie haben Sie von der Existenz des OSTA Washington erfahren?; Skala=Anzahl; N=72

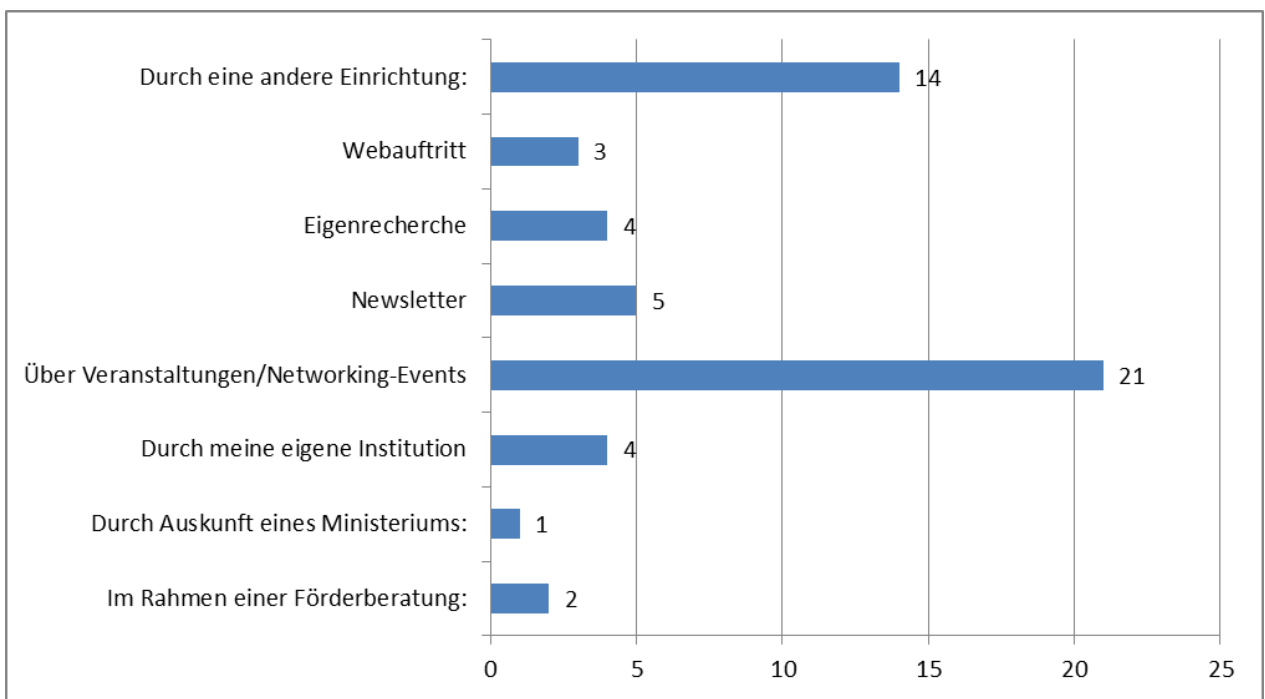

Quelle: JOANNEUM RESEARCH

Hier wurden unter „Andere Einrichtung“ mehrheitlich ASciNA, die Botschaft sowie einmal die Schrödinger Fellowship genannt. 


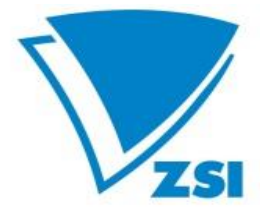

ZENTRUM FÜR SOZIALE INNOVATION

CENTRE FOR SOCIAL ININOVATION

Abbildung 19: Bitte beurteilen Sie die Relevanz folgender Aktivitätsbereiche des OSTA Washington aus Ihrer

Perspektive; Skala=Anzahl; N=Itemabhängig

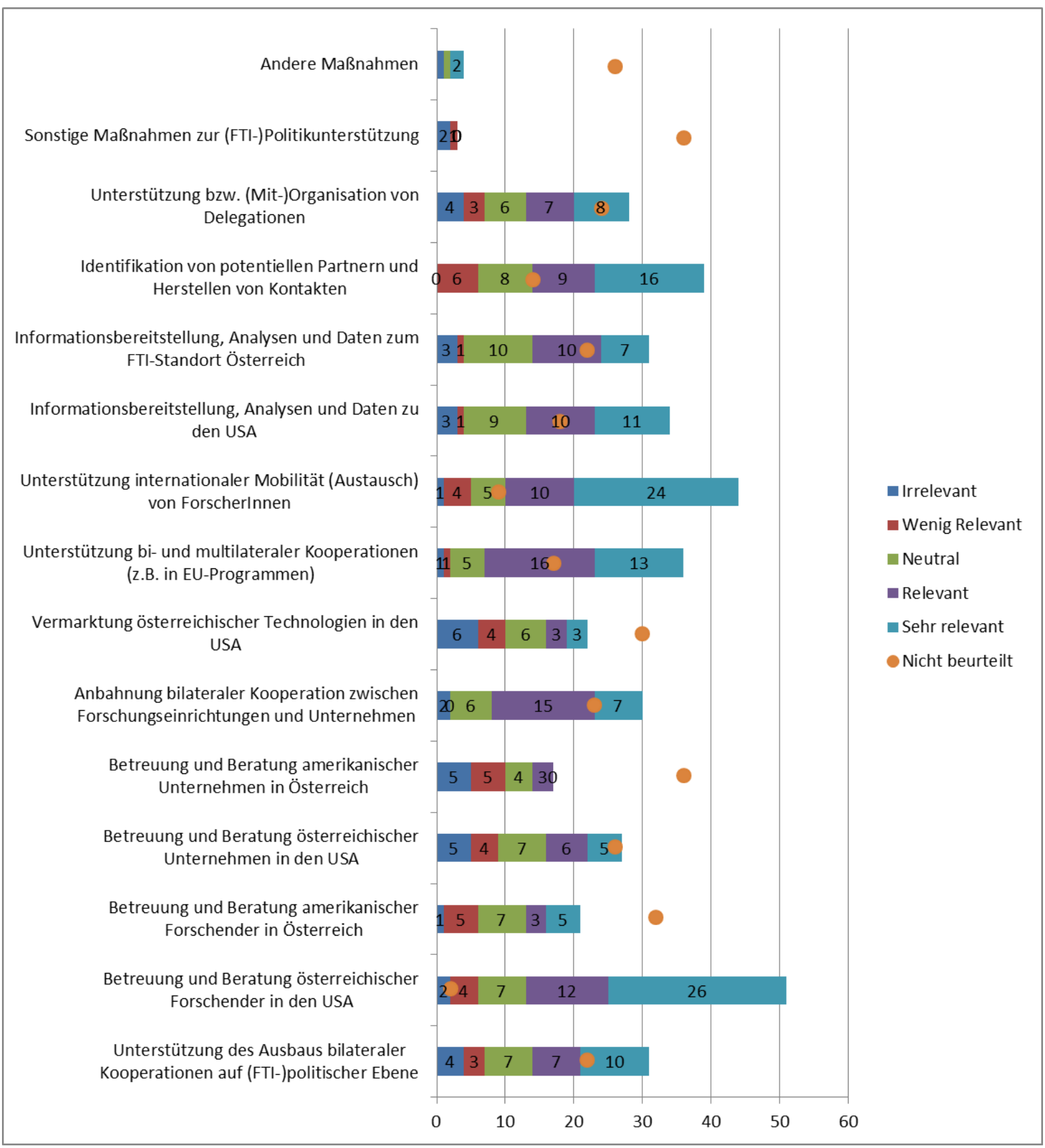

Quelle: JOANNEUM RESEARCH 


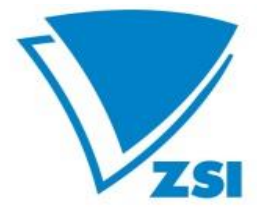

ZENTRUM FÜR SOZIALE INNOVATION

CENTRE FOR SOCIAL ININOVATION

Abbildung 20: Wie zufrieden sind/waren Sie mit den entsprechenden Leistungen?; Skala=Anzahl; N=Itemabhängig

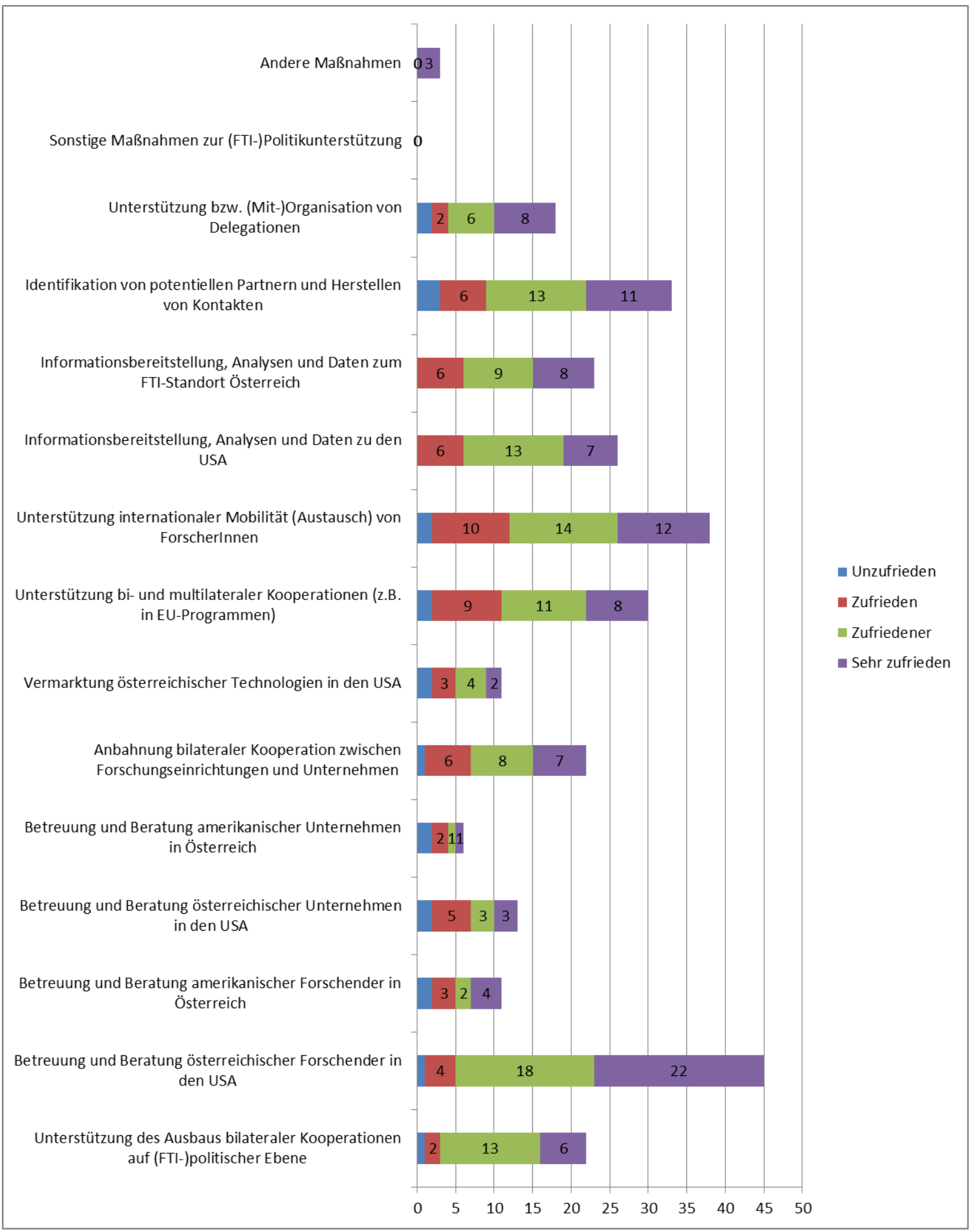

Quelle: JOANNEUM RESEARCH 


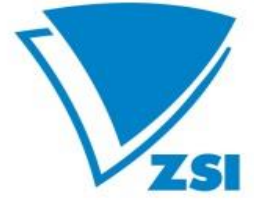

ZENTRUM FÜR SOZIALE INNOVATION

CENTRE FOR SOCIAL ININOVATION

Abbildung 21: Haben Sie bereits Leistungen des RINA-Netzwerkes in Anspruch genommen?; N=36

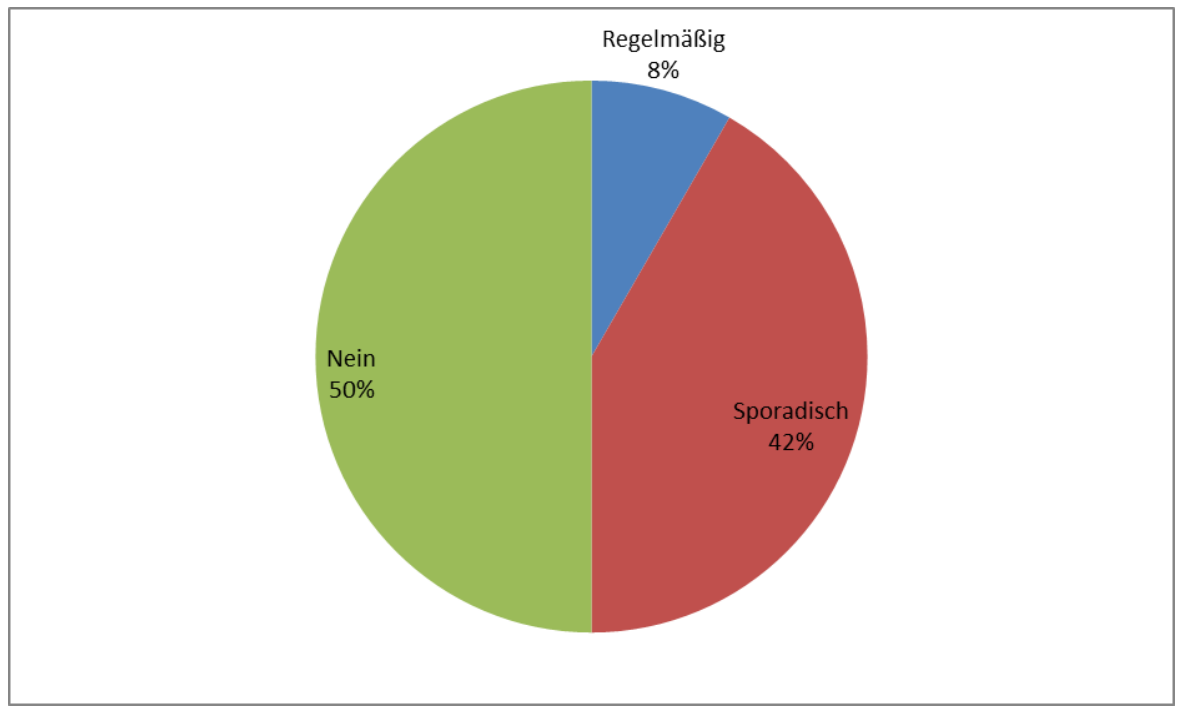

Quelle: JOANNEUM RESEARCH 


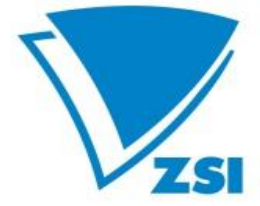

ZENTRUM FÜR SOZIALE INNOVATION

CENTRE FOR SOCIAL ININOVATION

Abbildung 22: Welche der folgenden konkreten Leistungen haben Sie vom RINA Netzwerk in Anspruch genommen?; $\mathrm{N}=$ =Itemabhängig

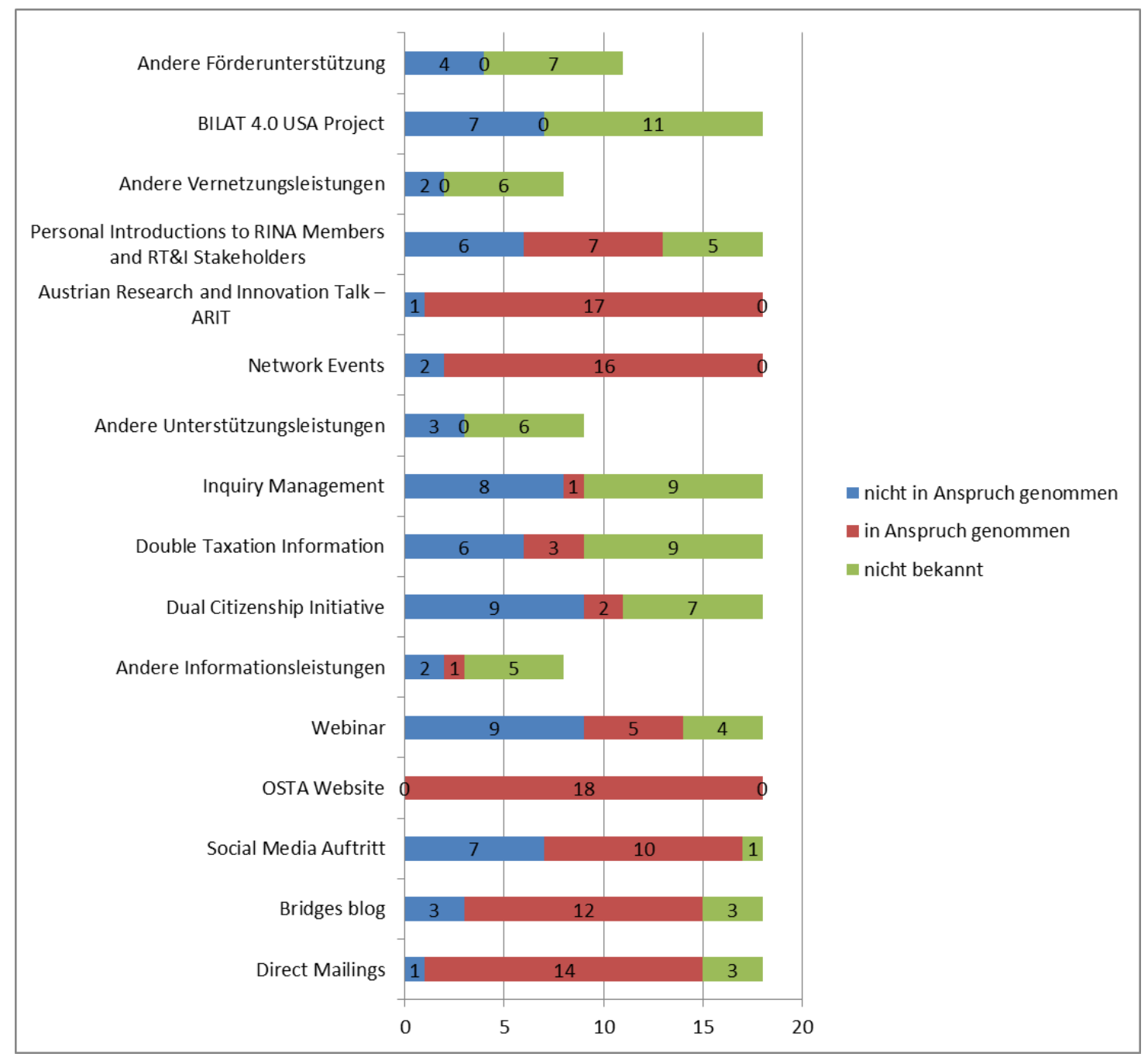

Quelle: JOANNEUM RESEARCH 


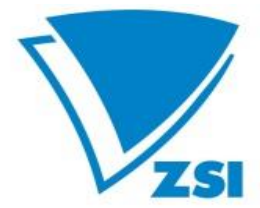

ZENTRUM FÜR SOZIALE INNOVATION

CENTRE FOR SOCIAL ININOVATION

Abbildung 23: Haben Sie bereits Leistungen des ASCinA Netzwerkes in Anspruch genommen?; N=46

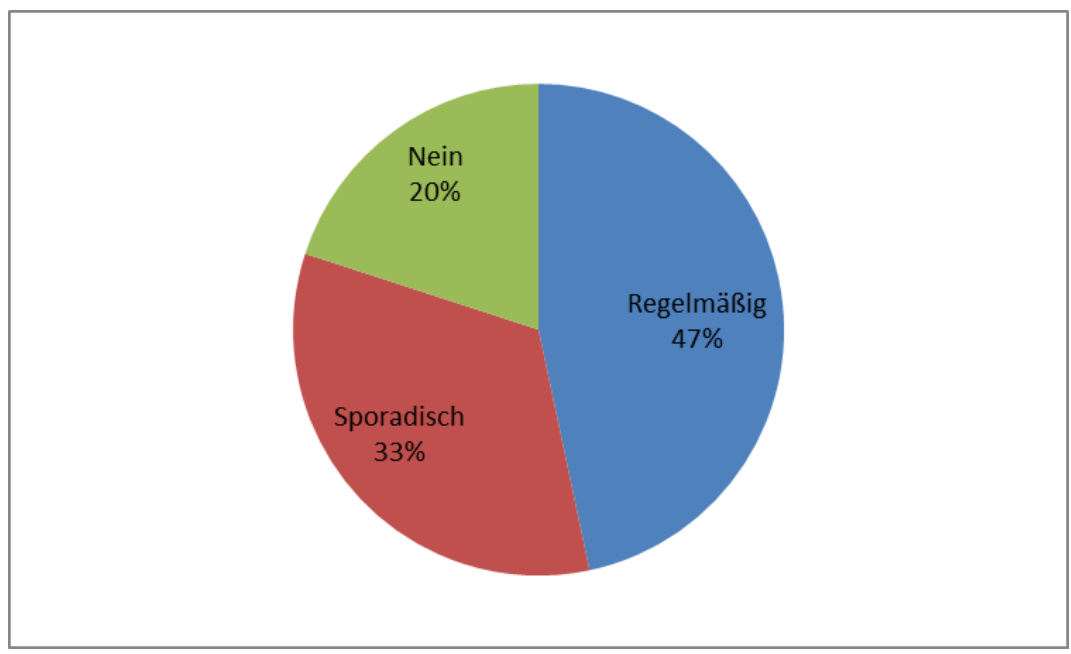

Quelle: JOANNEUM RESEARCH

Abbildung 24: Welche der folgenden konkreten Leistungen haben Sie vom ASciNA Netzwerk in Anspruch genommen?; $\mathrm{N}=36$

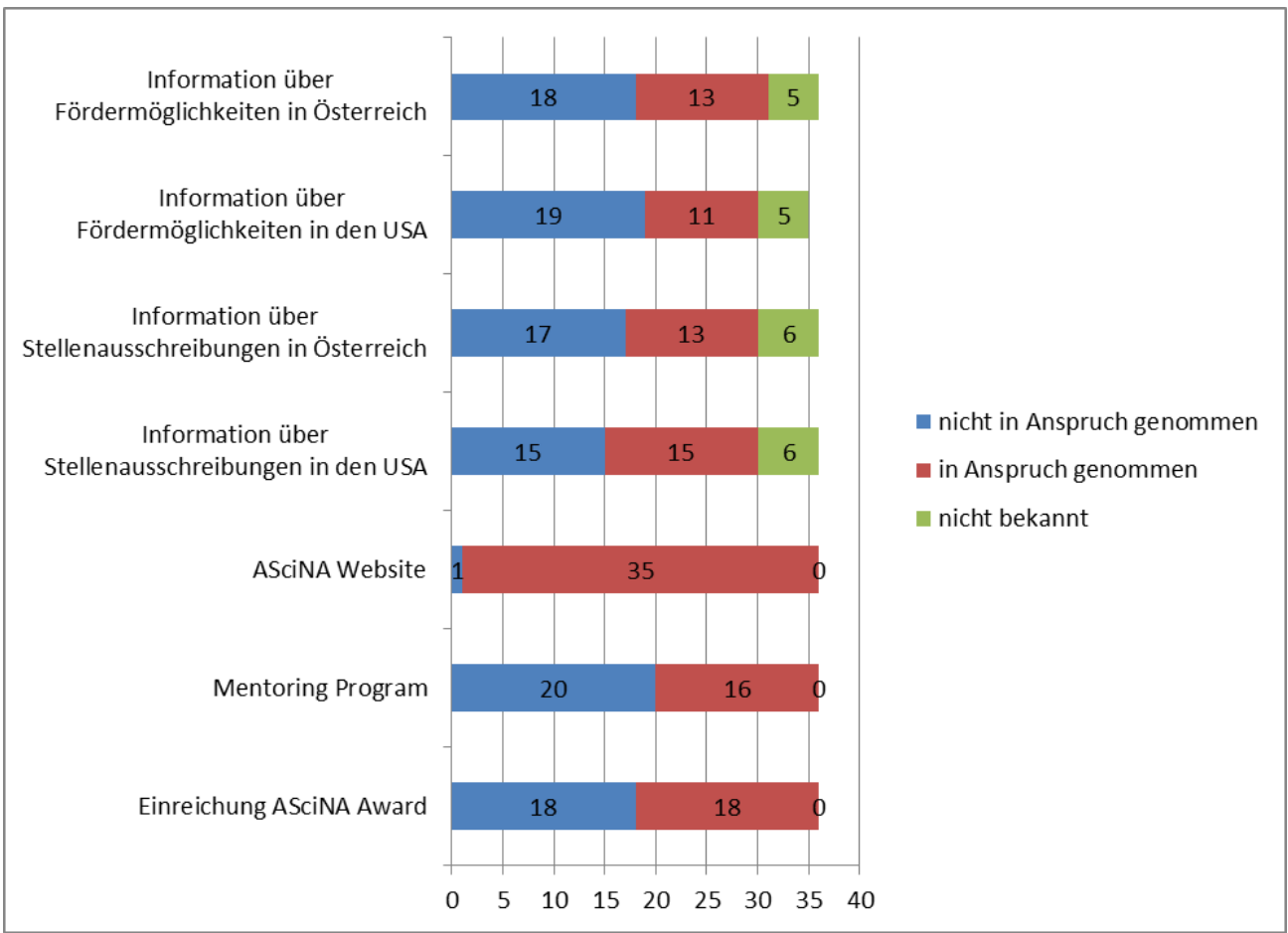

Quelle: JOANNEUM RESEARCH 


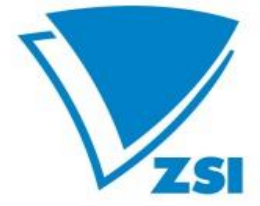

ZENTRUM FÜR SOZIALE INNOVATION

CENTRE FOR SOCIAL ININOVATION

Abbildung 25: Wie zufrieden sind Sie mit den entsprechenden Leistungen des ASciNA-Netzwerkes?; Skala=Anzahl; $\mathrm{N}=$ Itemabhängig

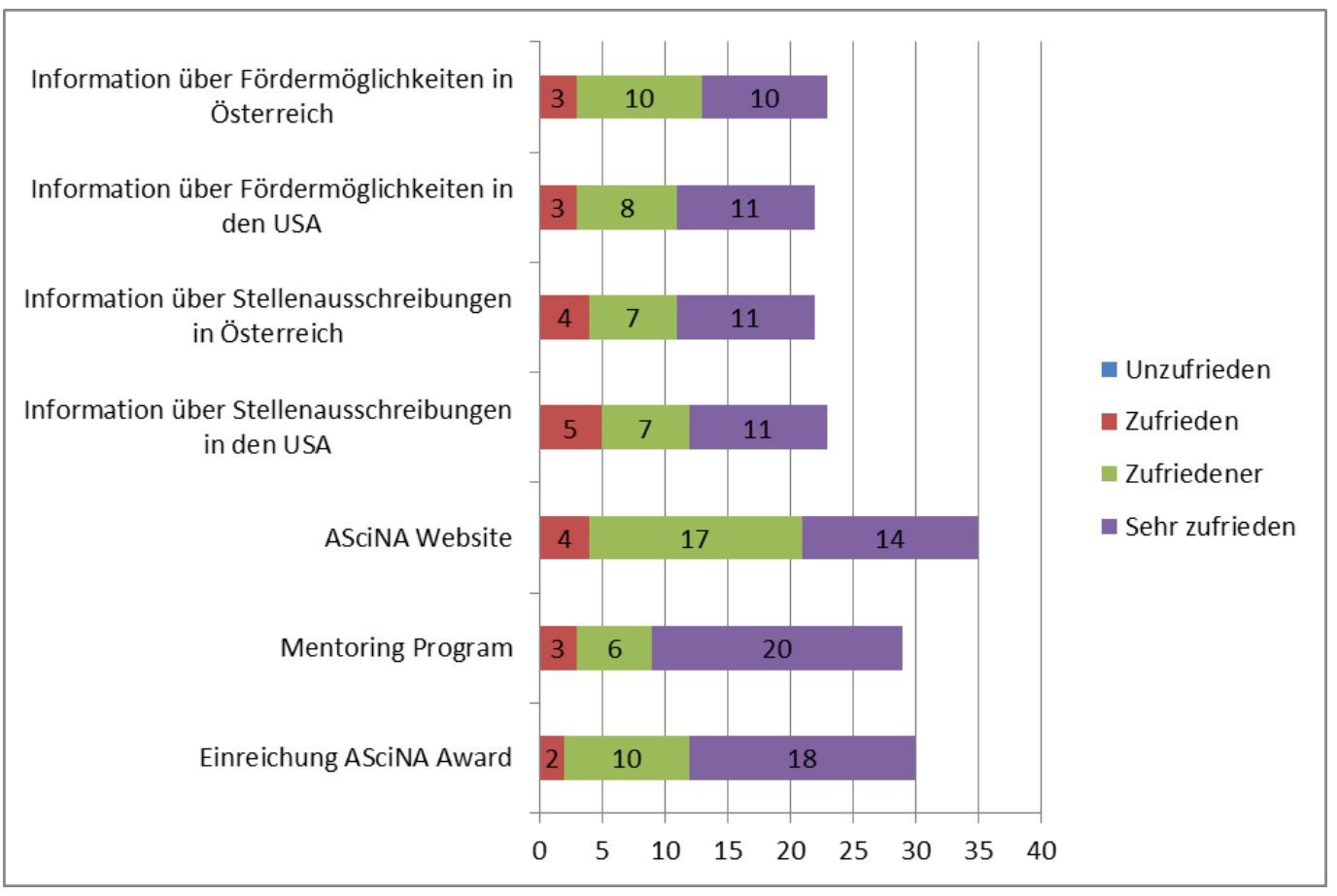

Quelle: JOANNEUM RESEARCH

Abbildung 26: War OSTA Washington die wichtigste Unterstützung/Informationsquelle, die Sie als Grundlage für Ihre Aktivitäten herangezogen haben?; $\mathrm{N}=64$

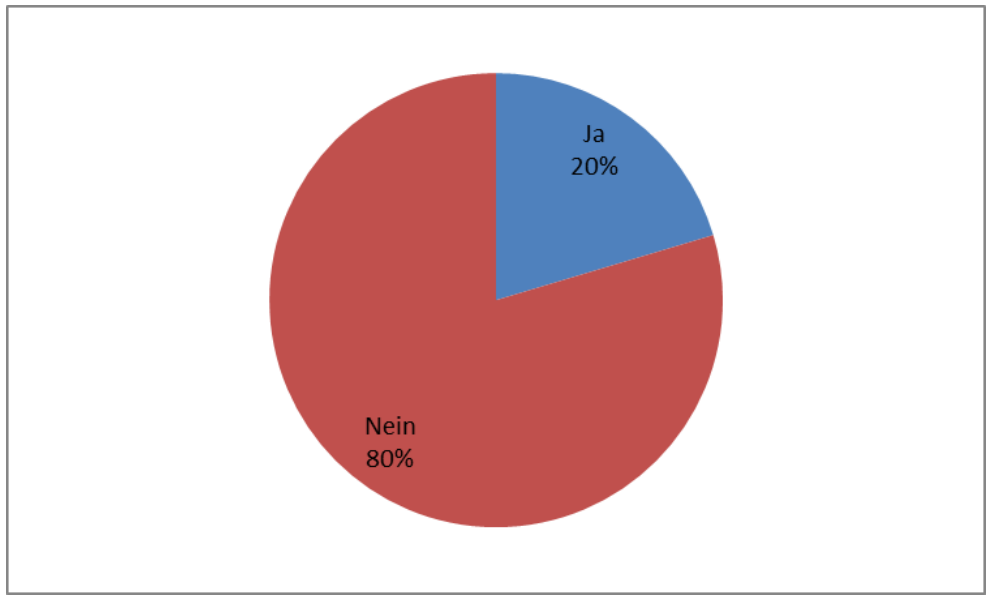

Quelle: JOANNEUM RESEARCH 


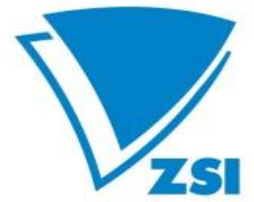

ZENTRUM FÜR SOZIALE INNOVATION

CENTRE FOR SOCIAL ININOVATION

Abbildung 27: Auf welche anderen Unterstützungsleistungen, Programme oder Akteure haben Sie zurückgegriffen?;

Skala=Anzahl; N=Itemabhängig

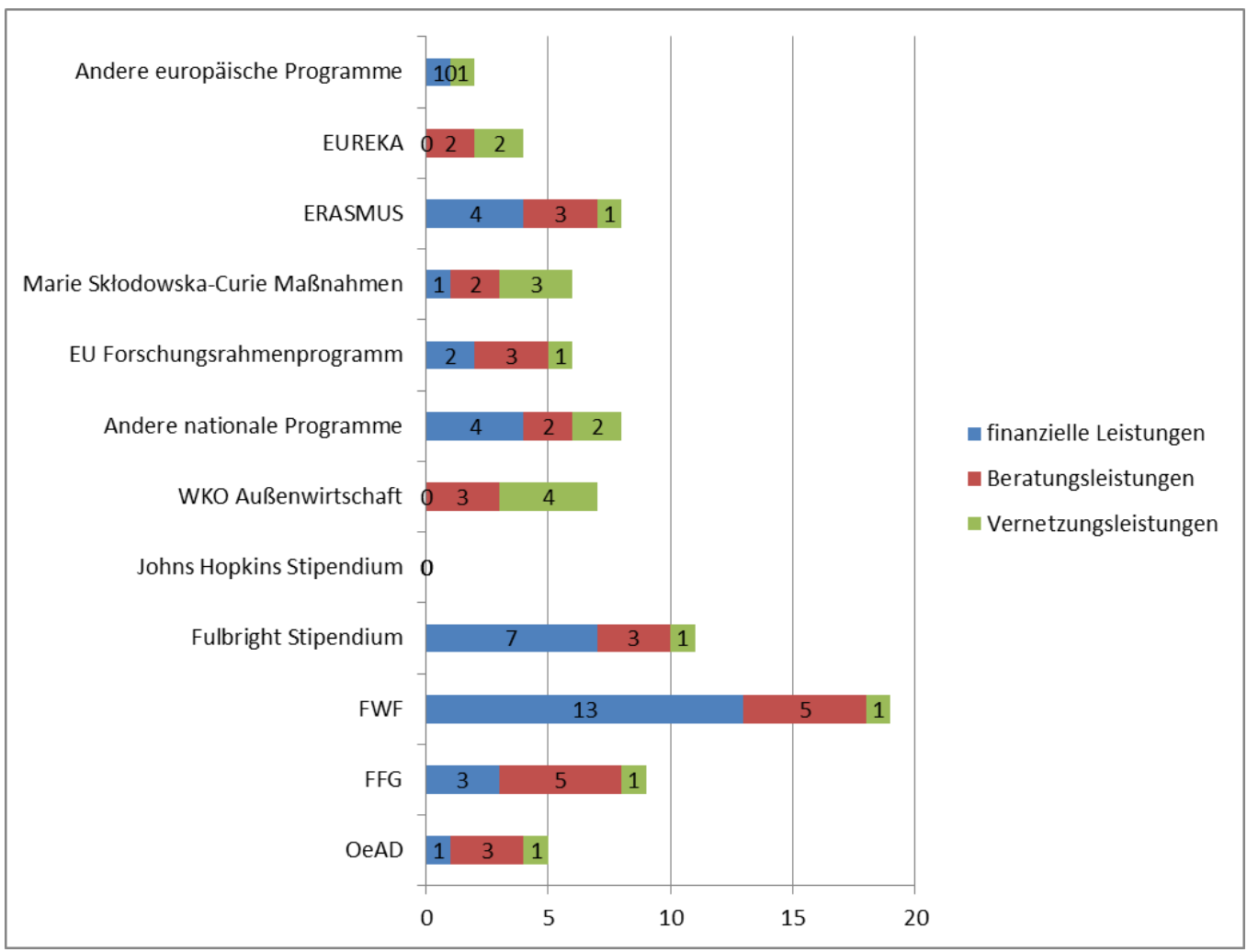

Quelle: JOANNEUM RESEARCH 


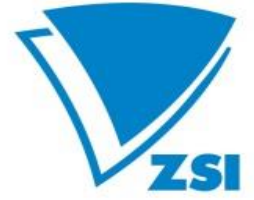

ZENTRUM FÜR SOZIALE INNOVATION

CENTRE FOR SOCIAL INNOVATION

Abbildung 28: In welchen der folgenden Bereiche sollte das OSTA Washington in Zukunft seine Aktivitäten verstärken?; $\mathrm{N}=55$

Betreuung und Beratung österreichischer Forschender in den USA

Unterstützung internationaler Mobilität (Austausch) von Forscherlnnen

Vermittlung von Jobangeboten in Österreich

Unterstützung bi- und multilateraler Kooperationen (z.B. in EU-Programmen)

Anbahnung bilateraler Kooperation zwischen Forschungseinrichtungen und Unternehmen Identifikation von potentiellen Partnern und Herstellen von Kontakten

Vermittlung von Jobangeboten in den USA

Vermarktung österreichischer Technologien in den USA

Betreuung und Beratung österreichischer Unternehmen in den USA

Unterstützung des Ausbaus bilateraler Kooperationen auf (FTI-)politischer Ebene

Informationsbereitstellung, Analysen und Daten zu den USA

Betreuung und Beratung amerikanischer Forschender in Österreich

Unterstützung bzw. (Mit-)Organisation von Delegationen

Informationsbereitstellung, Analysen und Daten zum FTIStandort Österreich

Maßnahmen zur (FTI-)Politikunterstützung

Andere

Betreuung und Beratung amerikanischer Unternehmen in Österreich

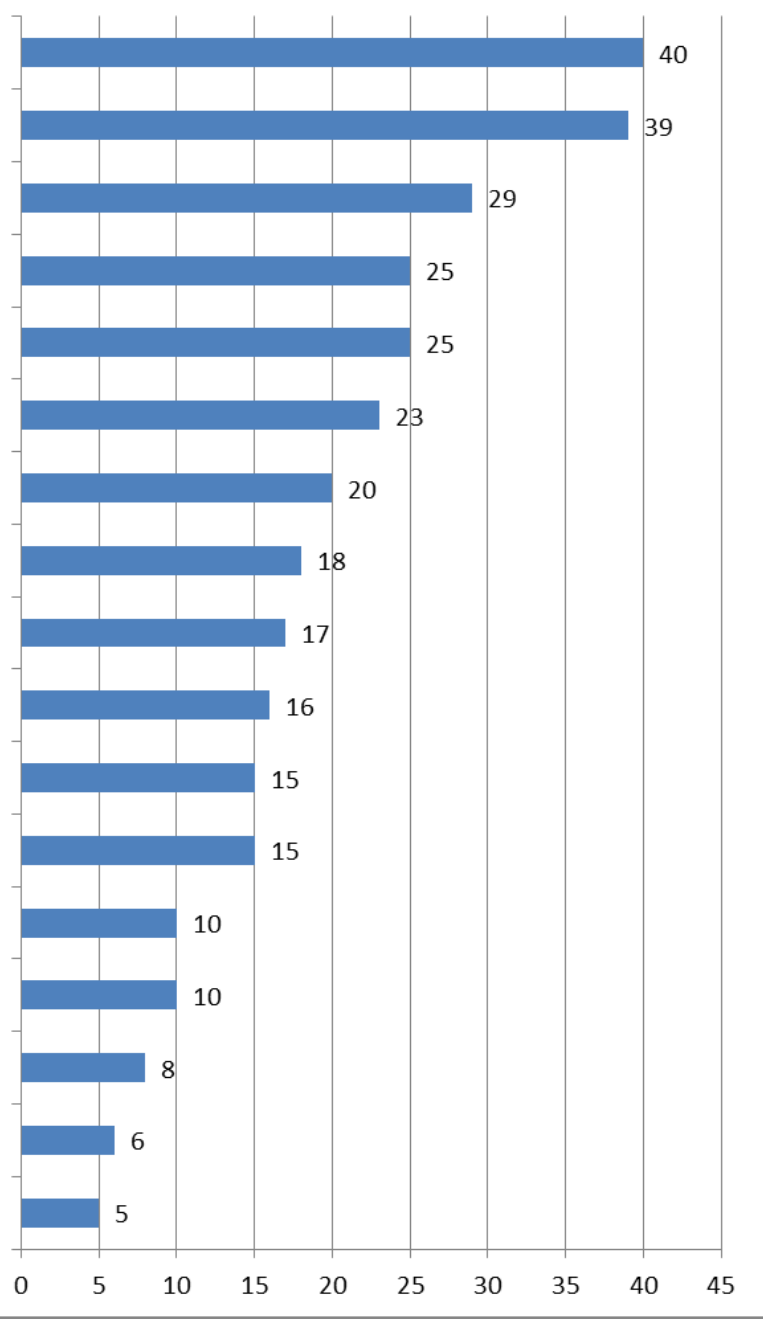

Quelle: JOANNEUM RESEARCH 


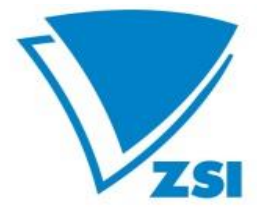

ZENTRUM FÜR SOZIALE INNOVATION

CENTRE FOR SOCIAL ININOVATION

\subsection{Ergebnisse Online Survey - Peking}

Abbildung 29: Aktivtäten von Hochschulen und Forschungseinrichtungen in China; $n=11$

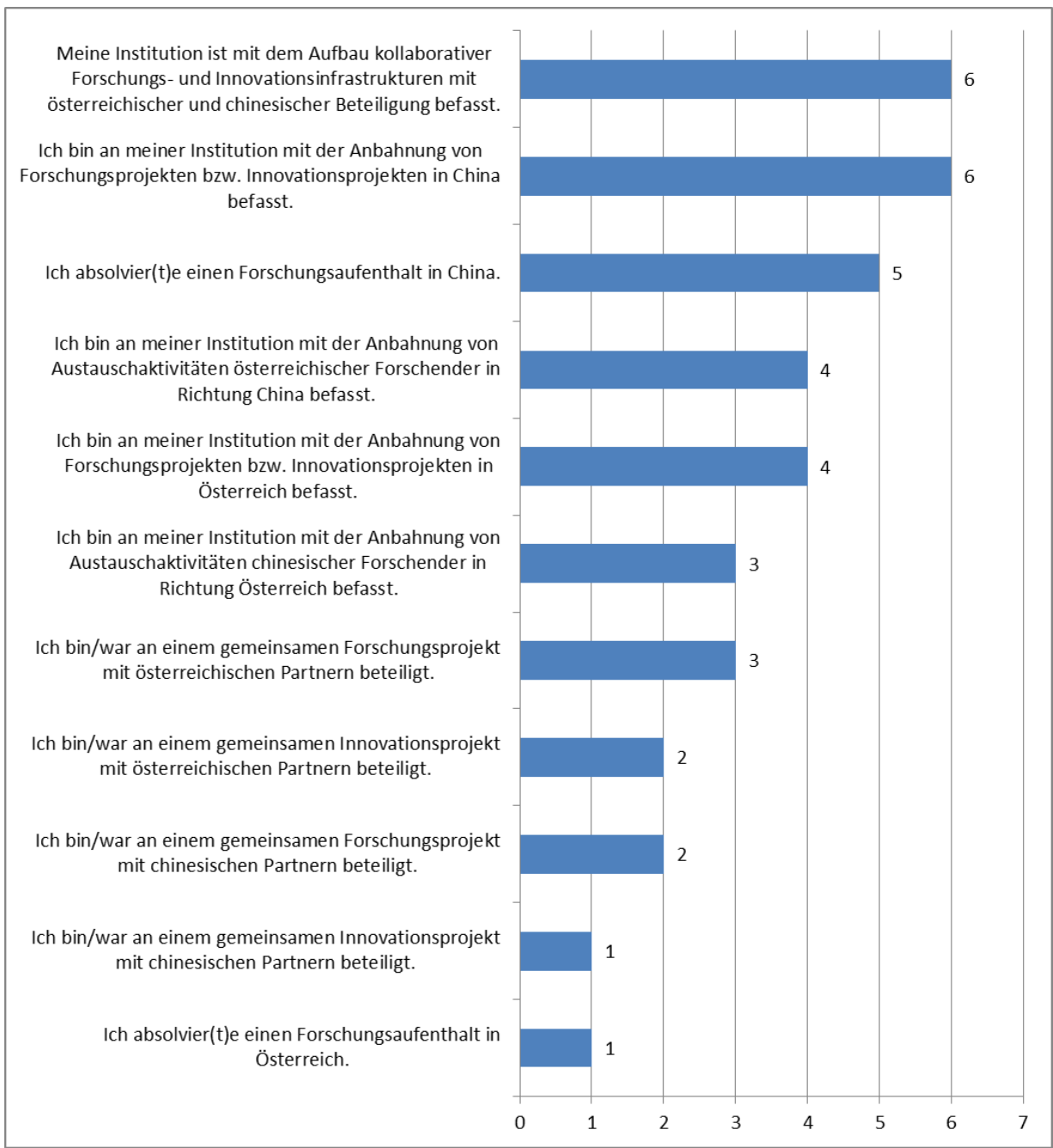

Quelle: JOANNEUM RESEARCH 


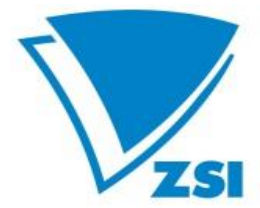

ZENTRUM FÜR SOZIALE INNOVATION

CENTRE FOR SOCIAL INNOVATION

Abbildung 30: Aktivtäten von Unternehmen in China; $n=7$

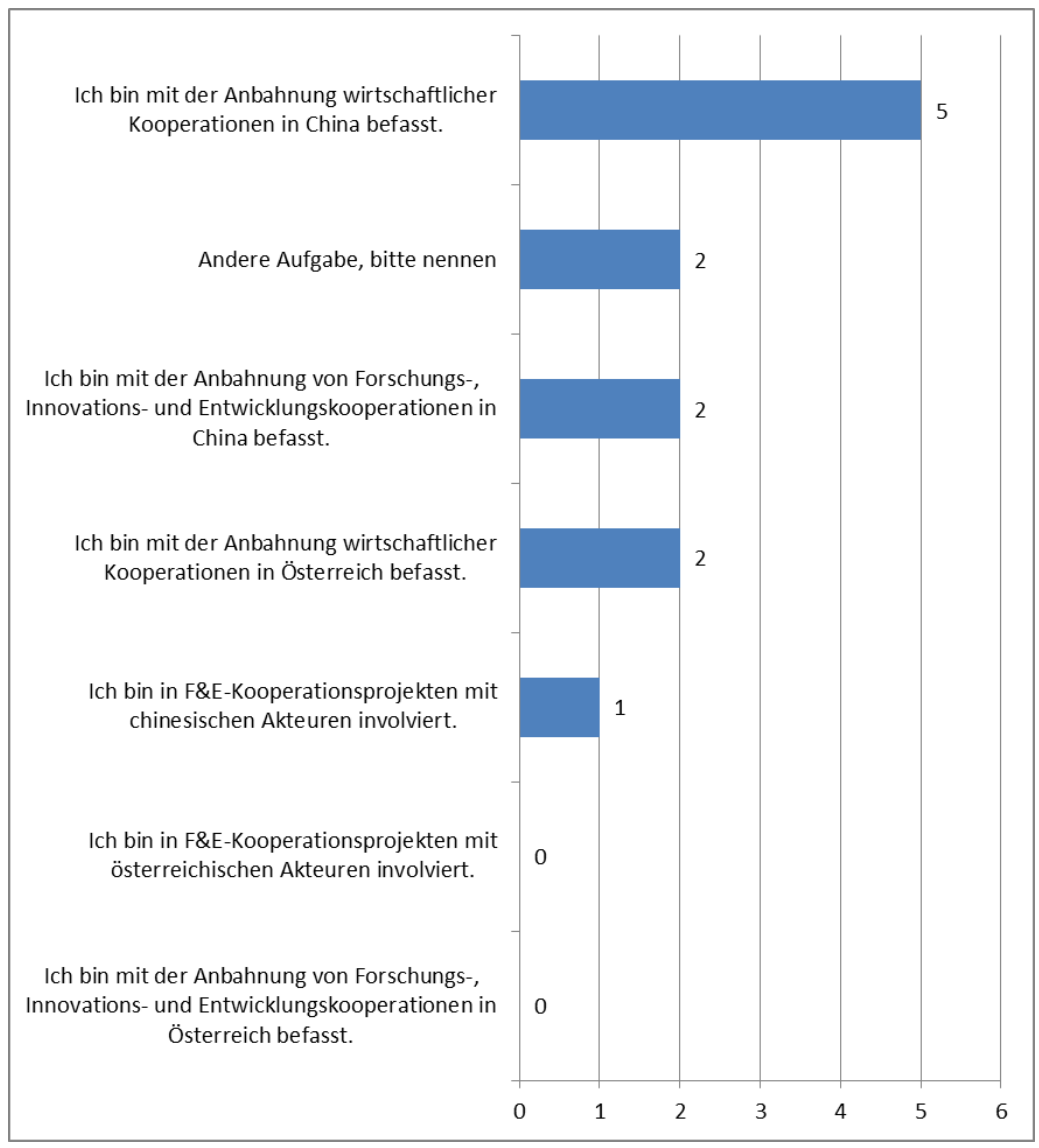

Quelle: JOANNEUM RESEARCH

Abbildung 31: Land des aktuellen Aufenthaltes; N=35

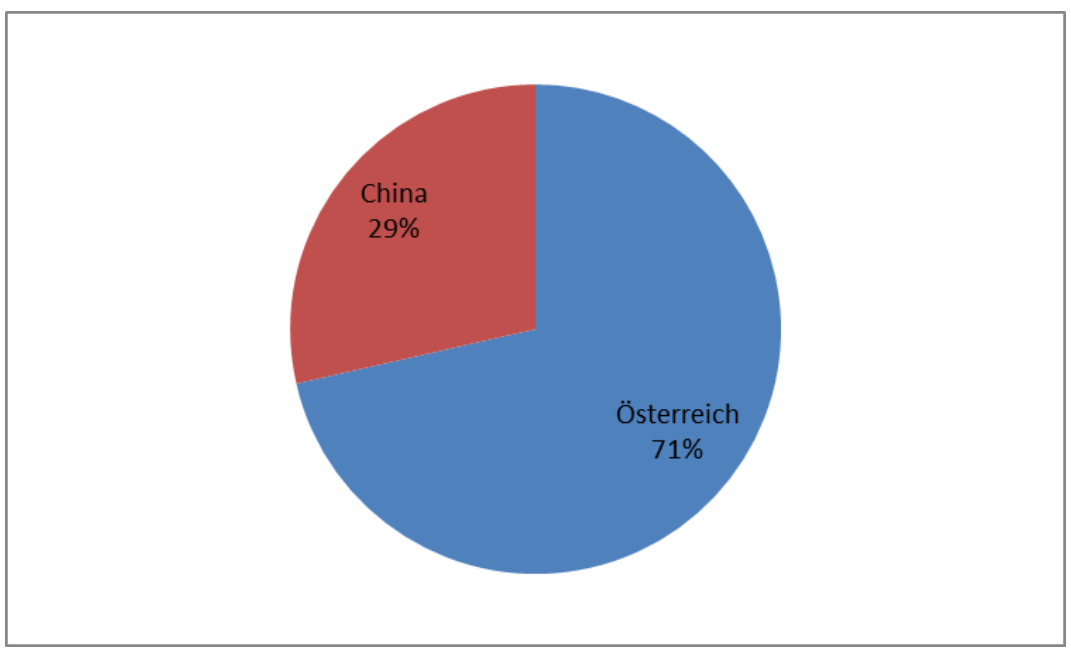

Quelle: JOANNEUM RESEARCH 


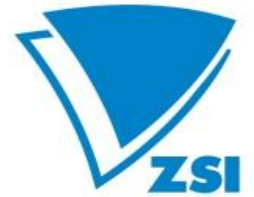

ZENTRUM FÜR SOZIALE INNOVATION

CENTRE FOR SOCIAL ININOVATION

Abbildung 32: Institution; N=35

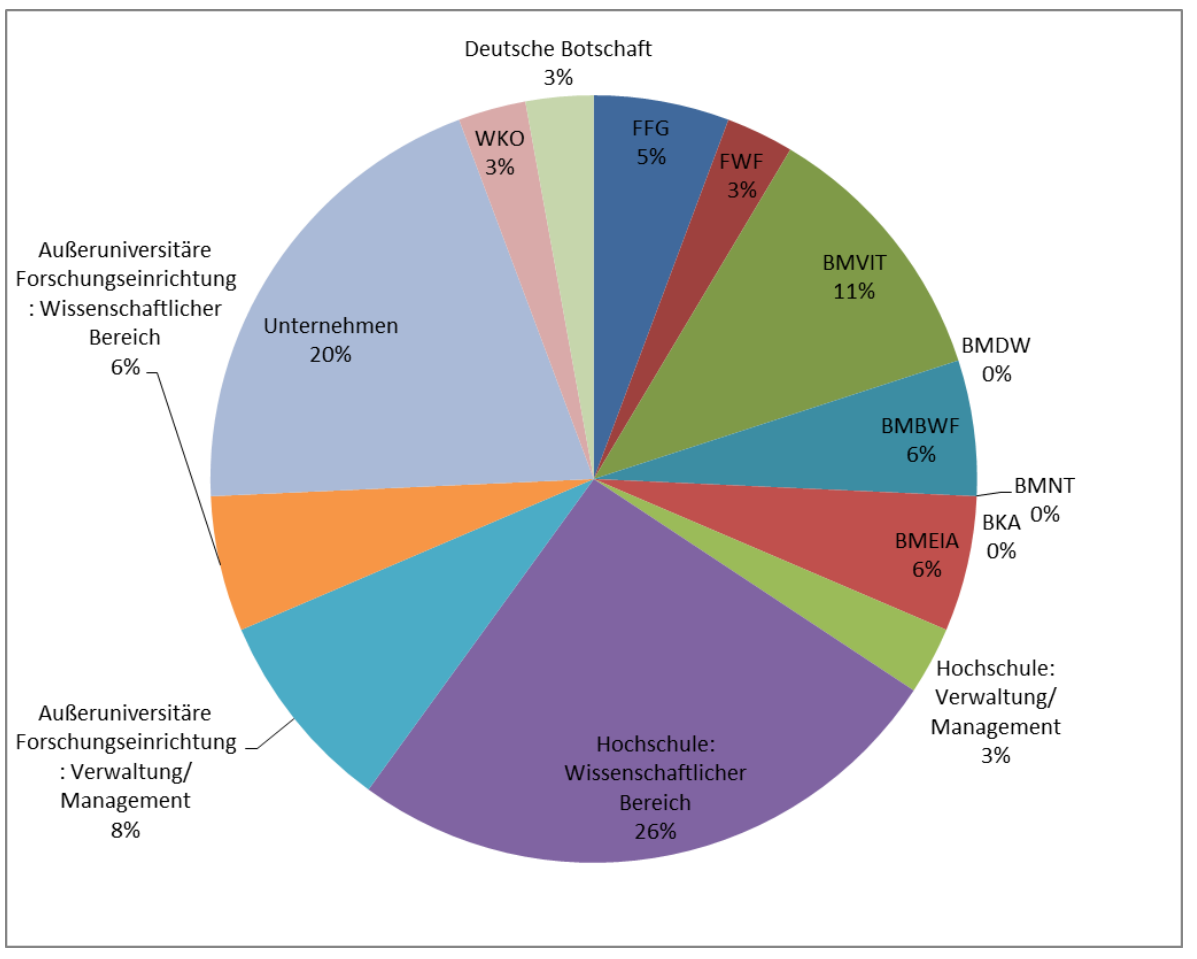

Quelle: JOANNEUM RESEARCH 


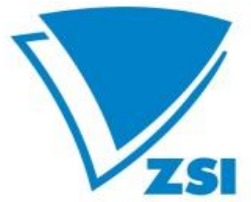

ZENTRUM FÜR SOZIALE INNOVATION

CENTRE FOR SOCIAL ININOVATION

Abbildung 33: Wie haben Sie von der Existenz des OSTA Peking erfahren?; Wissenschaftlicher Bereich Hochschulen und Außeruniversitäre Forschung, Skala=Anzahl; N=12

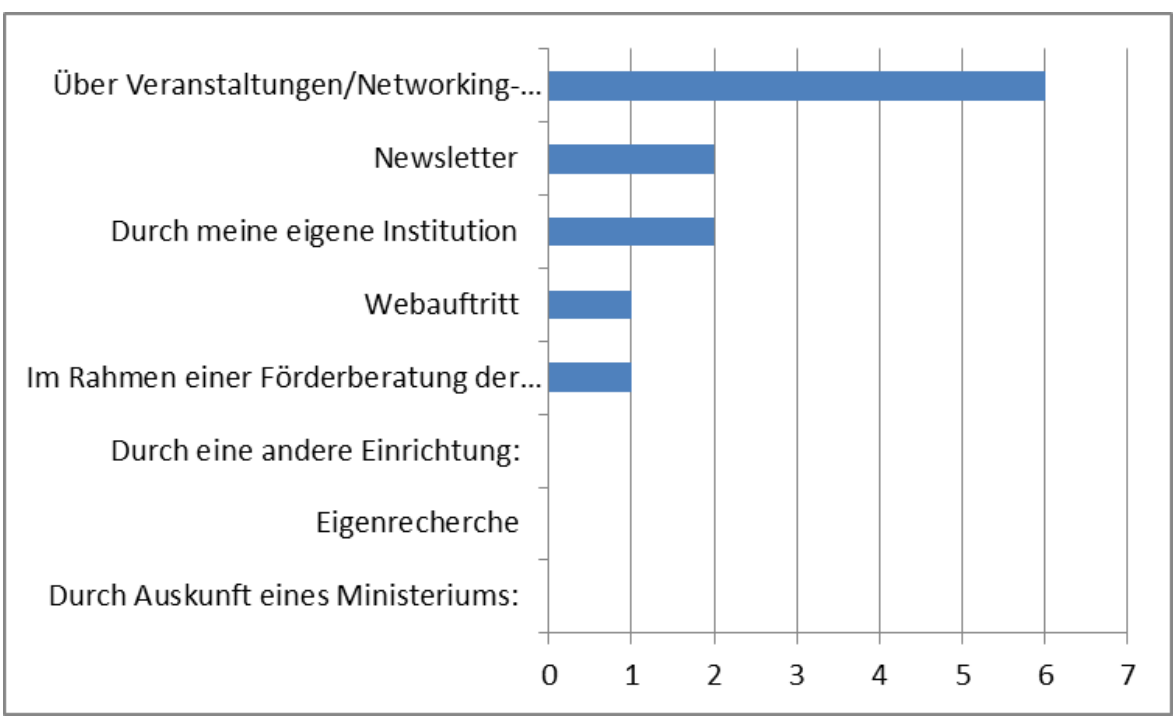

Quelle: JOANNEUM RESEARCH

Drei Unternehmen haben diese Frage beantwortet, dabei wurden jeweils einmal die Förderberatung der FFG, das eigene Unternehmen sowie eine Veranstaltung als Quelle angegeben. 


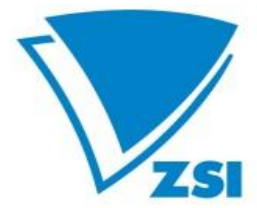

ZENTRUM FÜR SOZIALE INNOVATION

CENTRE FOR SOCIAL INNOVATION

Abbildung 34: Bitte beurteilen Sie die Relevanz folgender Aktivitätsbereiche des OSTA Peking aus Ihrer Perspektive;

Skala=Anzahl; N=Itemabhängig max. 25

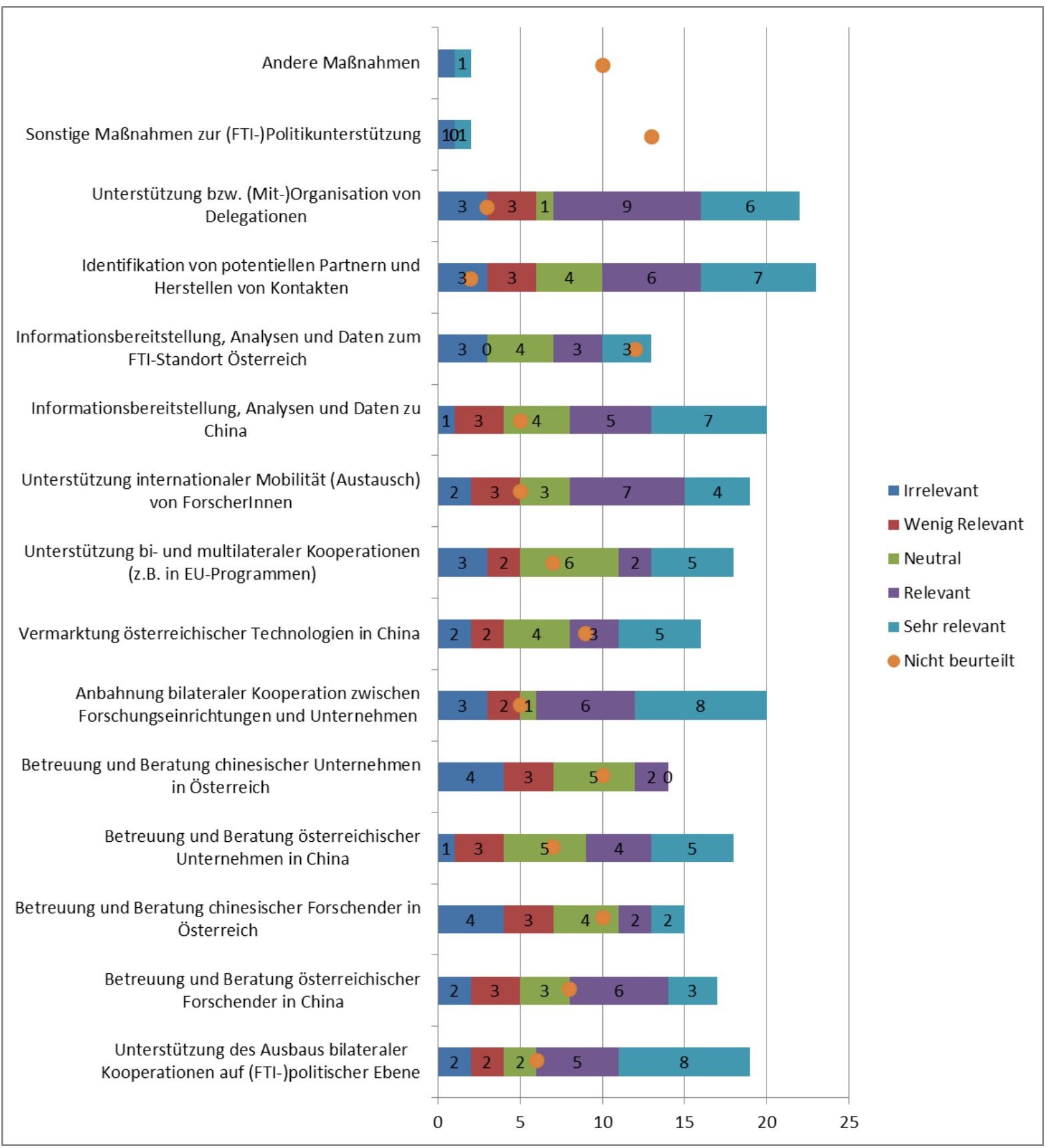

Quelle: JOANNEUM RESEARCH 


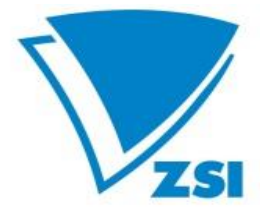

ZENTRUM FÜR SOZIALE INNOVATION

CENTRE FOR SOCIAL INNOVATION

Abbildung 35: Wie zufrieden sind/waren Sie mit den entsprechenden Leistungen?; Skala=Anzahl; N=Itemabhängig max.

19

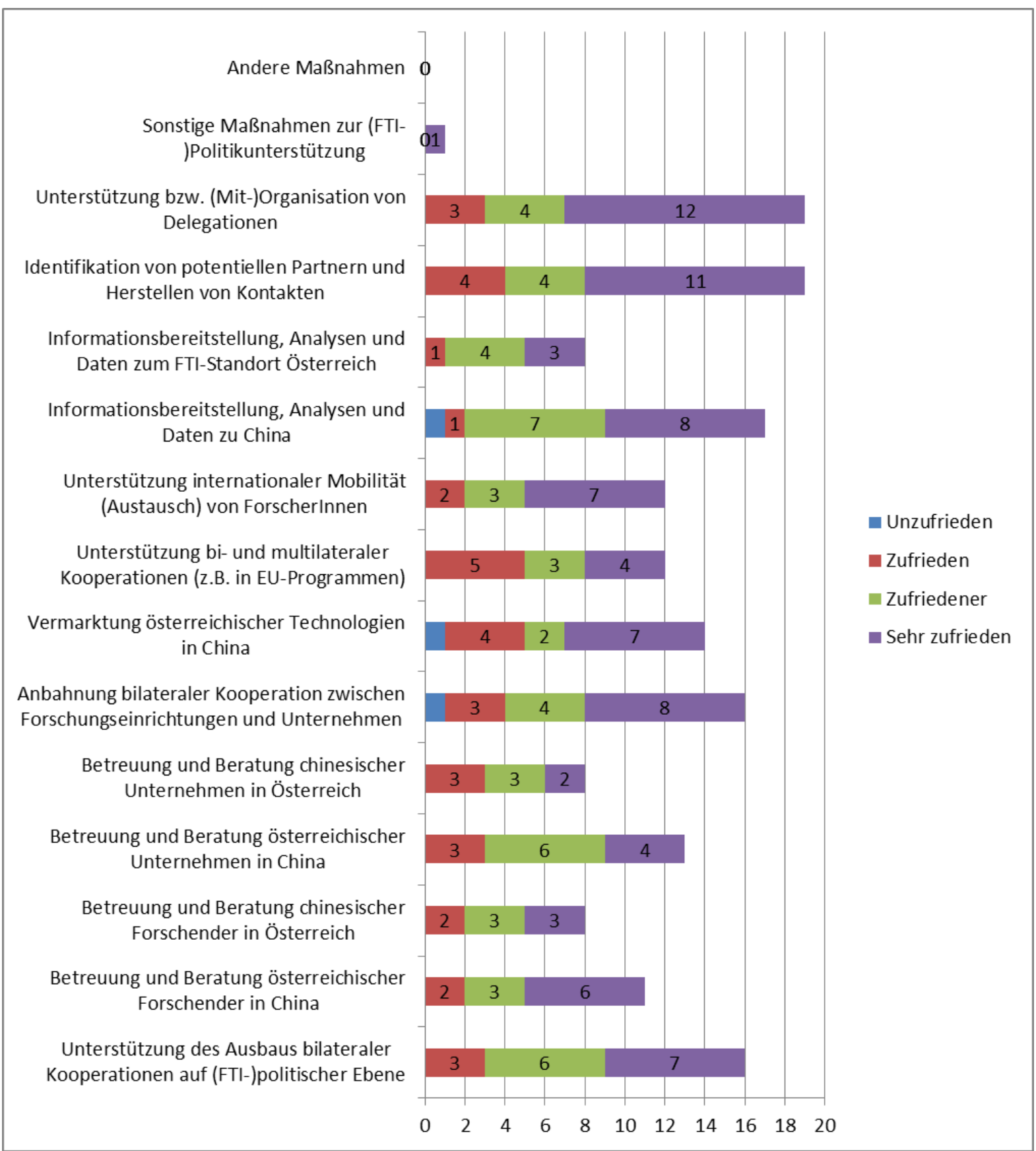

Quelle: JOANNEUM RESEARCH 


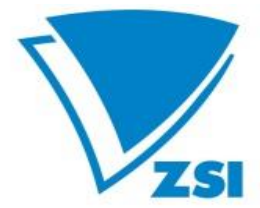

ZENTRUM FÜR SOZIALE INNOVATION

CENTRE FOR SOCIAL ININOVATION

Abbildung 36: Bitte beurteilen Sie die Relevanz folgender Aktivitätsbereiche des OSTA Peking aus Ihrer Perspektive;

Wissenschaftlicher Bereich Hochschulen und Außeruniversitäre Forschung; Skala=Anzahl; N=12

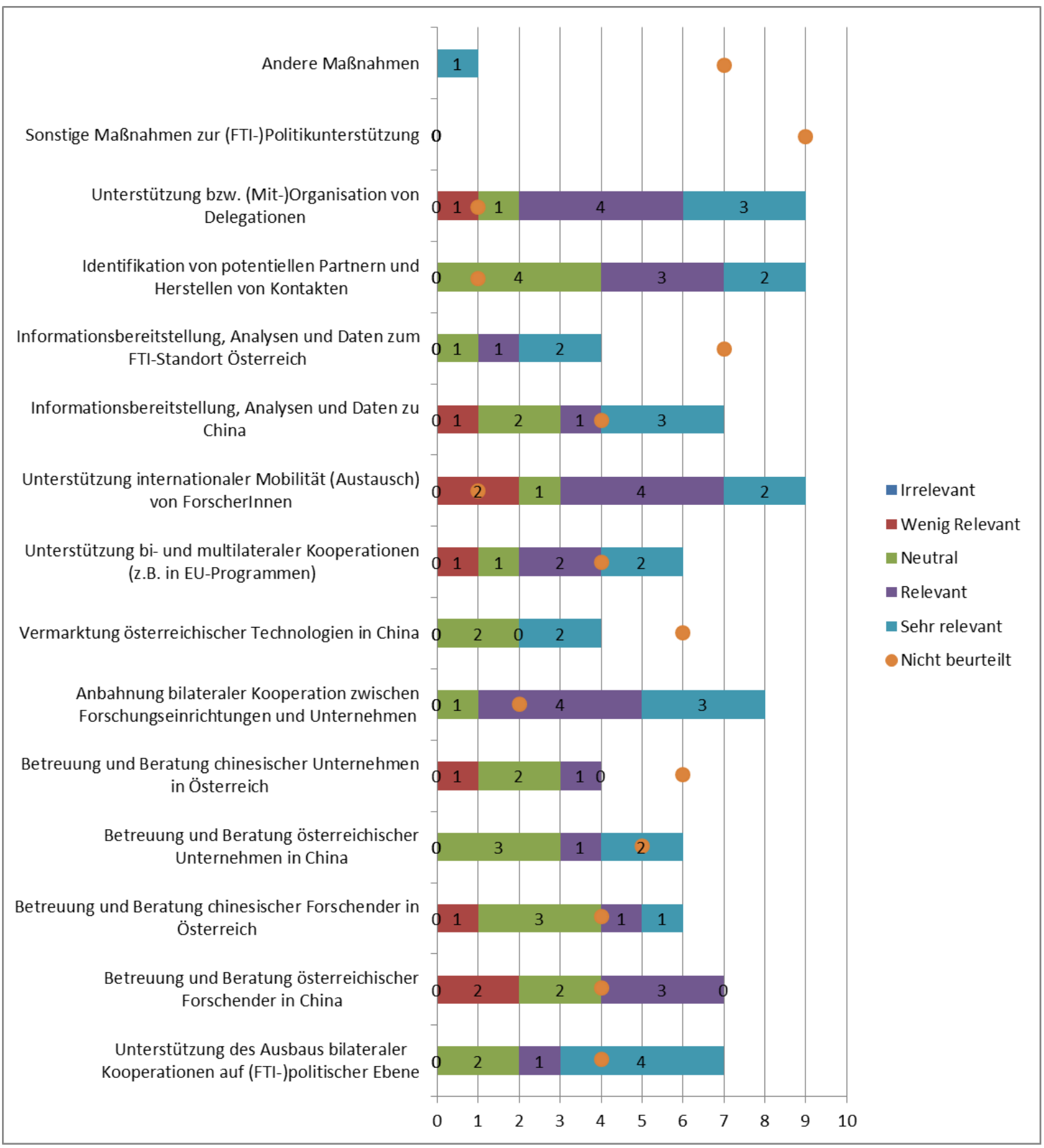

Quelle: JOANNEUM RESEARCH 


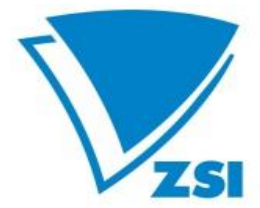

ZENTRUM FÜR SOZIALE INNOVATION

CENTRE FOR SOCIAL INNOVATION

Abbildung 37: Wie zufrieden sind/waren Sie mit den entsprechenden Leistungen?; Wissenschaftlicher Bereich

Hochschulen und Außeruniversitäre Forschung; Skala=Anzahl; N=Itemabhängig max. 9

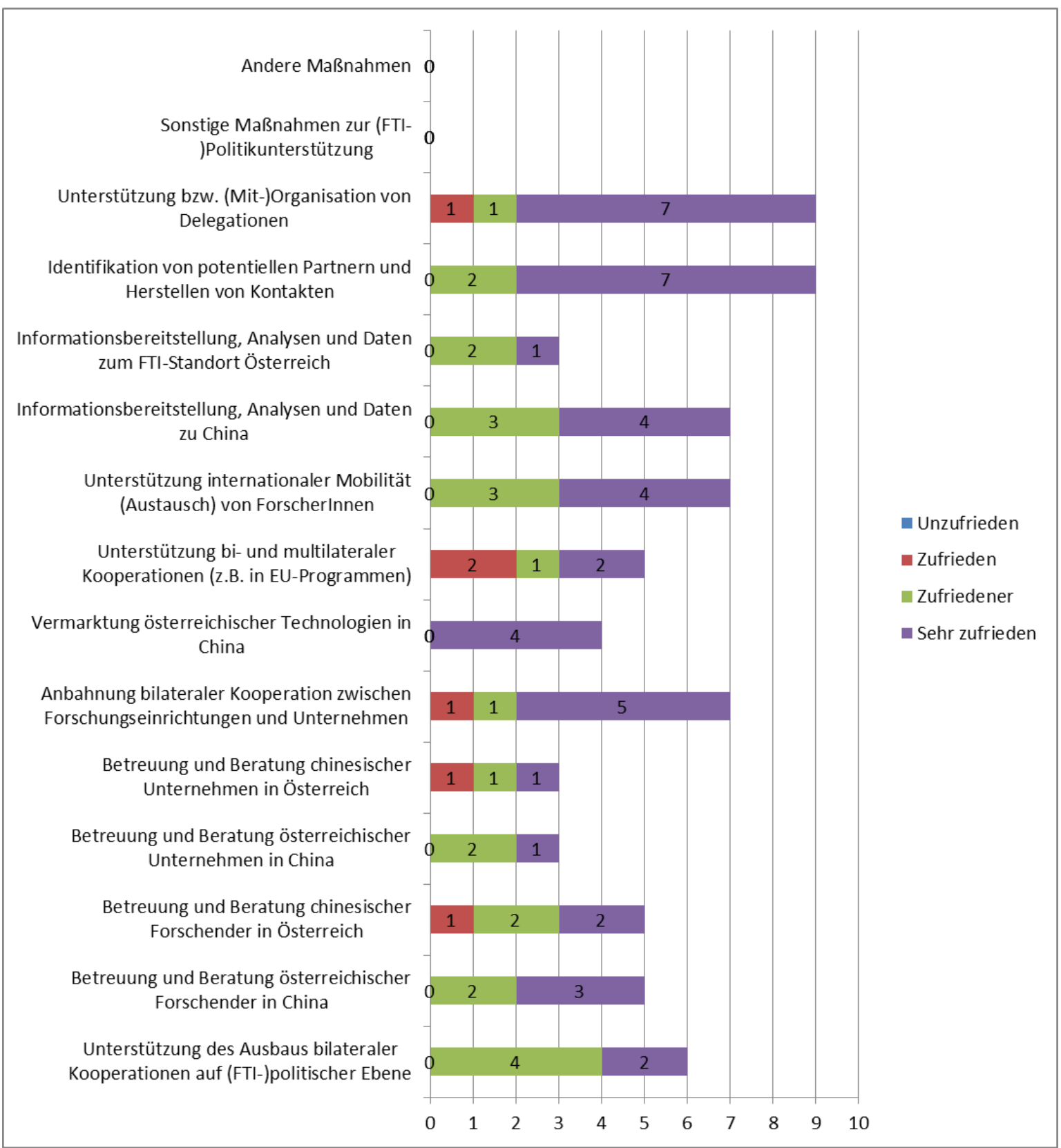

Quelle: JOANNEUM RESEARCH 


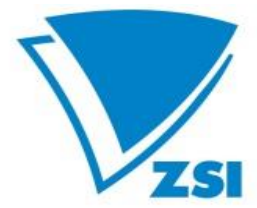

ZENTRUM FÜR SOZIALE INNOVATION

CENTRE FOR SOCIAL INNOVATION

Abbildung 38: Welche der folgenden konkreten Leistungen des OSTA Peking haben Sie in Anspruch genommen?;

$\mathrm{N}=$ Itemabhängig max. 24

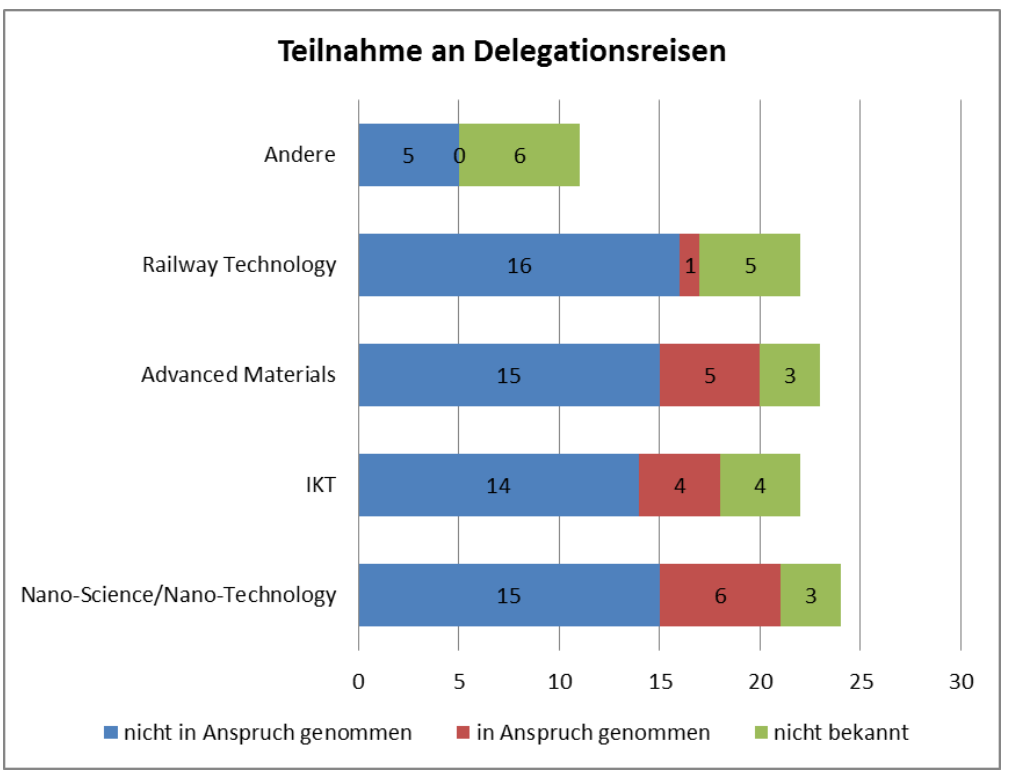

Quelle: JOANNEUM RESEARCH

Abbildung 39: Welche der folgenden konkreten Leistungen des OSTA Peking haben Sie in Anspruch genommen?;

N=Itemabhängig max. 24

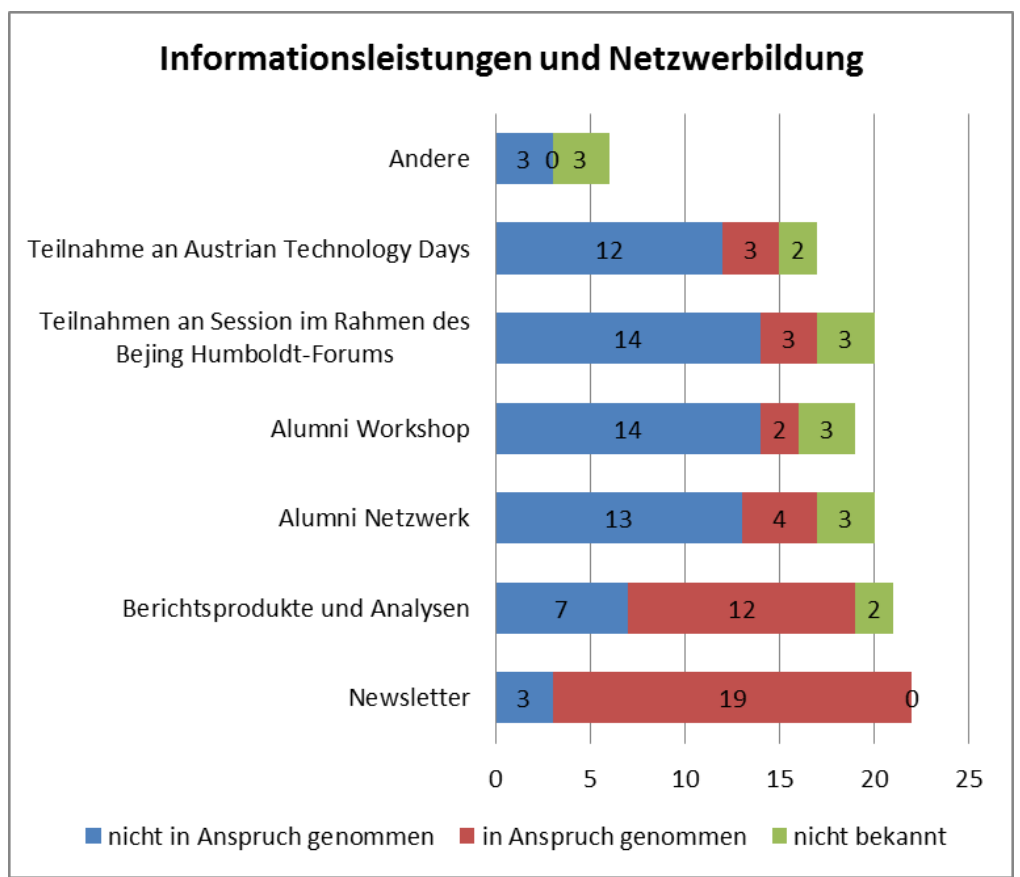

Quelle: JOANNEUM RESEARCH 


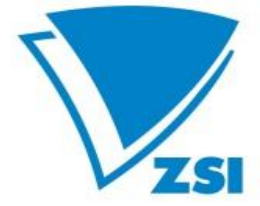

ZENTRUM FÜR SOZIALE INNOVATION

CENTRE FOR SOCIAL INNOVATION

Abbildung 40: Welche der folgenden konkreten Leistungen des OSTA Peking haben Sie in Anspruch genommen?;

$\mathrm{N}=$ Itemabhängig max. 24

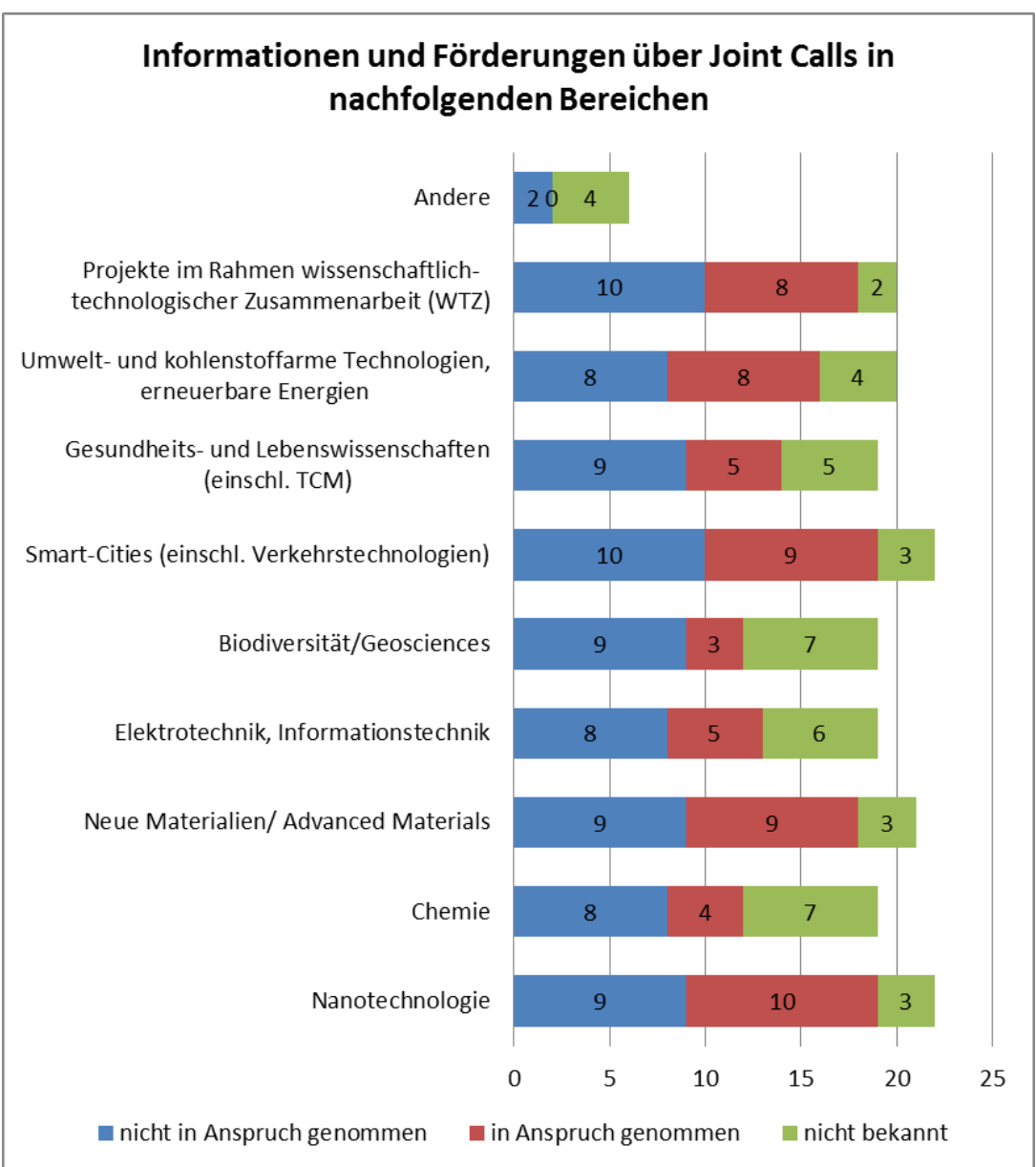

Quelle: JOANNEUM RESEARCH 


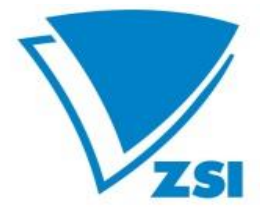

ZENTRUM FÜR SOZIALE INNOVATION

CENTRE FOR SOCIAL ININOVATION

Abbildung 41: Wie zufrieden sind Sie mit den entsprechenden Leistungen des OSTA Peking?; Skala=Anzahl; $\mathrm{N}=$ Itemabhängig max. 10

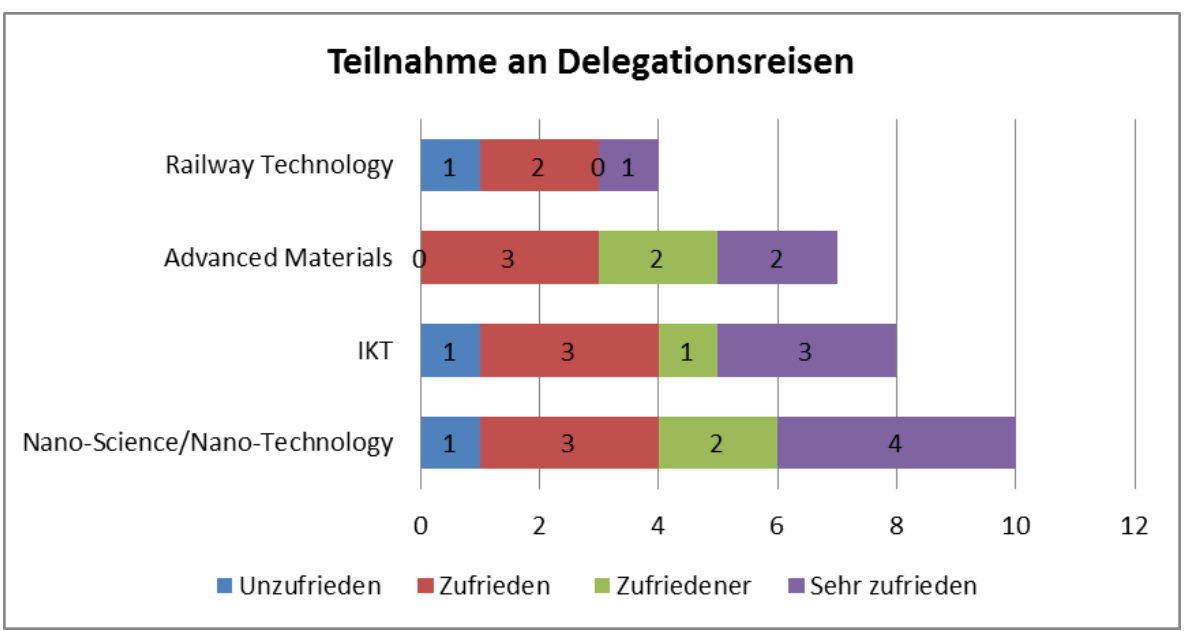

Quelle: JOANNEUM RESEARCH

Abbildung 42: Wie zufrieden sind Sie mit den entsprechenden Leistungen des OSTA Peking?; Skala=Anzahl; $\mathbf{N}=$ Itemabhängig max. 10

\section{Informationen und Förderungen über Joint Calls in nachfolgenden Bereichen}

Projekte im Rahmen wissenschaftlich-technologischer Zusammenarbeit (WTZ)

Umwelt- und kohlenstoffarme Technologien, erneuerbare Energien

Gesundheits- und Lebenswissenschaften (einschl. TCM)

Smart-Cities (einschl. Verkehrstechnologien)

Biodiversität/Geosciences

Elektrotechnik, Informationstechnik

Neue Materialien/ Advanced Materials
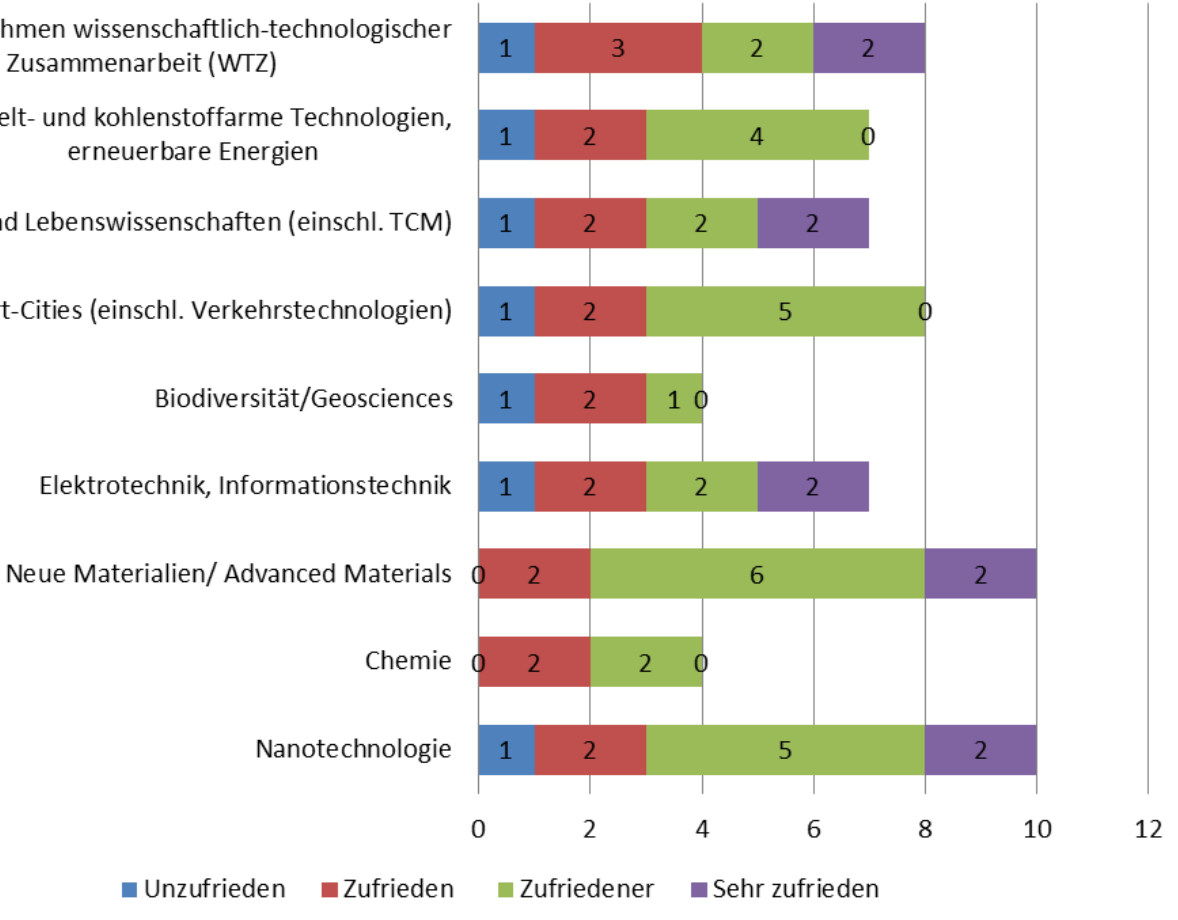


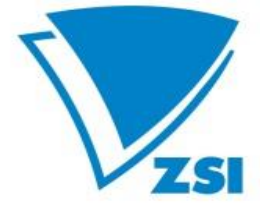

ZENTRUM FÜR SOZIALE INNOVATION

CENTRE FOR SOCIAL ININOVATION

Quelle: JOANNEUM RESEARCH

Abbildung 43: Wie zufrieden sind Sie mit den entsprechenden Leistungen des OSTA Peking?; Skala=Anzahl;

$\mathrm{N}=$ Itemabhängig max. 18

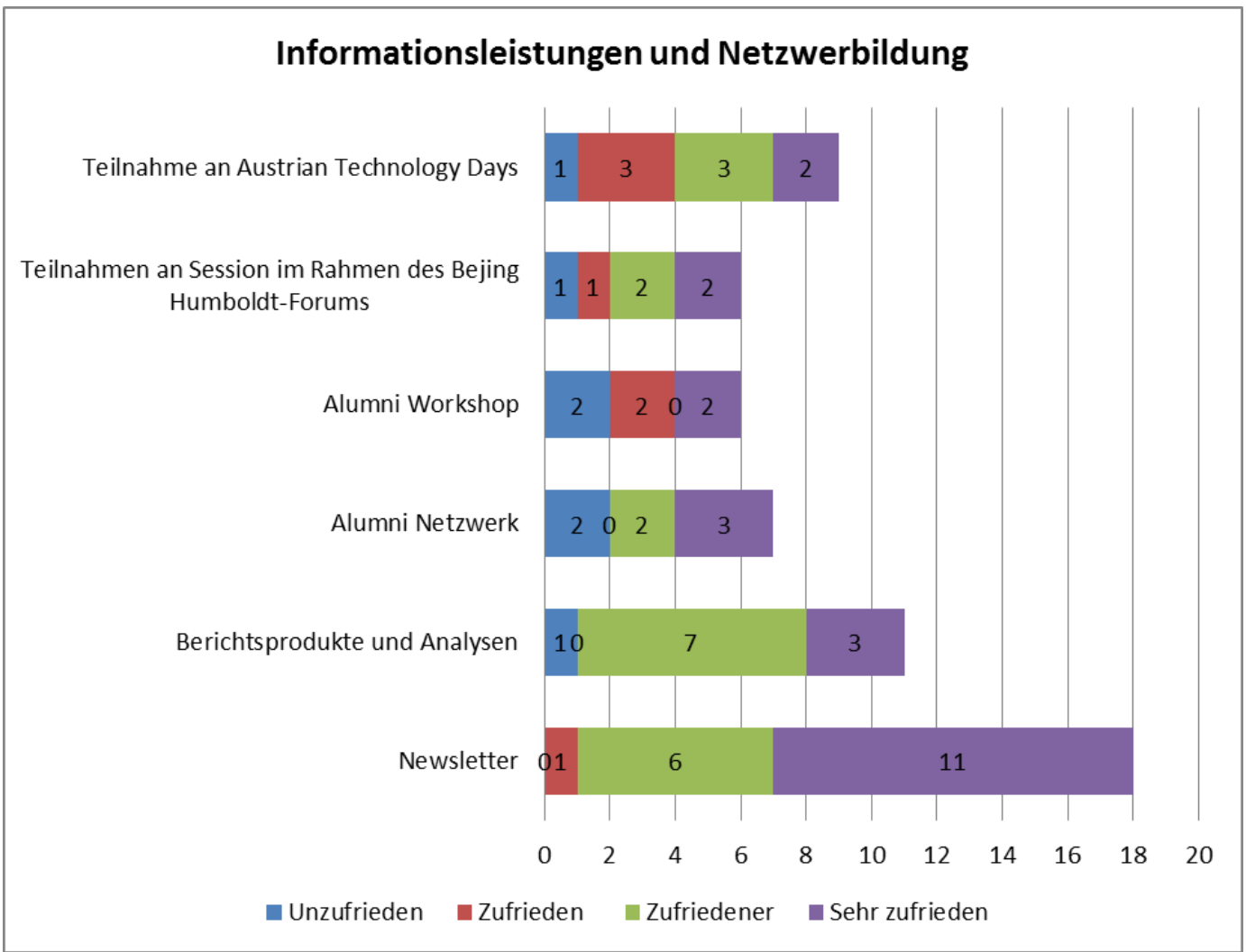

Quelle: JOANNEUM RESEARCH 


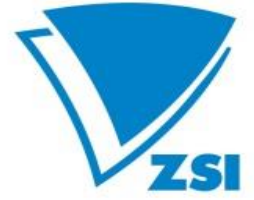

ZENTRUM FÜR SOZIALE INNOVATION

CENTRE FOR SOCIAL ININOVATION

Abbildung 44: War OSTA Peking die wichtigste Unterstützung/Informationsquelle, die Sie als Grundlage für Ihre

Aktivitäten herangezogen haben?; $\mathrm{N}=17$

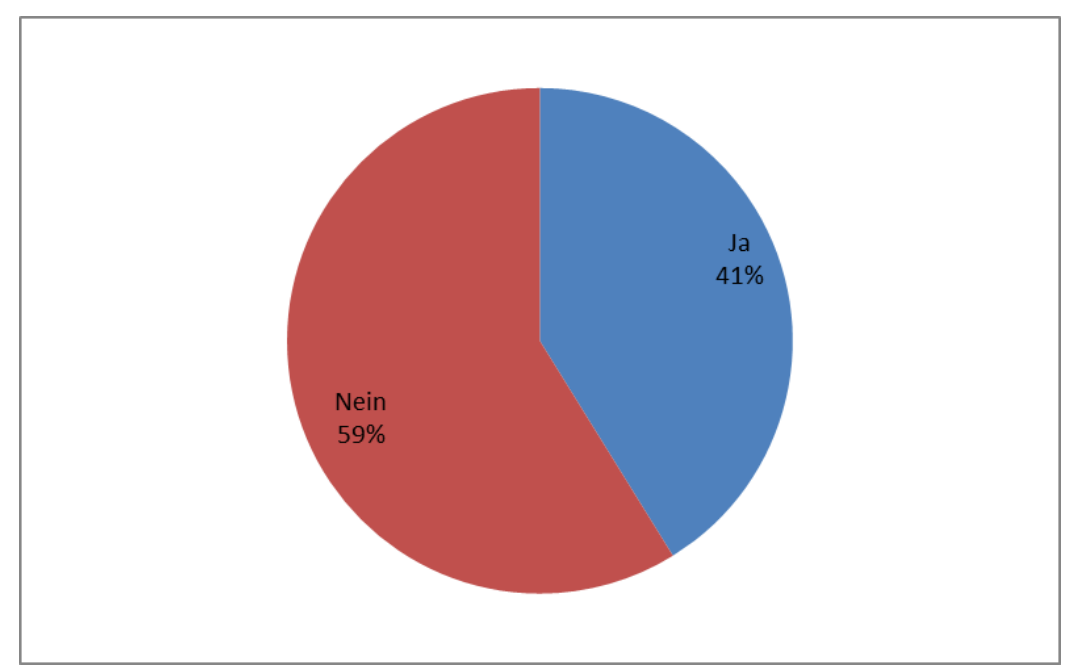

Quelle: JOANNEUM RESEARCH 


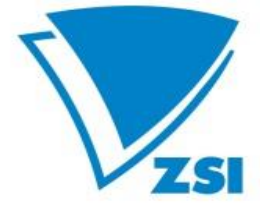

ZENTRUM FÜR SOZIALE INNOVATION

CENTRE FOR SOCIAL INNOVATION

Abbildung 45: Auf welche anderen Unterstützungsleistungen, Programme oder Akteure haben Sie zurückgegriffen?;

Skala=Anzahl; $\mathbf{N}=10$

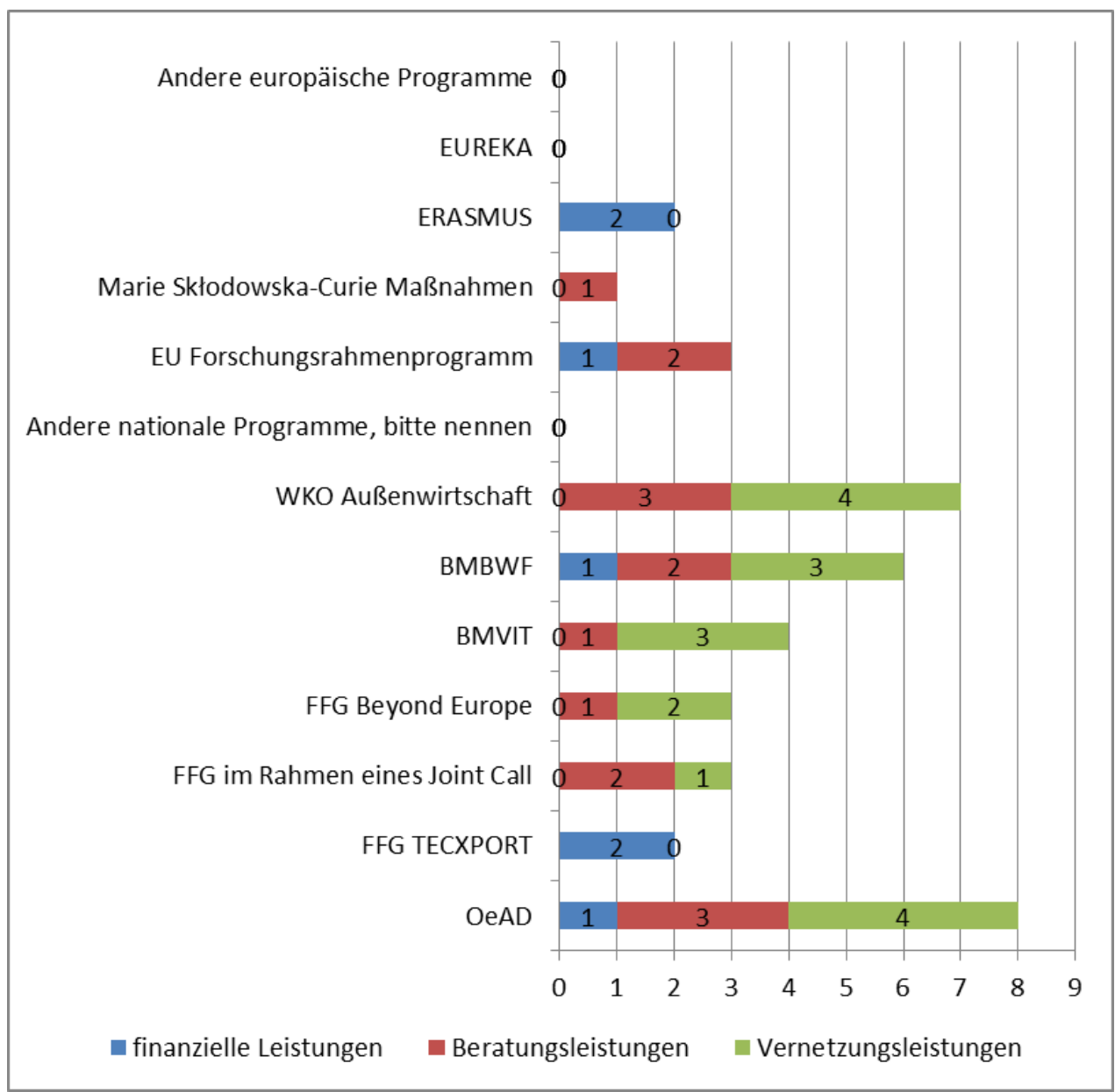

Quelle: JOANNEUM RESEARCH 


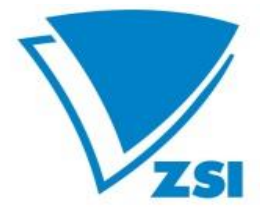

ZENTRUM FÜR SOZIALE INNOVATION

CENTRE FOR SOCIAL ININOVATION

Abbildung 45: In welchen der folgenden Bereiche sollte das OSTA Washington in Zukunft seine Aktivitäten verstärken?; $\mathrm{N}=\mathbf{2 6}$

Informationsbereitstellung, Analysen und Daten zu China

Identifikation von potentiellen Partnern und Herstellen von Kontakten

Anbahnung bilateraler Kooperation zwischen Forschungseinrichtungen und Unternehmen Unterstützung internationaler Mobilität (Austausch) von Forscherlnnen

Unterstützung bi- und multilateraler Kooperationen (z.B. in EU-Programmen)

Unterstützung des Ausbaus bilateraler Kooperationen auf (FTI-)politischer Ebene

Informationsbereitstellung, Analysen und Daten zum FTI-Standort Österreich

Vermarktung österreichischer Technologien in China

Betreuung und Beratung österreichischer Forschender in China

Unterstützung bzw. (Mit-)Organisation von Delegationen

Maßnahmen zur (FTI-)Politikunterstützung

Vermittlung von Jobangeboten in China

Betreuung und Beratung österreichischer Unternehmen in China

Betreuung und Beratung chinesischer Forschender in Österreich

Vermittlung von Jobangeboten in Österreich

Andere Aktivität

Betreuung und Beratung chinesischer Unternehmen in Österreich

In welchen der folgenden Bereiche sollte das OSTA Peking in Zukunft seine Aktivitäten verstärken?

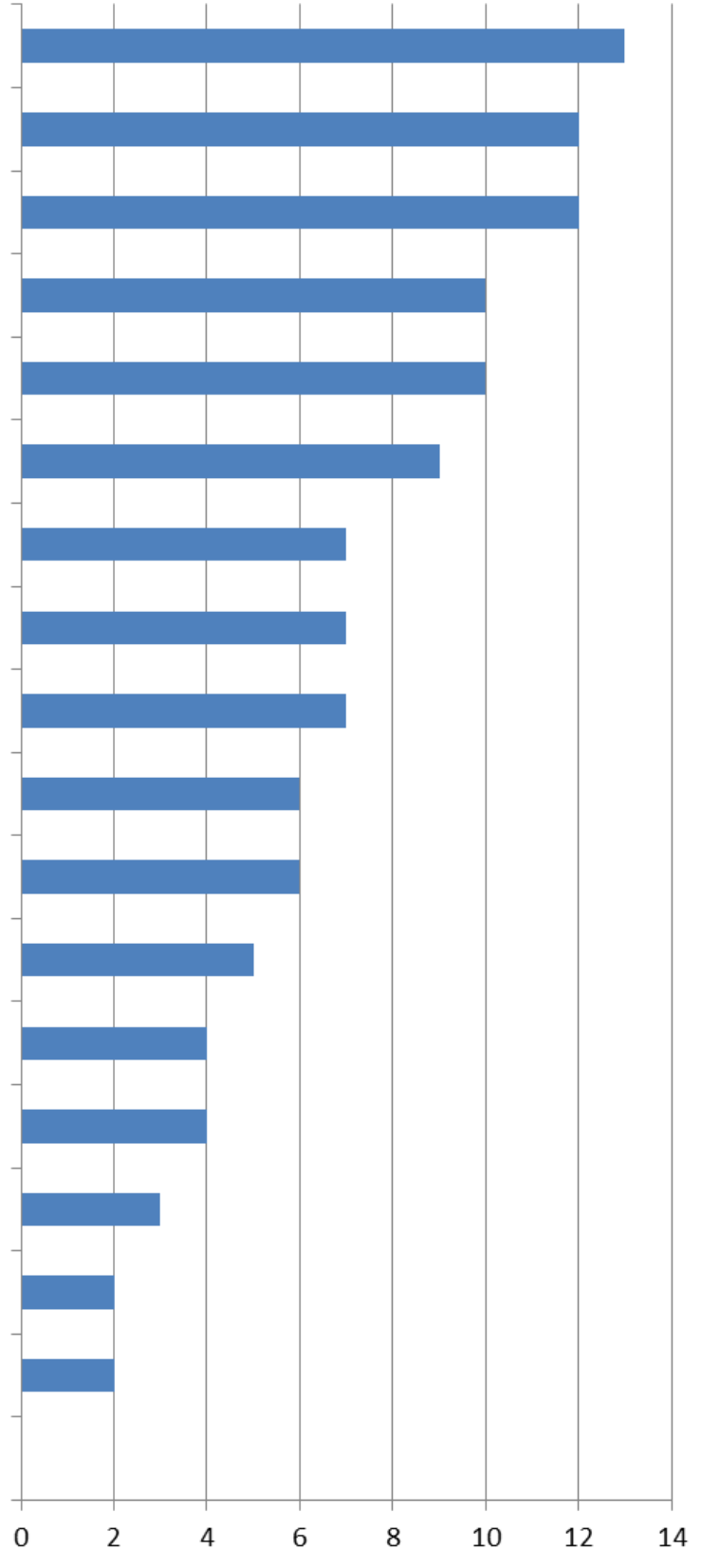

Quelle: JOANNEUM RESEARCH 


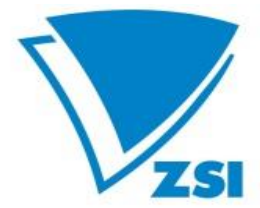

ZENTRUM FÜR SOZIALE INNOVATION

CENTRE FOR SOCIAL INNOVATION

Abbildung 46: In welchen der folgenden Bereiche sollte das OSTA Peking in Zukunft seine Aktivitäten verstärken?;

Wissenschaftlicher Bereich Hochschulen und Außeruniversitäre Forschung; N=9

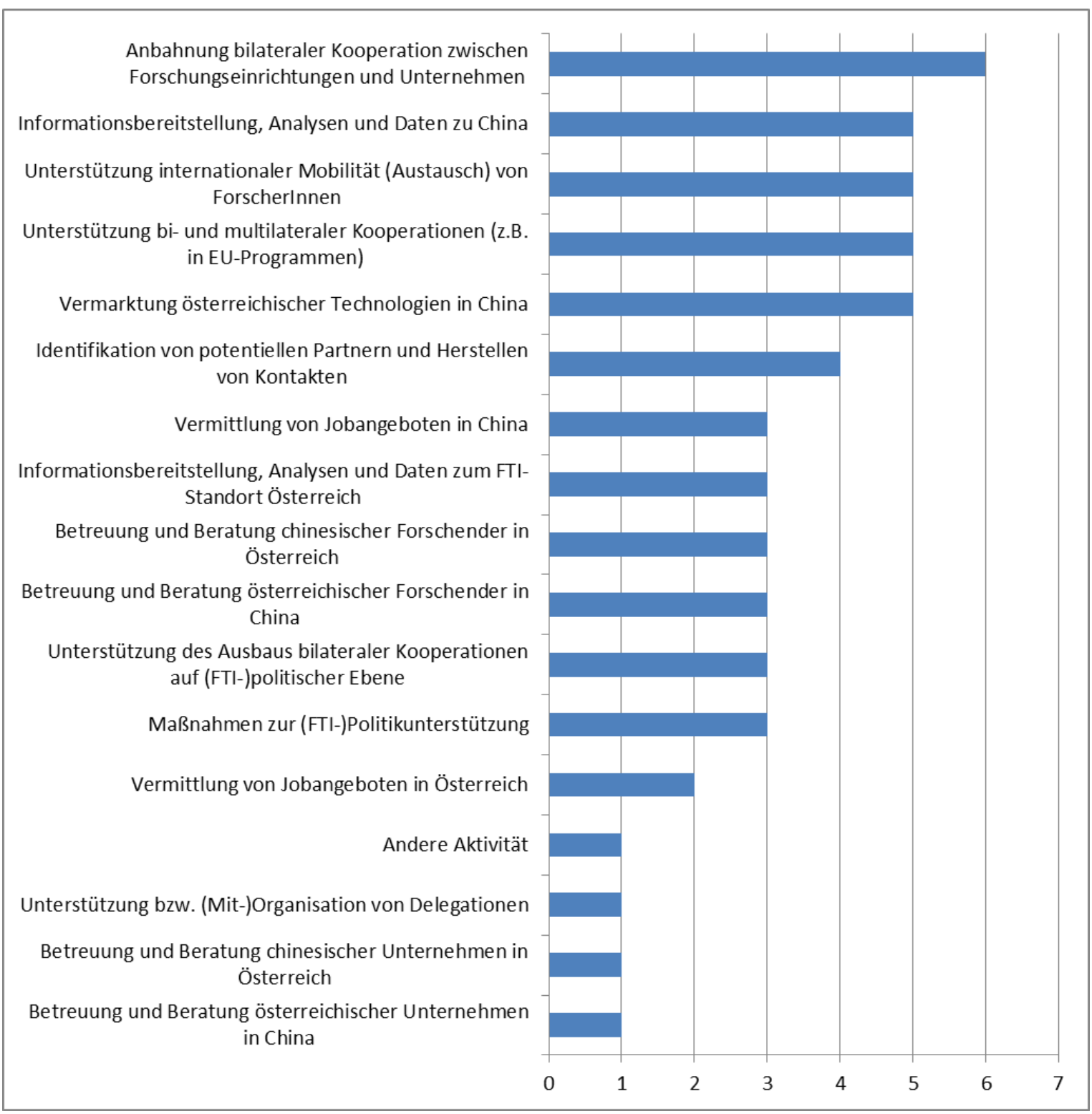

Quelle: JOANNEUM RESEARCH 